\title{
Resonant leading order geometric optics expansions for quasilinear hyperbolic fixed and free boundary problems
}

\author{
Jean-Francois Coulombel, Olivier Gues ${ }^{\dagger}$ Mark Williams ${ }^{\ddagger}$
}

September 27, 2010

\begin{abstract}
We provide a justification with rigorous error estimates showing that the leading term in weakly nonlinear geometric optics expansions of highly oscillatory reflecting wavetrains is close to the uniquely determined exact solution for small wavelengths $(\epsilon)$. Waves reflecting off of fixed noncharacteristic boundaries and off of multidimensional shocks are considered under the assumption that the underlying fixed (respectively, free) boundary problem is uniformly spectrally stable in the sense of Kreiss (respectively, Majda). Our results apply to a general class of problems that includes the compressible Euler equations; as a corollary we rigorously justify the leading term in the geometric optics expansion of highly oscillatory multidimensional shock solutions of the Euler equations. An earlier stability result of this type [21] was obtained by a method that required the construction of high-order approximate solutions. That construction in turn was possible only under a generically valid (absence of) small divisors assumption. Here we are able to remove that assumption and avoid the need for high-order expansions by studying associated singular (because they involve coefficients of order $\frac{1}{\epsilon}$ ) fixed and free boundary problems. The analysis applies equally to systems that cannot be written in conservative form.
\end{abstract}

\section{Contents}

1 Introduction $\quad 2$

1.1 Exact solutions and singular systems. . . . . . . . . . . . . . 3

1.2 Assumptions and main results. . . . . . . . . . . . . . 6

1.3 From almost periodic profiles to mode-by-mode analysis. . . . . . . . . . . . 12

1.4 Error analysis by simultaneous Picard iteration . . . . . . . . . . . . 18

${ }^{*}$ CNRS and Université Lille 1, Laboratoire Paul Painlevé, Bâtiment M2, Cité scientifique, 59655 Villeneuve d'Ascq Cedex, France; Research of the author was supported by the French Agence Nationale de la Recherche, contract ANR-08-JCJC-0132-01.

${ }^{\dagger}$ LATP, Université de Provence; gues@cmi.univ-mrs.fr.; Research of O.G. was partially supported by the French Agence Nationale de la Recherche, contract ANR-08-JCJC-0132-01.

‡University of North Carolina; williams@email.unc.edu.; Research of M.W. was partially supported by NSF grants number DMS-0701201 and DMS-1001616. 
2.1 Profile equations: formulation with almost-periodic profiles . . . . . . . 20

2.2 Profile equations: reformulation with periodic profiles . . . . . . . . . . 22

2.3 The large system for individual modes. . . . . . . . . . . . . . . . . 26

2.4 Solution of the large system. . . . . . . . . . . . . . . . . . . 32

2.5 Error analysis . . . . . . . . . . . . . . . . . 38

3 A free boundary problem: highly oscillatory shocks 43

3.1 Assumptions. . . . . . . . . . . . . . . . . . 44

3.2 The singular shock problem . . . . . . . . . . . . . . . . 47

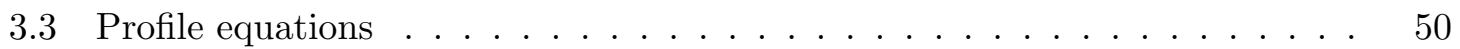

3.4 Error analysis . . . . . . . . . . . . . . . . . . 58

\section{Introduction}

In this paper we study highly oscillatory solutions of quasilinear hyperbolic fixed and free boundary problems for a general class of equations that includes the compressible Euler equations. Several natural (fixed) boundary conditions for the compressible Euler equations are discussed in Example 1.7. Two free boundary problems are those associated with the construction of multidimensional shocks $[13,12]$ and vortex sheets [1]; here we treat the case of shocks. Our main objective is to construct leading order weakly nonlinear geometric optics expansions of the solutions (which are valuable because, for example, they exhibit important qualitative properties), and to rigorously justify such expansions, that is, to show that they are close in a precise sense to true exact solutions. The analogous problem for oscillatory solutions of initial-value problems in free space was solved by Joly, Métivier, and Rauch in [8]. The present paper, together with the companion paper [23], arose from an attempt to understand the obstacles in applying the methods of [8] to boundary problems.

Weakly nonlinear geometric optics expansions for oscillating multidimensional shocks were constructed formally in [14] and then rigorously justified in [21]. For shocks in one space dimension a justification was provided in [4, 19]. The method used in [21], which applies also to the easier case of fixed boundaries, required the construction of high-order expansions involving profiles $\mathcal{U}^{j}$ of amplitude $\epsilon^{j}, j=0, \ldots, M$, for $M$ large (along with a similar expansion for the function defining the shock front). The construction of the profiles $\mathcal{U}^{j}, j \geq 1$, depended on the assumption that the perturbing boundary frequency $(\beta$ in (1.2)) was chosen to avoid the occurrence of small divisors (see Remark 2.26). However, the construction of the leading profile $\mathcal{U}^{0}$ does not require such an assumption. The small divisor assumption, although valid for "generic" $\beta$, is difficult to check for any given $\beta$. Since the leading profile already contains all the important qualitative information that one hopes to obtain from geometric optics, it is preferable to have a rigorous justification of leading term expansions that requires no small divisor assumption. ${ }^{1}$

\footnotetext{
${ }^{1}$ Another advantage to working with leading term expansions is that it is no longer necessary as in [21] to work with carefully chosen "adapted bases" for the space of phases.
} 
The method we use here to justify leading term expansions involves replacing the original system (1.2) with an associated singular system (1.5) involving coefficients of order $\frac{1}{\epsilon}$ and a new unknown $U_{\epsilon}\left(x, \theta_{0}\right)$ periodic in $\theta_{0}$. Exact solutions $U_{\epsilon}$ to the singular system yield exact solutions to the original system by a substitution

$$
u_{\epsilon}(x)=U_{\epsilon}\left(x, \frac{\phi_{0}\left(x^{\prime}\right)}{\epsilon}\right) .
$$

Both the singular system and the system of profile equations satisfied by the leading profile $\mathcal{U}^{0}\left(x, \theta_{0}, \xi_{d}\right)$, which is almost periodic in $\left(\theta_{0}, \xi_{d}\right)$, are solved by Picard iteration. The error analysis is based on "simultaneous Picard iteration", where the idea is to show that for every $n$, the $n$-th profile iterate $\mathcal{U}^{0, n}\left(x, \theta_{0}, \frac{x_{d}}{\epsilon}\right)$ converges as $\epsilon \rightarrow 0$ in an appropriate sense to the $n$-th exact iterate $U_{\epsilon}^{n}\left(x, \theta_{0}\right)$, and to conclude therefrom that $\mathcal{U}^{0}\left(x, \theta_{0}, \frac{x_{d}}{\epsilon}\right)$ is close to $U_{\epsilon}\left(x, \theta_{0}\right)$ for $\epsilon$ small. Below we describe what is involved in adapting this method, which was first used by [8] for initial-value problems, to problems with boundaries.

\subsection{Exact solutions and singular systems.}

In order to study geometric optics for nonlinear problems with highly oscillatory solutions it is important first to settle the question of whether exact solutions exist on a fixed time interval independent of the wavelength ( $\epsilon$ in the notation below). A powerful method for studying this problem, introduced in [8] for initial value problems and extended to boundary problems in [23], is to replace the original system with an associated singular system.

On $\overline{\mathbb{R}}_{+}^{d+1}=\left\{x=\left(x^{\prime}, x_{d}\right)=\left(t, y, x_{d}\right)=\left(t, x^{\prime \prime}\right): x_{d} \geq 0\right\}$ consider the $N \times N$ quasilinear hyperbolic boundary problem:

$$
\begin{aligned}
& \sum_{j=0}^{d} A_{j}\left(v_{\epsilon}\right) \partial_{x_{j}} v_{\epsilon}=f\left(v_{\epsilon}\right) \\
& \left.b\left(v_{\epsilon}\right)\right|_{x_{d}=0}=g_{0}+\epsilon G\left(x^{\prime}, \frac{x^{\prime} \cdot \beta}{\epsilon}\right) \\
& v_{\epsilon}=u_{0} \text { in } t<0,
\end{aligned}
$$

where $x_{0}=t$ is time, $G\left(x^{\prime}, \theta_{0}\right) \in C^{\infty}\left(\mathbb{R}^{d} \times \mathbb{T}^{1}, \mathbb{R}^{p}\right)$ is periodic in $\theta_{0}$ with supp $G \subset\left\{x_{0} \geq 0\right\}$, and the boundary frequency $\beta \in \mathbb{R}^{d} \backslash 0$. Here the coefficients $A_{j} \in C^{\infty}\left(\mathbb{R}^{N}, \mathbb{R}^{N^{2}}\right), f \in$ $C^{\infty}\left(\mathbb{R}^{N}, \mathbb{R}^{N}\right)$, and $b \in C^{\infty}\left(\mathbb{R}^{N}, \mathbb{R}^{p}\right)$.

Looking for $v_{\epsilon}$ as a perturbation $v_{\epsilon}=u_{0}+\epsilon u_{\epsilon}$ of a constant state $u_{0}$ such that $f\left(u_{0}\right)=0$, $b\left(u_{0}\right)=g_{0}$, we obtain for $u_{\epsilon}$ the system (with slightly different $A_{j}$ ) 


$$
\begin{aligned}
& \text { (a) } P\left(\epsilon u_{\epsilon}, \partial_{x}\right) u_{\epsilon}:=\sum_{j=0}^{d} A_{j}\left(\epsilon u_{\epsilon}\right) \partial_{x_{j}} u_{\epsilon}=\mathcal{F}\left(\epsilon u_{\epsilon}\right) u_{\epsilon} \text { on } x_{d} \geq 0 \\
& \text { (b) }\left.B\left(\epsilon u_{\epsilon}\right) u_{\epsilon}\right|_{x_{d}=0}=G\left(x^{\prime}, \frac{x^{\prime} \cdot \beta}{\epsilon}\right) \\
& \text { (c) } u_{\epsilon}=0 \text { in } t<0,
\end{aligned}
$$

where $B(v)$ is a $C^{\infty} p \times N$ real matrix defined by

$$
b\left(u_{0}+\epsilon u_{\epsilon}\right)=b\left(u_{0}\right)+B\left(\epsilon u_{\epsilon}\right) \epsilon u_{\epsilon}
$$

and $\mathcal{F}$ is defined similarly. We assume that the boundary $\left\{x_{d}=0\right\}$ is noncharacteristic, that is, $A_{d}(0)$ is invertible. The other key assumptions, explained in section 1.2, are that $P\left(0, \partial_{x}\right)$ is hyperbolic with characteristics of constant multiplicity (Assumption 1.1) and that $\left(P\left(0, \partial_{x}\right), B(0)\right)$ is uniformly stable (Assumption 1.6).

For any fixed $\epsilon_{0}>0$ the standard theory of hyperbolic boundary problems (see e.g., $[3,9])$ yields solutions of $(1.3)$ on a fixed time interval $\left[0, T_{\epsilon_{0}}\right]$ independent of $\epsilon \geq \epsilon_{0}$. However, since Sobolev norms of the boundary data blow up as $\epsilon \rightarrow 0$, the standard theory yields solutions $u_{\epsilon}$ of (1.3) only on time intervals $\left[0, T_{\epsilon}\right]$ that shrink to zero as $\epsilon \rightarrow 0$. In [23] exact (and necessarily unique) solutions to (1.3) of the form $u_{\epsilon}(x)=U_{\epsilon}\left(x, \frac{x^{\prime} \cdot \beta}{\epsilon}\right)$ were constructed on a time interval independent of $\epsilon \in\left(0, \epsilon_{0}\right]$ for $\epsilon_{0}$ sufficiently small, where $U_{\epsilon}\left(x, \theta_{0}\right)$ is periodic in $\theta_{0}$ and satisfies the singular system derived by substituting $U_{\epsilon}\left(x, \frac{x^{\prime} \cdot \beta}{\epsilon}\right)$ into (1.3):

$$
\begin{aligned}
& \sum_{j=0}^{d} A_{j}\left(\epsilon U_{\epsilon}\right) \partial_{x_{j}} U_{\epsilon}+\frac{1}{\epsilon} \sum_{j=0}^{d-1} A_{j}\left(\epsilon U_{\epsilon}\right) \beta_{j} \partial_{\theta_{0}} U_{\epsilon}=\mathcal{F}\left(\epsilon U_{\epsilon}\right) U_{\epsilon}, \\
& \left.B\left(\epsilon U_{\epsilon}\right)\left(U_{\epsilon}\right)\right|_{x_{d}=0}=G\left(x^{\prime}, \theta_{0}\right), \\
& U_{\epsilon}=0 \text { in } t<0 .
\end{aligned}
$$

Let us compare (1.5) to the singular system studied in [8]. Consider the initial value problem obtained by posing (1.3) on $\mathbb{R}^{d+1}$ and replacing (1.3)(b) and (c) by the initial condition $u_{\epsilon}\left(0, x^{\prime \prime}\right)=H\left(x^{\prime \prime}, \frac{\alpha \cdot x^{\prime \prime}}{\epsilon}\right)$, where $H\left(x^{\prime \prime}, \omega\right)$ is periodic in $\omega$. Looking for a solution of the form $u_{\epsilon}\left(t, x^{\prime \prime}\right)=V_{\epsilon}\left(t, x^{\prime \prime}, \frac{\alpha \cdot x^{\prime \prime}}{\epsilon}\right)$, where $V_{\epsilon}\left(t, x^{\prime \prime}, \omega\right)$ is periodic in $\omega$, one obtains

$$
\begin{aligned}
& \text { (a) } \sum_{j=0}^{d} A_{j}\left(\epsilon V_{\epsilon}\right) \partial_{x_{j}} V_{\epsilon}+\frac{1}{\epsilon} \sum_{j=1}^{d} A_{j}\left(\epsilon V_{\epsilon}\right) \alpha_{j} \partial_{\omega} V_{\epsilon}=\mathcal{F}\left(\epsilon V_{\epsilon}\right) V_{\epsilon}, \\
& \text { (b) }\left.V_{\epsilon}\right|_{t=0}=H\left(x^{\prime \prime}, \omega\right) .
\end{aligned}
$$

Under the assumption that the matrices $A_{j}$ were symmetric (and $A_{0}=I$ ), [8] proved existence of $V_{\epsilon}$ on a fixed time interval independent of $\epsilon$ by an argument based on an $L^{2}$ 
estimate (really an estimate of $\left|V_{\epsilon}\right|_{C\left(t, L^{2}\left(x^{\prime \prime}, \omega\right)\right)}$ ) derived in the usual way by pairing (1.6)(a) with $V_{\epsilon}$ and integrating by parts. The key observation is that the singular terms of size $O\left(\frac{1}{\epsilon}\right)$ cancel out due to the symmetry hypothesis. Higher derivative estimates of norms $\left|V_{\epsilon}\right|_{C\left(t, H^{s}\left(x^{\prime \prime}, \omega\right)\right)}$ are then readily obtained (in the coherent case of linear phases considered here) by commuting $\partial_{x^{\prime \prime}, \omega}^{\alpha}$ derivatives through the equation and initial condition. For $s>$ $\frac{d+1}{2}$ the space $C\left(t, H^{s}\left(x^{\prime \prime}, \omega\right)\right)$ contains $L^{\infty}$ and is a Banach algebra, and so by a standard Picard iteration one obtains solutions of (1.6) in $C\left(t, H^{s+1}\left(x^{\prime \prime}, \omega\right)\right)$ on a fixed time interval independent of $\epsilon \in\left(0, \epsilon_{0}\right]$.

Attempts to apply this approach to the singular boundary problem (1.5) encounter serious obstacles. Even if one assumes that the matrices $A_{j}$ are symmetric (as we do not here), there is no way to obtain an $L^{2}$ estimate uniform in $\epsilon$ by a simple integration by parts because of the boundary terms that arise. ${ }^{2}$ Moreover, while the spaces $C\left(t, H^{s}\left(x^{\prime \prime}, \omega\right)\right)$ work well for initial-value problems and suggest that one should try the analogous spaces $C\left(x_{d}, H^{s}\left(x^{\prime}, \theta_{0}\right)\right)$ for the boundary problem (1.5), the blow-up examples of [22] show that for certain boundary frequencies $\beta$ it is impossible to estimate solutions of (1.5) uniformly with respect to $\epsilon$ in $C\left(x_{d}, H^{s}\left(x^{\prime}, \theta_{0}\right)\right)$ norms, or indeed in any norm that dominates the $L^{\infty}$ norm. ${ }^{3}$.

In [23] a class of singular pseudodifferential operators, acting on functions $U\left(x^{\prime}, \theta_{0}\right)$ periodic in $\theta_{0}$ and having the form

$$
p_{s}\left(D_{x^{\prime}, \theta_{0}}\right) U=\sum_{m \in \mathbb{Z}} \int e^{i x^{\prime} \xi^{\prime}+i \theta_{0} m} p\left(\epsilon V\left(x^{\prime}, \theta_{0}\right), \xi^{\prime}+\frac{m \beta}{\epsilon}, \gamma\right) \widehat{U}\left(\xi^{\prime}, m\right) d \xi^{\prime}, \quad \gamma \geq 1
$$

was introduced to deal with these difficulties. Observe that after multiplication by $A_{d}^{-1}\left(\epsilon U_{\epsilon}\right)$ and setting $\tilde{A}_{j}:=A_{d}^{-1} A_{j}, F=A_{d}^{-1} \mathcal{F},(1.5)$ becomes

$$
\begin{aligned}
& \partial_{x_{d}} U_{\epsilon}+\sum_{j=0}^{d-1} \tilde{A}_{j}\left(\epsilon U_{\epsilon}\right)\left(\partial_{x_{j}}+\frac{\beta_{j} \partial_{\theta_{0}}}{\epsilon}\right) U_{\epsilon} \\
& \quad \equiv \partial_{x_{d}} U_{\epsilon}+\mathbb{A}\left(\epsilon U_{\epsilon}, \partial_{x^{\prime}}+\frac{\beta \partial_{\theta_{0}}}{\epsilon}\right) U_{\epsilon}=F\left(\epsilon U_{\epsilon}\right) U_{\epsilon}, \\
& \left.B\left(\epsilon U_{\epsilon}\right)\left(U_{\epsilon}\right)\right|_{x_{d}=0}=G\left(x^{\prime}, \theta_{0}\right), \\
& U_{\epsilon}=0 \text { in } t<0,
\end{aligned}
$$

where $\mathbb{A}\left(\epsilon U_{\epsilon}, \partial_{x^{\prime}}+\frac{\beta \partial_{\theta_{0}}}{\epsilon}\right)$ is a (differential) operator that can be expressed in the form (1.7). Kreiss-type symmetrizers $r_{s}\left(D_{x^{\prime}, \theta_{0}}\right)$ in the singular calculus can be constructed for the system (1.8) as in [23] under the assumptions given below. With these one can prove

\footnotetext{
${ }^{2}$ The class of symmetric problems with maximal strictly dissipative boundary conditions provides an exception to this statement, but that class is too restrictive for our purposes; for example, the free boundary problem for multi-D shocks does not lie in this class.

${ }^{3}$ The problem occurs only for $\beta$ in the glancing set (Definition 1.3), as the examples of [22] together with the results of [23] show.
} 
$L^{2}\left(x_{d}, H^{s}\left(x^{\prime}, \theta_{0}\right)\right)$ estimates uniform in $\epsilon$ for the linearization of (1.8). To progress further and control $L^{\infty}$ norms, the boundary frequency $\beta$ must be restricted to the complement of the glancing set (Definition 1.3). With this extra assumption the singular calculus was used in [23] to block-diagonalize the operator $\mathbb{A}\left(\epsilon U_{\epsilon}, \partial_{x^{\prime}}+\frac{\beta \partial_{\theta_{0}}}{\epsilon}\right)$ and thereby prove estimates uniform with respect to $\epsilon$ in the spaces

$$
E_{T}^{s}=C\left(x_{d}, H_{T}^{s}\left(x^{\prime}, \theta_{0}\right)\right) \cap L^{2}\left(x_{d}, H_{T}^{s+1}\left(x^{\prime}, \theta_{0}\right)\right) .
$$

These spaces are algebras and contain $L^{\infty}$ for $s>\frac{d+1}{2}$. For large enough $s$, as determined by the requirements of the calculus, existence of solutions to (1.8) in $E_{T}^{s}$ on a time interval $[0, T]$ independent of $\epsilon \in\left(0, \epsilon_{0}\right]$ follows by Picard iteration (see Theorem 1.13).

Although the singular calculus will not be used in this paper, the spaces $E_{T}^{s}$ and the associated estimates, which are recalled in Proposition 2.23 for fixed boundaries and in Proposition 3.15 for shocks, play an essential role.

Other approaches to justifying nonlinear geometric optics expansions on domains with boundary are used by Lescarret [11], who considers dispersive media, maximally dissipative boundary conditions, and possibly characteristic boundaries, and Marcou [15], who treats a maximally dissipative boundary condition for which the uniform Lopatinski condition fails in the elliptic region.

\subsection{Assumptions and main results.}

Before continuing with an overview of the strategies for constructing profiles and for showing that approximate solutions are close to exact solutions, we pause to give a precise statement of our assumptions and main results.

We make the following hyperbolicity assumption on the system (1.3):

Assumption 1.1. The matrix $A_{0}=I$. For an open neighborhood $\mathcal{O}$ of $0 \in \mathbb{R}^{N}$, there exists an integer $q \geq 1$, some real functions $\lambda_{1}, \ldots, \lambda_{q}$ that are $C^{\infty}$ on $\mathcal{O} \times \mathbb{R}^{d} \backslash\{0\}$ and homogeneous of degree 1 and analytic in $\xi$, and there exist some positive integers $\nu_{1}, \ldots, \nu_{q}$ such that:

$$
\operatorname{det}\left[\tau I+\sum_{j=1}^{d} \xi_{j} A_{j}(u)\right]=\prod_{k=1}^{q}\left(\tau+\lambda_{k}(u, \xi)\right)^{\nu_{k}}
$$

for $u \in \mathcal{O}, \xi=\left(\xi_{1}, \ldots, \xi_{d}\right) \in \mathbb{R}^{d} \backslash\{0\}$. Moreover the eigenvalues $\lambda_{1}(u, \xi), \ldots, \lambda_{q}(u, \xi)$ are semi-simple (their algebraic multiplicity equals their geometric multiplicity) and satisfy $\lambda_{1}(u, \xi)<\cdots<\lambda_{q}(u, \xi)$ for all $u \in \mathcal{O}, \xi \in \mathbb{R}^{d} \backslash\{0\}$.

We restrict our analysis to noncharacteristic boundaries and therefore make the following:

Assumption 1.2. For $u \in \mathcal{O}$ the matrix $A_{d}(u)$ is invertible and the matrix $B(u)$ has maximal rank, its rank $p$ being equal to the number of positive eigenvalues of $A_{d}(u)$ (counted with their multiplicity). 
In the normal modes analysis for the linearization of $(1.3)$ at $0 \in \mathcal{O}$, one first performs a Laplace transform in the time variable $t$ and a Fourier transform in the tangential space variables $y$. We let $\tau-i \gamma \in \mathbb{C}$ and $\eta \in \mathbb{R}^{d-1}$ denote the dual variables of $t$ and $y$. We introduce the symbol

$$
\mathcal{A}(\zeta):=-i A_{d}^{-1}(0)\left((\tau-i \gamma) I+\sum_{j=1}^{d-1} \eta_{j} A_{j}(0)\right), \quad \zeta:=(\tau-i \gamma, \eta) \in \mathbb{C} \times \mathbb{R}^{d-1} .
$$

For future use, we also define the following sets of frequencies:

$$
\begin{array}{lr}
\Xi:=\left\{(\tau-i \gamma, \eta) \in \mathbb{C} \times \mathbb{R}^{d-1} \backslash(0,0): \gamma \geq 0\right\}, \quad \Sigma:=\left\{\zeta \in \Xi: \tau^{2}+\gamma^{2}+|\eta|^{2}=1\right\}, \\
\Xi_{0}:=\left\{(\tau, \eta) \in \mathbb{R} \times \mathbb{R}^{d-1} \backslash(0,0)\right\}=\Xi \cap\{\gamma=0\}, & \Sigma_{0}:=\Sigma \cap \Xi_{0} .
\end{array}
$$

Henceforth we suppress the $u$ in $\lambda_{k}(u, \xi)$ when it is evaluated at $u=0$ and write $\lambda_{k}(0, \xi)=$ $\lambda_{k}(\xi)$. Two key objects in our analysis are the hyperbolic region and the glancing set that are defined as follows:

Definition 1.3. - The hyperbolic region $\mathcal{H}$ is the set of all $(\tau, \eta) \in \Xi_{0}$ such that the matrix $\mathcal{A}(\tau, \eta)$ is diagonalizable with purely imaginary eigenvalues.

- Let $G$ denote the set of all $(\tau, \xi) \in \mathbb{R} \times \mathbb{R}^{d}$ such that $\xi \neq 0$ and there exists an integer $k \in\{1, \ldots, q\}$ satisfying:

$$
\tau+\lambda_{k}(\xi)=\frac{\partial \lambda_{k}}{\partial \xi_{d}}(\xi)=0 .
$$

If $\pi(G)$ denotes the projection of $G$ on the d first coordinates (in other words $\pi(\tau, \xi)=$ $\left(\tau, \xi_{1}, \ldots, \xi_{d-1}\right)$ for all $\left.(\tau, \xi)\right)$, the glancing set $\mathcal{G}$ is $\mathcal{G}:=\pi(G) \subset \Xi_{0}$.

We recall the following result that is due to Kreiss [9] in the strictly hyperbolic case (when all integers $\nu_{j}$ in Assumption 1.1 equal 1) and to Métivier [17] in our more general framework:

Proposition $1.4([9,17])$. Let Assumptions 1.1 and 1.2 be satisfied. Then for all $\zeta \in$ $\Xi \backslash \Xi_{0}$, the matrix $\mathcal{A}(\zeta)$ has no purely imaginary eigenvalue and its stable subspace $\mathbb{E}^{s}(\zeta)$ has dimension $p$. Furthermore, $\mathbb{E}^{s}$ defines an analytic vector bundle over $\Xi \backslash \Xi_{0}$ that can be extended as a continuous vector bundle over $\Xi$.

For all $(\tau, \eta) \in \Xi_{0}$, we let $\mathbb{E}^{s}(\tau, \eta)$ denote the continuous extension of $\mathbb{E}^{s}$ to the point $(\tau, \eta)$. The analysis in [17] shows that away from the glancing set $\mathcal{G} \subset \Xi_{0}, \mathbb{E}^{s}(\zeta)$ depends analytically on $\zeta$, and the hyperbolic region $\mathcal{H}$ does not contain any glancing point.

Next we define the hyperbolic operator

$$
L\left(\partial_{x}\right):=\partial_{t}+\sum_{j=1}^{d} A_{j}(0) \partial_{x_{j}}
$$

and recall the definition of uniform stability $[9,3]$ : 
Definition 1.5. The problem (1.3) is uniformly stable at $u=0$ if the linearized operators $\left(L\left(\partial_{x}\right), B(0)\right)$ at $u=0$ are such that

$$
B(0): \mathbb{E}^{s}(\tau, \eta) \rightarrow \mathbb{C}^{p} \text { is an isomorphism for all }(\tau, \eta) \in \Sigma .
$$

Assumption 1.6. The problem (1.3) is uniformly stable at $u=0$.

It is clear that uniform stability at $u=0$ implies uniform stability at nearby states. Thus, there is a slight redundancy in Assumptions 1.2 and 1.6.

Example 1.7 (Euler equations). Consider the isentropic, compressible Euler equations in three space dimensions on the half space $\left\{x_{3} \geq 0\right\}$, where the unknowns are density $\rho$ and velocity $u=\left(u_{1}, u_{2}, u_{3}\right)$ :

$$
\partial_{t}\left(\begin{array}{c}
\rho \\
\rho u_{1} \\
\rho u_{2} \\
\rho u_{3}
\end{array}\right)+\partial_{x_{1}}\left(\begin{array}{c}
\rho u_{1} \\
\rho u_{1}^{2}+p(\rho) \\
\rho u_{2} u_{1} \\
\rho u_{3} u_{1}
\end{array}\right)+\partial_{x_{2}}\left(\begin{array}{c}
\rho u_{2} \\
\rho u_{1} u_{2} \\
\rho u_{2}^{2}+p(\rho) \\
\rho u_{3} u_{2}
\end{array}\right)+\partial_{x_{3}}\left(\begin{array}{c}
\rho u_{3} \\
\rho u_{1} u_{3} \\
\rho u_{2} u_{3} \\
\rho u_{3}^{2}+p(\rho)
\end{array}\right)=\left(\begin{array}{l}
0 \\
0 \\
0 \\
0
\end{array}\right) .
$$

The hyperbolicity assumption is satisfied in the region of state space where $\rho>0, c^{2}=$ $p^{\prime}(\rho)>0$, where $p(\rho)$ is the pressure. The eigenvalues $\lambda_{k}(\rho, u, \xi)$ are then

$$
\lambda_{1}=u \cdot \xi-c|\xi|, \lambda_{2}=u \cdot \xi, \lambda_{3}=u \cdot \xi+c|\xi|, \text { with }\left(\nu_{1}, \nu_{2}, \nu_{3}\right)=(1,2,1) .
$$

The boundary is noncharacteristic for the system obtained by linearizing around any constant state $(\rho, u)$ (which now corresponds to the state denoted $u_{0}$ in (1.2)) with $u_{3} \notin$ $\{0, c,-c\}$.

To discuss Assumption 1.6 we consider the following cases. In each case the boundary condition chosen is the natural "residual boundary condition" that arises in the small viscosity limit of the compressible Navier-Stokes equations with Dirichlet boundary conditions. We refer to [6], section 5 for complete proofs and a full discussion of the statements below.

(a)Subsonic outflow: $u_{3}<0,\left|u_{3}\right|<c$. In this case the number of positive eigenvalues of $A_{3}(\rho, u)$ is $p=1$, so we need one scalar boundary condition. Taking $\phi$ in (1.2) to be $\phi(\rho, u)=u_{3}$, we have $B(0)=\left[\begin{array}{llll}0 & 0 & 0 & 1\end{array}\right]$, and this boundary condition satisfies Assumption 1.6.

(b)Subsonic inflow: $0<u_{3}<c$. Now $p=3$ and the residual boundary condition is $\phi(\rho, u)=\left(\rho u_{3}, u_{1}, u_{2}\right)$. The linearized operator

$$
B(0)(\dot{\rho}, \dot{u})=\left(\rho \dot{u}_{3}+\dot{\rho} u_{3}, \dot{u}_{1}, \dot{u}_{2}\right)
$$

satisfies Assumption 1.6.

(c) Supersonic inflow: $0<c<u_{3}$. This is a trivial case where $p=4$ and $B(0)$ is the $4 \times 4$ identity matrix, so Assumption 1.6 holds.

(d)Supersonic outflow: $u_{3}<0,\left|u_{3}\right|>c$. This is another trivial case where $p=0$, so $B(0)$ is absent and Assumption 1.6 holds vacuously. 
If we take $u=\left(u_{1}, u_{2}, u_{3}\right)$ with $\left|u_{3}\right|<c$, the hyperbolic region is given by

$$
\mathcal{H}=\left\{(\tau, \eta) \in \mathbb{R}^{3}:\left|\tau+u_{1} \eta_{1}+u_{2} \eta_{2}\right|>\sqrt{c^{2}-u_{3}^{2}}|\eta|\right\} .
$$

If $\left|u_{3}\right|>c$ then $\mathcal{H}=\mathbb{R}^{3} \backslash 0$.

With obvious modifications the same statements hold for $2 D$ Euler which, unlike $3 D$ Euler, is strictly hyperbolic. ${ }^{4}$

Boundary and interior phases. We consider a planar real phase $\phi_{0}$ defined on the boundary:

$$
\phi_{0}(t, y):=\underline{\tau} t+\underline{\eta} \cdot y, \quad(\underline{\tau}, \underline{\eta}) \in \Xi_{0} .
$$

As follows from earlier works (e.g. [14]), oscillations on the boundary associated with the phase $\phi_{0}$ give rise to oscillations in the interior associated with some planar phases $\phi_{m}$. These phases are characteristic for the hyperbolic operator $L\left(\partial_{x}\right)$ and their trace on the boundary equals $\phi_{0}$. For now we make the following:

Assumption 1.8. The phase $\phi_{0}$ defined by (1.18) satisfies $(\underline{\tau}, \eta) \in \mathcal{H}$.

Thanks to Assumption 1.8, we know that the matrix $\mathcal{A}(\underline{\tau}, \underline{\eta})$ is diagonalizable with purely imaginary eigenvalues. These eigenvalues are denoted $i \underline{\omega}_{1}, \ldots, i \underline{\omega}_{M}$, where the $\underline{\omega}_{m}$ 's are real and pairwise distinct. The $\underline{\omega}_{m}$ 's are the roots (and all the roots are real) of the dispersion relation:

$$
\operatorname{det}\left[\underline{\tau} I+\sum_{j=1}^{d-1} \underline{\eta}_{j} A_{j}(0)+\omega A_{d}(0)\right]=0 .
$$

To each root $\underline{\omega}_{m}$ there corresponds a unique integer $k_{m} \in\{1, \ldots, q\}$ such that $\underline{\tau}+\lambda_{k_{m}}\left(\underline{\eta}, \underline{\omega}_{m}\right)=$ 0 . We can then define the following real ${ }^{5}$ phases and their associated group velocities:

$$
\forall m=1, \ldots, M, \quad \phi_{m}(x):=\phi_{0}(t, y)+\underline{\omega}_{m} x_{d}, \quad \mathbf{v}_{m}:=\nabla \lambda_{k_{m}}\left(\underline{\eta}, \underline{\omega}_{m}\right) .
$$

Let us observe that each group velocity $\mathbf{v}_{m}$ is either incoming or outgoing with respect to the space domain $\mathbb{R}_{+}^{d}$ : the last coordinate of $\mathbf{v}_{m}$ is nonzero. This property holds because $(\underline{\tau}, \eta)$ does not belong to the glancing set $\mathcal{G}$. We can therefore adopt the following classification:

Definition 1.9. The phase $\phi_{m}$ is incoming if the group velocity $\mathbf{v}_{m}$ is incoming (that is, $\left.\partial_{\xi_{d}} \lambda_{k_{m}}\left(\underline{\eta}, \underline{\omega}_{m}\right)>0\right)$, and it is outgoing if the group velocity $\mathbf{v}_{m}$ is outgoing $\left(\partial_{\xi_{d}} \lambda_{k_{m}}\left(\underline{\eta}, \underline{\omega}_{m}\right)<\right.$ $0)$.

\footnotetext{
${ }^{4}$ The boundary conditions in Example 1.7 are, in fact, maximally strictly dissipative. The RankineHugoniot condition for shocks (more precisely, the reduced boundary condition after the front is eliminated as in [13]) provides an example of a uniformly stable condition (Definition 3.1) that is not maximally dissipative. Other examples are given in Chapter 14 of Benzoni-Serre [2].

${ }^{5}$ If $(\underline{\tau}, \underline{\eta})$ does not belong to the hyperbolic region $\mathcal{H}$, some of the phases $\varphi_{m}$ may be complex, see e.g. $[20,22,1 \overline{1}, 15]$. Moreover, glancing phases introduce a new scale $\sqrt{\epsilon}$ as well as boundary layers.
} 
In all that follows, we let $\mathcal{I}$ denote the set of indices $m \in\{1, \ldots, M\}$ such that $\phi_{m}$ is an incoming phase, and $\mathcal{O}$ denote the set of indices $m \in\{1, \ldots, M\}$ such that $\phi_{m}$ is an outgoing phase. If $p \geq 1$, then $\mathcal{I}$ is nonempty, while if $p \leq N-1, \mathcal{O}$ is nonempty. The following well-known lemma, whose proof is recalled in [5], gives a useful decomposition of $\mathbb{E}^{s}$ in the hyperbolic region.

Lemma 1.10. The stable subspace $\mathbb{E}^{s}(\underline{\tau}, \underline{\eta})$ admits the decomposition:

$$
\mathbb{E}^{s}(\underline{\tau}, \underline{\eta})=\oplus_{m \in \mathcal{I}} \operatorname{Ker} L\left(d \phi_{m}\right),
$$

and each vector space in the decomposition (1.20) admits a basis of real vectors.

Main results. We will use the notation:

$$
\begin{aligned}
& L(\tau, \xi):=\tau I+\sum_{j=1}^{d} \xi_{j} A_{j}(0), \\
& \beta=(\underline{\tau}, \underline{\eta}), x^{\prime}=(t, y), \phi_{0}\left(x^{\prime}\right)=\beta \cdot x^{\prime} .
\end{aligned}
$$

For each phase $\phi_{m}, d \phi_{m}$ denotes the differential of the function $\phi_{m}$ with respect to its argument $x=\left(t, y, x_{d}\right)$. It follows from Assumption 1.1 that the eigenspace of $\mathcal{A}(\beta)$ associated with the eigenvalue $i \underline{\omega}_{m}$ coincides with the kernel of $L\left(d \phi_{m}\right)$ and has dimension $\nu_{k_{m}}$. The next Lemma, proved in [5], gives a useful decomposition of $\mathbb{C}^{N}$ and introduces projectors needed later for formulating and solving the profile equations.

Lemma 1.11. The space $\mathbb{C}^{N}$ admits the decomposition:

$$
\mathbb{C}^{N}=\oplus_{m=1}^{M} \operatorname{Ker} L\left(d \phi_{m}\right)
$$

and each vector space in (1.22) admits a basis of real vectors. If we let $P_{1}, \ldots, P_{M}$ denote the projectors associated with the decomposition (1.22), then for all $m=1, \ldots, M$, there holds $\operatorname{Im} A_{d}^{-1} L\left(d \phi_{m}\right)=\operatorname{Ker} P_{m}$.

For each $m \in\{1, \ldots, M\}$ we let

$$
r_{m, k}, k=1, \ldots, \nu_{k_{m}}
$$

denote a basis of ker $L\left(d \phi_{m}\right)$ consisting of real vectors. In section 2.1 we construct an approximate solution $u_{\epsilon}^{a}$ of (1.3) of the form

$$
u_{\epsilon}^{a}(x)=\underline{v}(x)+\sum_{m=1}^{M} \sum_{k=1}^{\nu_{k_{m}}} \sigma_{m, k}\left(x, \frac{\phi_{m}}{\epsilon}\right) r_{m, k}:=\underline{v}(x)+\sum_{m=1}^{M} \mathcal{V}_{m}^{0}\left(x, \frac{\phi_{m}}{\epsilon}\right),
$$

where the $\sigma_{m, k}\left(x, \theta_{m}\right)$ are $C^{1}$ functions periodic in $\theta_{m}$ with mean 0 which describe the propagation of oscillations with group velocity $\mathbf{v}_{m}$ (see Propositions 2.19 and 2.21). Observe that if one plugs $u_{\epsilon}^{a}$ into $P\left(\epsilon u_{\epsilon}, \partial_{x}\right) u_{\epsilon}$, the terms of order $\frac{1}{\epsilon}$ vanish, leaving an $O(1)$ error, 
regardless of how $\underline{v}$ and the $\sigma_{m, k}$ are chosen. The interior profile equations satisfied by these functions are solvability conditions that permit this $O(1)$ error to be (at least partially) removed. Additional conditions on the profiles come, of course, from the boundary conditions.

For use in the remainder of the introduction and later, we collect some notation here.

Notations 1.12. (a) Let $\Omega=\overline{\mathbb{R}}_{+}^{d+1} \times \mathbb{T}^{1}, \Omega_{T}=\Omega \cap\{-\infty<t<T\}$, $b \Omega=\mathbb{R}^{d} \times \mathbb{T}^{1}$, $b \Omega_{T}=b \Omega \cap\{-\infty<t<T\}$, and set $\omega_{T}=\overline{\mathbb{R}}_{+}^{d+1} \cap\{-\infty<t<T\}$.

(b) For $s \geq 0$ let $H^{s} \equiv H^{s}(b \Omega)$, the standard Sobolev space with norm $\left\langle V\left(x^{\prime}, \theta_{0}\right)\right\rangle_{s}$.

(c) $L^{2} H^{s} \equiv L^{2}\left(x_{d}, H^{s}(b \Omega)\right)$ with $\left|U\left(x, \theta_{0}\right)\right|_{L^{2} H^{s}} \equiv|U|_{0, s}$.

(d) $C H^{s} \equiv C\left(x_{d}, H^{s}(b \Omega)\right)$ with $\left|U\left(x, \theta_{0}\right)\right|_{C H^{s}} \equiv \sup _{x_{d} \geq 0}\left|U\left(., x_{d}, .\right)\right|_{H^{s}} \equiv|U|_{\infty, s}$ (note that $\left.C H^{s} \subset L^{\infty} H^{s}\right)$.

(e) Similarly, $H_{T}^{s} \equiv H^{s}\left(b \Omega_{T}\right)$ with norm $\langle V\rangle_{s, T}$ and $L^{2} H_{T}^{s} \equiv L^{2}\left(x_{d}, H_{T}^{s}\right), C H_{T}^{s} \equiv$ $C\left(x_{d}, H_{T}^{s}\right)$ have norms $|U|_{0, s, T},|U|_{\infty, s, T}$ respectively.

(f) When the domains of $x_{d}$ and $\left(x^{\prime}, \theta_{0}\right)$ are clear, we sometimes use the self-explanatory notation $C\left(x_{d}, H^{s}\left(x^{\prime}, \theta_{0}\right)\right)$ or $L^{2}\left(x_{d}, H^{s}\left(x^{\prime}, \theta_{0}\right)\right)$.

(g) For $r \geq 0[r]$ is the smallest integer $\geq r$.

(h) $M_{0}=2(d+2)+1$

(i) Given a periodic function $f(x, \theta)$ of $\theta \in \mathbb{R}^{k}$, we write $f(x, \theta)=\underline{f}(x)+f^{*}(x, \theta)$, where $f^{*}$ has mean zero.

Next, we recall the main result of [23], already described in section 1.1, which gives the existence of exact solutions to (1.3) on a time interval independent of $\epsilon$ :

Theorem 1.13 ([23]). (a) Under Assumptions 1.1, 1.2, 1.6, 1.8 consider the quasilinear boundary problem (1.3), where $G\left(x^{\prime}, \theta_{0}\right) \in H^{s+1}(b \Omega), s \geq\left[M_{0}+\frac{d+1}{2}\right]$ has compact support in $x^{\prime}$ and satisfies

$$
\operatorname{supp} G \subset\{t \geq 0\} \text {. }
$$

There exist an $\epsilon_{0}>0, a T_{0}$ independent of $\epsilon \in\left(0, \epsilon_{0}\right]$, and a unique $U_{\epsilon}\left(x, \theta_{0}\right) \in C H_{T_{0}}^{s} \cap$ $L^{2} H_{T_{0}}^{s+1}$ satisfying the singular problem

$$
\begin{aligned}
& \partial_{x_{d}} U_{\epsilon}+\mathbb{A}\left(\epsilon U_{\epsilon}, \partial_{x^{\prime}}+\frac{\beta \cdot \partial_{\theta_{0}}}{\epsilon}\right) U_{\epsilon}=F\left(\epsilon U_{\epsilon}\right) U_{\epsilon}, \\
& \left.B\left(\epsilon U_{\epsilon}\right)\left(U_{\epsilon}\right)\right|_{x_{d}=0}=G\left(x^{\prime}, \theta_{0}\right), \\
& U_{\epsilon}=0 \text { in } t<0,
\end{aligned}
$$

and such that

$$
u_{\epsilon}(x)=U_{\epsilon}\left(x, \frac{x^{\prime} \cdot \beta}{\epsilon}\right)
$$

is the unique $C^{1}$ solution of (1.3) on $\omega_{T_{0}}$. 
Remark 1.14. The regularity requirement $s \geq\left[M_{0}+\frac{d+1}{2}\right]$ in the above theorem is needed in order to apply the singular pseudodifferential calculus introduced in [23].

We can now state the main result of this paper in the case of fixed boundaries. This theorem is a corollary of the result for singular systems given in Theorem 2.22.

Theorem 1.15. Under the same assumptions as in Theorem 1.13, there exists $T_{0}>0$ and functions $\underline{u} \in C^{1}\left(\omega_{T_{0}}\right), \sigma_{m, k}\left(x, \theta_{m}\right) \in C^{1}\left(\Omega_{T_{0}}\right)$ satisfying profile equations (2.63),(2.64), (2.66) and defining an approximate solution $u_{\epsilon}^{a}$ as in (1.24) such that

$$
\lim _{\epsilon \rightarrow 0} u_{\epsilon}-u_{\epsilon}^{a}=0 \text { in } L^{\infty}\left(\Omega_{T_{0}}\right),
$$

where $u_{\epsilon} \in C^{1}\left(\Omega_{T_{0}}\right)$ is the unique exact solution of (1.3).

Theorem 1.15 can be recast in a form where oscillations originate in initial data at $t=0$ and reflect off the boundary $\left\{x_{d}=0\right\}$. This requires a discussion similar to that given in section 3.2 to justify the reduction of the singular shock problem (3.27) to the forward singular shock problem (3.39), so we omit that discussion here.

In section 3 we prove an analogous theorem for shock waves, Theorem 3.13. In that case there is a separate expansion for the oscillating shock front:

$$
\psi_{\epsilon}\left(x^{\prime}\right) \sim \sigma x_{0}+\epsilon\left(\chi^{0}\left(x^{\prime}\right)+\epsilon \chi^{1}\left(x^{\prime}, \frac{\phi^{0}\left(x^{\prime}\right)}{\epsilon}\right)\right) .
$$

The front $\psi_{\epsilon}$ appears in the shock equations (3.25) only through its differential $d_{x^{\prime}} \psi_{\epsilon}$, so one should seek an expansion of the front where the relevant error approaches zero in the $C^{1}$ norm as $\epsilon \rightarrow 0$ as in (3.84). Moreover, since $\chi^{0}$ has no $\theta_{0}$ dependence, the combination $\chi^{0}\left(x^{\prime}\right)+\epsilon \chi^{1}\left(x^{\prime}, \frac{\phi^{0}\left(x^{\prime}\right)}{\epsilon}\right)$ should be viewed as the "leading term" in the perturbation of the front.

\subsection{From almost periodic profiles to mode-by-mode analysis.}

In the fixed boundary case we shall now derive the form of the profile equations in terms of almost periodic profiles that we must use for the simultaneous Picard iteration argument.

Theorem 1.13 suggests looking for approximate solutions to (1.3) of the form

$$
u_{\epsilon}^{a}(x)=\left.U_{\epsilon}^{a}\left(x, \theta_{0}\right)\right|_{\theta_{0}=\frac{\phi_{0}}{\epsilon}} .
$$

Recalling that in the profiles $\sigma_{m, k}\left(x, \theta_{m}\right)$ of $(1.24), \theta_{m}$ is a placeholder for

$$
\phi_{m}(x) / \epsilon=\left(\phi_{0}+\underline{\omega}_{m} x_{d}\right) / \epsilon,
$$

we see that (1.29) is compatible with the ansatz (1.24) if we introduce $\xi_{d}$ as a placeholder for $\frac{x_{d}}{\epsilon}$ and take

$$
U_{\epsilon}^{a}\left(x, \theta_{0}\right)=\mathcal{U}^{0}\left(x, \theta_{0}, \frac{x_{d}}{\epsilon}\right)
$$


for some profile $\mathcal{U}^{0}\left(x, \theta_{0}, \xi_{d}\right)$ that is periodic in $\theta_{0}$, but just almost-periodic in $\left(\theta_{0}, \xi_{d}\right)$. To derive the profile equations we work formally for now and write $\mathcal{U}^{0}$ as a trigonometric series

$$
\mathcal{U}^{0}\left(x, \theta_{0}, \xi_{d}\right)=\sum_{\kappa=\left(\kappa_{0}, \kappa_{d}\right) \in \mathbb{Z} \times \mathbb{R}} U_{\kappa}(x) e^{i \kappa_{0} \theta_{0}+i \kappa_{d} \xi_{d}} .
$$

Plugging (1.29) into $P\left(\epsilon u_{\epsilon}, \partial_{x}\right) u_{\epsilon}$, we find that the terms of order $\frac{1}{\epsilon}$ vanish if and only if

$$
\mathcal{L}\left(\partial_{\theta_{0}}, \partial_{\xi_{d}}\right) \mathcal{U}^{0}=0, \text { where } \mathcal{L}\left(\partial_{\theta_{0}}, \partial_{\xi_{d}}\right):=L\left(d \phi_{0}\right) \partial_{\theta_{0}}+L\left(d x_{d}\right) \partial_{\xi_{d}} .
$$

This in turn is equivalent to the family of equations

$$
i L\left(\kappa_{0} \beta, \kappa_{d}\right) U_{\kappa}(x)=0 \text { for all } \kappa \in \mathbb{Z} \times \mathbb{R} .
$$

These equations imply that the spectrum of $\mathcal{U}^{0}(x, \cdot, \cdot)$ is contained in the set of characteristic modes

$$
\mathcal{C}=\left\{\kappa \in \mathbb{Z} \times \mathbb{R}: \operatorname{det} L\left(\kappa_{0} \beta, \kappa_{d}\right)=0\right\} .
$$

Recalling the discussion below Assumption 1.8, we have

$$
\mathcal{C}=\cup_{m=1}^{M} \mathcal{C}_{m}, \text { where } \mathcal{C}_{m}=\left\{\kappa_{0}\left(1, \underline{\omega}_{m}\right): \kappa_{0} \in \mathbb{Z}\right\} .
$$

The condition (1.34) can now be reexpressed as

$$
\pi_{\kappa} U_{\kappa}=U_{\kappa} \text { for all } \kappa \in \mathbb{Z} \times \mathbb{R}
$$

where

$$
\pi_{\kappa}=\left\{\begin{array}{l}
P_{m}, \text { if } \kappa \in \mathcal{C}_{m} \backslash 0 \\
I, \text { if } \kappa=0 \\
0, \text { if } \kappa \notin \mathcal{C}
\end{array} \quad, \text { for } P_{m}\right. \text { as in Lemma 1.11. }
$$

For $\mathcal{U}^{0}$ as in (1.32) if we now formally define the projector

$$
\mathbb{E} \mathcal{U}^{0}\left(x, \theta_{0}, \xi_{d}\right):=\sum_{\kappa=\left(\kappa_{0}, \kappa_{d}\right) \in \mathbb{Z} \times \mathbb{R}} \pi_{\kappa} U_{\kappa}(x) e^{i \kappa_{0} \theta_{0}+i \kappa_{d} \xi_{d}},
$$

we can rewrite the conditions (1.37) as

$$
\mathbb{E} \mathcal{U}^{0}=\mathcal{U}^{0}
$$

a condition that expresses both the polarization of Fourier components of $\mathcal{U}^{0}$ and the absence of noncharacteristic modes in the Fourier expansion. Whenever (1.40) is satisfied, note that 
(1.36) can be used to regroup the sum in (1.32) to recover the earlier expansion (1.24), where

$$
\underline{v}=U_{0} \text { and } \mathcal{V}_{m}^{0}\left(x, \theta_{0}+\underline{\omega}_{m} \xi_{d}\right)=\sum_{\kappa \in \mathcal{C}_{m} \backslash 0} U_{\kappa}(x) e^{i \kappa_{0} \theta_{0}+i \kappa_{d} \xi_{d}} .
$$

Later we shall use this flexibility to represent the approximate solution $u_{\epsilon}^{a}(x)$ either in the form (1.24) or in the form

$$
u_{\epsilon}^{a}(x)=\left.\mathcal{U}^{0}\left(x, \theta_{0}, \xi_{d}\right)\right|_{\theta_{0}=\frac{\phi_{0}}{\epsilon}, \xi_{d}=\frac{x_{d}}{\epsilon}} \text { where } \mathbb{E} \mathcal{U}^{0}=\mathcal{U}^{0} .
$$

Motivated again by the form of the singular system (1.26) and anticipating arguments in section 2.5 , we multiply through by $A_{d}^{-1}\left(\epsilon u_{\epsilon}\right)$ in (1.3) and write that system equivalently as

$$
\begin{aligned}
& \tilde{\mathcal{P}}\left(\epsilon u_{\epsilon}, \partial\right) u_{\epsilon}:= \\
& \quad \partial_{x_{d}} u_{\epsilon}+\sum_{j=0}^{d-1} \tilde{A}_{j}\left(\epsilon u_{\epsilon}\right) \partial_{x_{j}} u_{\epsilon}=A_{d}^{-1}\left(\epsilon u_{\epsilon}\right) \mathcal{F}\left(\epsilon u_{\epsilon}\right) u_{\epsilon}:=F\left(\epsilon u_{\epsilon}\right) u_{\epsilon}, \text { where } \tilde{A}_{j}:=A_{d}^{-1} A_{j} \\
& \left.B\left(\epsilon u_{\epsilon}\right) u_{\epsilon}\right|_{x_{d}=0}=G\left(x^{\prime}, \frac{x^{\prime} \cdot \beta}{\epsilon}\right) \\
& u_{\epsilon}=0 \text { in } x_{0}<0 .
\end{aligned}
$$

Notations 1.16. As in (1.43) we set $\tilde{A}_{j}=A_{d}^{-1} A_{j}$. Similarly, for $L\left(\partial_{x}\right)$ (resp. $\left.\mathcal{L}\left(\partial_{\theta_{0}}, \partial_{\xi_{d}}\right)\right)$ as in (1.12) (resp. (1.33)) we set

$$
\tilde{L}\left(\partial_{x}\right):=A_{d}(0)^{-1} L\left(\partial_{x}\right) \text { and } \tilde{\mathcal{L}}\left(\partial_{\theta_{0}}, \partial_{\xi_{d}}\right):=A_{d}(0)^{-1} \mathcal{L}\left(\partial_{\theta_{0}}, \partial_{\xi_{d}}\right) .
$$

The leading profile equations, that is, the transport equations that must be satisfied by the functions $\sigma_{m, k}\left(x, \theta_{m}\right)$ appearing in (1.24), arise as solvability conditions when one tries to improve the approximate solution $u_{\epsilon}^{a}(x)$ to a corrected approximate solution that we shall denote $u_{\epsilon}^{c}(x)$ :

$$
u_{\epsilon}^{c}(x):=\left.\left(\mathcal{U}^{0}\left(x, \theta_{0}, \xi_{d}\right)+\epsilon \mathcal{U}^{1}\left(x, \theta_{0}, \xi_{d}\right)\right)\right|_{\theta_{0}=\frac{\phi_{0}}{\epsilon}, \xi_{d}=\frac{x_{d}}{\epsilon}} .
$$

Here $\mathcal{U}^{1}$ is assumed to be of the form (1.32), but does not necessarily, and in fact will not, satisfy $\mathbb{E} \mathcal{U}^{1}=\mathcal{U}^{1}$. Plugging $u_{\epsilon}^{c}$ as in (1.42) into (1.43) we find that the terms of order $\epsilon^{0}$ cancel out if and only if

$$
\tilde{\mathcal{L}}\left(\partial_{\theta_{0}}, \partial_{\xi_{d}}\right) \mathcal{U}^{1}+\tilde{L}\left(\partial_{x}\right) \mathcal{U}^{0}+\mathcal{M}\left(\mathcal{U}^{0}\right) \partial_{\theta_{0}} \mathcal{U}^{0}=F(0) \mathcal{U}^{0},
$$

where

$$
\mathcal{M}\left(\mathcal{U}^{0}\right):=\sum_{j=0}^{d-1} \partial_{u} \tilde{A}_{j}(0) \mathcal{U}^{0} \beta_{j}
$$


Assuming that $\mathcal{H}\left(x, \theta_{0}, \xi_{d}\right)$ has an expansion like (1.32), the problem $\tilde{\mathcal{L}}\left(\partial_{\theta_{0}}, \partial_{\xi_{d}}\right) \mathcal{U}^{1}=\mathcal{H}$ is formally equivalent to the family of equations

$$
i \tilde{L}\left(\kappa_{0} \beta, \kappa_{d}\right) U_{\kappa}^{1}(x)=H_{\kappa}(x) \text { for all } \kappa \in \mathbb{Z} \times \mathbb{R} .
$$

Using Lemma (1.11) and considering the cases $\kappa=0, \kappa \in \mathcal{C} \backslash 0, \kappa \notin \mathcal{C}$, we see that the equations (1.48) are solvable if and only if

$$
\pi_{\kappa} H_{\kappa}=0 \text { for all } \kappa \in \mathbb{Z} \times \mathbb{R} \text {. }
$$

Equivalently, we have

Lemma 1.17. The equations (1.48) are solvable if and only if $\mathbb{E} \mathcal{H}=0$.

This completes our formal derivation of the leading profile equations for problems with fixed boundaries:

$$
\begin{aligned}
& \text { a) } \mathbb{E} \mathcal{U}^{0}=\mathcal{U}^{0} \\
& \text { b) } \mathbb{E}\left(\tilde{L}\left(\partial_{x}\right) \mathcal{U}^{0}+\mathcal{M}\left(\mathcal{U}^{0}\right) \partial_{\theta_{0}} \mathcal{U}^{0}\right)=\mathbb{E}\left(F(0) \mathcal{U}^{0}\right) \\
& \text { c) }\left.B(0) \mathcal{U}^{0}\right|_{x_{d}=0, \xi_{d}=0}=G\left(x^{\prime}, \theta_{0}\right) \\
& \text { d) } \mathcal{U}^{0}=0 \text { in } t<0 .
\end{aligned}
$$

We make rigorous sense of these equations in Proposition 2.2. There we show that when $s>\frac{d+1}{2}+1$, all terms appearing in (1.50) are well-defined when $\mathcal{U}^{0} \in \mathcal{P}_{T}^{s}$, the latter space being defined as the closure of the space of trigonometric polynomials in

$$
\mathcal{E}_{T}^{s}:=\left\{\mathcal{U}\left(x, \theta_{0}, \xi_{d}\right): \sup _{\xi_{d} \in \mathbb{R}}\left|\mathcal{U}\left(\cdot, \cdot, \xi_{d}\right)\right|_{E_{T}^{s}}<\infty\right\}
$$

for $E_{T}^{s}$ as in (1.9). The shock analogue of (1.50) involves the extra unknowns $\chi^{0}$ and $\chi^{1}$ and is given in (3.49).

Observe that the only nonlinear term in the profile equations (1.50) is the quadratic interaction term $\mathbb{E}\left(\mathcal{M}\left(\mathcal{U}^{0}\right) \partial_{\theta_{0}} \mathcal{U}^{0}\right)$, a vector whose components are sums of products involving just two functions.

The almost-periodic profile equations presented in [8], Theorem 2.3.5, for the initial value problem can be derived in a similar manner. With notation as in (1.6) the unknown in [8] is a function $\mathbb{V}\left(t, x^{\prime \prime}, \tau, \omega\right)$, periodic in $\omega$ but almost-periodic in $(\tau, \omega)$, where $\tau$ is now a placeholder for $\frac{t}{\epsilon}$. The equations have the same form as (1.50), except that the operators in place of $\tilde{L}\left(\partial_{x}\right)$ and $\mathcal{M}\left(\mathcal{U}^{0}\right) \partial_{\theta_{0}}$ are symmetric, conditions (c) and (d) are replaced by an initial condition at $t=0, \tau=0$, and in the definition of the operator $\mathbb{E}$ one has to use projectors corresponding to the roots $\underline{\tau}_{k}$ of $\operatorname{det} L(\tau, \alpha)=0$ instead of to the roots $\xi_{d}$ of $\operatorname{det} L\left(\beta, \xi_{d}\right)=0$.

In the context of [8], the operator acting on $\mathcal{U}^{0}$ in (1.50) (including $\mathbb{E}$ ) was symmetric hyperbolic on the space of functions satisfying $\mathbb{E} \mathcal{U}^{0}=\mathcal{U}^{0}$, and this allowed the authors of [8] 
to prove energy estimates for the linearized problem by working directly on the profiles $\mathcal{U}^{0}$, pairing the equation with $\mathcal{U}^{0}$ and integrating by parts. ${ }^{6}$ The matrix coefficients in our case are not symmetric, and even if they were, the boundary condition would force us to adopt a different approach. To treat the boundary terms that arise from integration by parts, we must separate incoming from outgoing modes, and this forces us to do a mode-by-mode analysis, where we reformulate (1.50) as a large system for the unknowns $\underline{v}(x), \sigma_{m, k}\left(x, \theta_{m}\right)$ appearing in the ansatz (1.24).

In order to derive the large system we reformulate the almost periodic profile equations in terms of periodic profiles $\mathcal{V}^{0}(x, \theta)$, where $\theta=\left(\theta_{1}, \ldots, \theta_{M}\right)$ and $\mathcal{V}^{0}$ has the form

$$
\mathcal{V}^{0}(x, \theta)=\underline{v}(x)+\sum_{m=1}^{M} \sum_{k=1}^{\nu_{k_{m}}} \sigma_{m, k}\left(x, \theta_{m}\right) r_{m, k} \quad(\text { recall }(1.24)) .
$$

The equations, given in (2.16) for the fixed boundary case and in (3.60) for the shock case, are expressed in terms of operators $\mathbb{E}^{\prime}(2.15)$ which, like the operators $\mathbb{E}$ in (1.50), are designed both to select characteristic modes (including resonances) and impose (via $P_{m}$ ) the polarization associated to those modes. Extra care is needed in defining $\mathbb{E}^{\prime}$ because, unlike the set of phases $\left\{\phi_{0}, x_{d}\right\}$, the set of phases $\left\{\phi_{m}, m=1, \ldots, M\right\}$ is not necessarily $\mathbb{Q}$-linearly independent. Because we are only concerned with quadratic interactions, the pairwise independence of the $\phi_{m}$ allows us to define $\mathbb{E}^{\prime}$ as a map on the spaces $H^{s ; 2}\left(\overline{\mathbb{R}}_{+}^{d+1} \times \mathbb{T}^{M}\right)$ (2.12) of $H^{s}$ functions periodic in $\theta$ whose Fourier series involve exponentials depending on at most two of the $\theta_{m}$. In the shock case, in order to handle shock front-interior interactions we must treat the boundary phase $\phi_{0}$ on an equal footing with the phases $\phi_{m}$, and so we define $\theta=\left(\theta_{0}, \theta_{1}, \ldots, \theta_{M}\right)^{7}$.

Once $\mathcal{V}^{0}$ is determined, the solution $\mathcal{U}^{0}$ to the almost periodic profile equations (1.50) is obtained by setting

$$
\mathcal{U}^{0}\left(x, \theta_{0}, \xi_{d}\right):=\mathcal{V}^{0}\left(x, \theta_{0}+\underline{\omega}_{1} \xi_{d}, \ldots, \theta_{0}+\underline{\omega}_{M} \xi_{d}\right) .
$$

The fact that for $s$ large enough solutions $\mathcal{V}^{0}(x, \theta) \in H_{T}^{s+1}(x, \theta)$ to $(2.16)$ yield solutions $\mathcal{U}^{0}\left(x, \theta_{0}, \xi_{d}\right) \in \mathcal{P}_{T}^{s}$ to (1.50) in this way is proved in Proposition 2.9. The main step is to establish a one-to-one correspondence between the resonances occurring in the two systems. The large system for individual modes is equivalent to (2.16) and is obtained from it by application of the projectors $\mathbb{E}_{0}^{\prime}, \mathbb{E}_{m, k}^{\prime}$ appearing in (2.47).

The large system in the fixed boundary case is a quasilinear, integro-differential system that couples an $N \times N$ system (2.63) in which all terms have nonzero mean, to a system of $N=\sum_{m=1}^{M} \nu_{k_{m}}$ scalar equations (2.64), where each line has zero mean. Additional coupling of the unknowns $\underline{v}(x), \sigma_{m, k}\left(x, \theta_{m}\right)$ occurs in the boundary equations (2.66). The integrals that appear in the interior equations are of two types. Each integral appearing in (2.63) or in (2.64), line (b), defines the mean of a product of two periodic functions. The integrals

\footnotetext{
${ }^{6}$ This feature of their profile equations permitted the authors of [8] to construct profiles even in situations where nonlinear interactions excite oscillations propagating in an infinite number of directions.

${ }^{7}$ Here we suppress \pm ; see Definition 3.8 .
} 
such as $I_{n_{q}, n_{p}, n_{r}}^{k, k^{\prime}}$ in (2.64), line (c), which are defined in (2.58), are interaction integrals that pick out resonances in the manner explained in Proposition 2.13. Resonances are generated in products like $\sigma_{p, k}\left(x, \frac{\phi_{p}}{\epsilon}\right) \partial_{\theta_{r}} \sigma_{r, k^{\prime}}\left(x, \frac{\phi_{r}}{\epsilon}\right)$ whenever there exists a relation of the form

$$
n_{q} \phi_{q}=n_{p} \phi_{p}+n_{r} \phi_{r}, \text { where } q \in\{1, \ldots, M\} \backslash\{p, r\} \text { and } n_{q}, n_{p}, n_{r} \in \mathbb{Z},
$$

in which case we refer to $\left(\phi_{q}, \phi_{p}, \phi_{r}\right)$ as a triple of resonant phases. This relation implies, for example, that $\phi_{p}$ oscillations interact with $\phi_{r}$ oscillations to produce $\phi_{q}$ oscillations. Of course, (1.54) also implies that $\phi_{q}$ oscillations interact with $\phi_{p}$ oscillations to produce $\phi_{r}$ oscillations, and so on. In the large system for shocks (3.68), (3.69), (3.70), interaction integrals of another type appear such as $S_{n_{0}, \underline{n}_{p}, n_{s}}^{k}$ in (3.69), line (d), which describe the creation of interior oscillations due to shock front-interior interactions and are associated to relations like

$$
n_{0} \phi_{0}=\underline{n}_{p} \phi_{p}+n_{s} \phi_{s} .
$$

Example 1.18 (Euler resonances). On the half space $\left\{\left(t, x_{1}, x_{2}\right): x_{2}>0\right\}$ consider the $2 D$ isentropic, compressible Euler equations in the variables $(v, u)$, where $v=\frac{1}{\rho}$ is specific volume and velocity $u=\left(u_{1}, u_{2}\right)$, linearized about a constant state $\left(v, 0, u_{2}\right)$ with $v>0$, $u_{2}<0,\left|u_{2}\right|<c$ (subsonic, outgoing). We have

$$
A_{0}=I, A_{1}=\left(\begin{array}{ccc}
0 & -v & 0 \\
-c^{2} / v & 0 & 0 \\
0 & 0 & 0
\end{array}\right), A_{2}=\left(\begin{array}{ccc}
u_{2} & 0 & -v \\
0 & u_{2} & 0 \\
-c^{2} / v & 0 & u_{2}
\end{array}\right)
$$

The hyperbolic region is $\mathcal{H}=\left\{(\tau, \eta) \in \mathbb{R}^{2}:|\tau|>\sqrt{c^{2}-u_{2}^{2}}|\eta|\right\}$, and we choose

$$
\phi_{0}\left(t, x_{1}\right)=t \underline{\tau}+x_{1} \underline{\eta}, \text { where } \underline{\eta}>0, \underline{\tau}=c \underline{\eta} .
$$

From (1.56) the corresponding interior phases are found to be $\phi_{m}\left(t, x_{1}, x_{2}\right)=\phi_{0}\left(t, x_{1}\right)+$ $\underline{\omega}_{m} x_{2}, m=1,2,3$, where

$$
\underline{\omega}_{1}=\frac{2 M}{1-M^{2}} \underline{\eta}, \quad \underline{\omega}_{2}=0, \quad \underline{\omega}_{3}=-\frac{1}{M} \underline{\eta}
$$

with $M:=\frac{u_{2}}{c} \in(-1,0)$ the Mach number. The only incoming phase is $\phi_{1}$.

To find resonances we look for $\left(n_{1}, n_{2}, n_{3}\right) \in \mathbb{Z}^{3} \backslash 0$ such that $n_{1} \phi_{1}=n_{2} \phi_{2}+n_{3} \phi_{3}$, that is,

$$
n_{1}\left(\begin{array}{l}
\frac{\tau}{\eta} \\
\underline{\underline{\omega}}
\end{array}\right)=n_{2}\left(\begin{array}{l}
\underline{\tau} \\
\underline{\eta} \\
\underline{\omega}_{2}
\end{array}\right)+n_{3}\left(\begin{array}{l}
\underline{\tau} \\
\underline{\eta} \\
\underline{\omega}_{3}
\end{array}\right) \Leftrightarrow\left\{\begin{array}{l}
n_{1}=n_{2}+n_{3} \\
n_{1} \underline{\omega}_{1}=n_{2} \underline{\omega}_{2}+n_{3} \underline{\omega}_{3}
\end{array} .\right.
$$

Thus, the phases are in resonance if and only if

$$
n_{2}=n_{1}-n_{3} \text { and } n_{1} \frac{2 M^{2}}{1-M^{2}}=-n_{3} .
$$


Since $M^{2} \in(0,1)$, we see that resonance occurs if and only if

$$
\frac{M^{2}}{1-M^{2}} \in\{q \in \mathbb{Q}: q>0\}
$$

a countable set of allowable values.

Triples of resonant phases for 3D Euler may be computed similarly.

The solution of the large system is completed in Proposition 2.21, and is based on the estimate of Proposition 2.19 for solutions to the iteration scheme (2.71),(2.72). The idea is to combine a Kreiss-type estimate for the nonzero mean system (2.71) with an estimate for the zero mean system (2.72) proved by taking the $L^{2}\left(d x d \theta_{p}\right)$-pairing with $\sigma_{p, l}^{n+1}\left(x, \theta_{p}\right)$ and integrating by parts. The integral terms in (2.72) are well-suited to such an estimate, since one can integrate by parts (before pairing) to pull $\theta$-derivatives off $(n+1)$-st iterates. However, sums like

$$
\sum_{j, k, k^{\prime}} b_{p, l, j}^{k, k^{\prime}} \sigma_{p, k}^{n}\left(x, \theta_{p}\right) \partial_{\theta_{p}} \sigma_{p, k^{\prime}}^{n+1}\left(x, \theta_{p}\right), \quad \text { where } b_{p, l, j}^{k, k^{\prime}}=\ell_{p, l} \cdot \beta_{j}\left(\partial_{u} \tilde{A}_{j}(0) r_{p, k}\right) r_{p, k^{\prime}}
$$

in the second line of $(2.72)$ (a) present a potentially serious obstacle to proving an $L^{2}$ estimate, since there is no obvious way to avoid having $\theta$-derivatives fall on $(n+1)$-st iterates after pairing with $\sigma_{p, l}^{n+1}\left(x, \theta_{p}\right)$. This problem does not arise when the operator $L\left(\partial_{x}\right)$ is strictly hyperbolic, since then the indices $k, k^{\prime}$, and $l$ are absent (i.e., they only take the value 1$)$, and after pairing with $\sigma_{p}^{n+1}\left(x, \theta_{p}\right)$ one can write

$$
\sigma_{p}^{n}\left(x, \theta_{p}\right) \partial_{\theta_{p}} \sigma_{p}^{n+1}\left(x, \theta_{p}\right) \sigma_{p}^{n+1}\left(x, \theta_{p}\right)=\frac{1}{2} \sigma_{p}^{n}\left(x, \theta_{p}\right) \partial_{\theta_{p}}\left(\sigma_{p}^{n+1}\left(x, \theta_{p}\right)\right)^{2},
$$

and then integrate by parts to move the derivative off the $(n+1)$-st iterate. The difficulty is overcome using Proposition 2.18, which implies that

$$
\sum_{j, k} b_{p, l, j}^{k, k^{\prime}} \sigma_{p, k}^{n}\left(x, \theta_{p}\right)=0 \text { unless } k^{\prime}=l .
$$

Using (1.64) one can now pair the sum in (1.62) with $\sigma_{p, l}^{n+1}\left(x, \theta_{p}\right)$ and do the estimate as in the strictly hyperbolic case. The proof of Proposition 2.18 is a variation on the argument used by Lax [10] to show that $\phi_{m}$ oscillations are transported with group velocity $\mathbf{v}_{m}$.

Finally, traces of outgoing modes $\sigma_{p, l}^{n+1}, p \in \mathcal{O}$ are readily estimated since they occur with a good sign after integration by parts; traces of incoming modes can then be estimated after using the uniform stability condition to express them in terms of $G^{*}$ and outgoing modes as in (2.67).

\subsection{Error analysis by simultaneous Picard iteration}

The iteration schemes for the singular system (1.8) and the profile equations (1.50) are written side by side in (2.104), (2.105). For $s$ large and some $T_{0}>0$ the proof of Theorem 
1.13 produces a sequence of iterates $U_{\epsilon}^{n}\left(x, \theta_{0}\right)$, bounded in $E_{T_{0}}^{s}$ uniformly with respect to $n$ and $\epsilon$, and such that

$$
\lim _{n \rightarrow \infty} U_{\epsilon}^{n}=U_{\epsilon} \text { in } E_{T_{0}}^{s-1} \text { uniformly with respect to } \epsilon \in\left(0, \epsilon_{0}\right],
$$

where $U_{\epsilon}$ is the solution of (1.8). On the other hand the construction of profiles in Propositions 2.19 and 2.21 yields a sequence of profile iterates $\mathcal{U}^{0, n}\left(x, \theta_{0}, \xi_{d}\right)$ bounded in $\mathcal{E}_{T_{0}}^{s}(1.51)$ and converging in $\mathcal{E}_{T_{0}}^{s-1}$ to a solution $\mathcal{U}^{0}$ of (1.50). By Lemma 2.25 this implies that the functions $\mathcal{U}_{\epsilon}^{0, n}\left(x, \theta_{0}\right):=\mathcal{U}^{0, n}\left(x, \theta_{0}, \frac{x_{d}}{\epsilon}\right)$ satisfy

$$
\lim _{n \rightarrow \infty} \mathcal{U}_{\epsilon}^{0, n}=\mathcal{U}_{\epsilon}^{0} \text { in } E_{T_{0}}^{s-1} \text { uniformly with respect to } \epsilon \in\left(0, \epsilon_{0}\right] .
$$

Thus, in order to conclude $\lim _{\epsilon \rightarrow 0} \mathcal{U}_{\epsilon}^{0}\left(x, \theta_{0}\right)=U_{\epsilon}\left(x, \theta_{0}\right)$ in $E_{T_{0}}^{s-1}$ and thereby complete the proof of Theorem 1.15, it would suffice to show:

$$
\text { for each } n, \lim _{\epsilon \rightarrow 0} \mathcal{U}_{\epsilon}^{0, n}=U_{\epsilon}^{n} \text { in } E_{T_{0}}^{s-1} \text {. }
$$

The statement (1.67) is proved by induction in section 2.5. It is natural to try to apply the estimate of Proposition 2.23 to the difference $\mathcal{U}_{\epsilon}^{0, n+1}-U_{\epsilon}^{n+1}$, but the problem is that for any given $n, \mathcal{U}_{\epsilon}^{0, n+1}$ does not by itself provide a very good approximate solution to the boundary problem (2.104) that defines $U_{\epsilon}^{n+1}$. Indeed, substitution of $\mathcal{U}_{\epsilon}^{0, n+1}$ into (2.104)(a) yields an $O(1)$ error, call it $R_{\epsilon}\left(x, \theta_{0}\right)$, in $E_{T_{0}}^{s-1}$. Since $\mathcal{U}^{0, n+1}$ satisfies $(2.105)$, the error satisfies

$$
R_{\epsilon}\left(x, \theta_{0}\right)=\mathcal{R}\left(x, \theta_{0}, \frac{x_{d}}{\epsilon}\right) \text { with } \mathbb{E} \mathcal{R}=0 .
$$

Recalling the corrected approximate solution (1.45), one might hope to solve away the error by using Lemma 1.17 to construct a corrector $\mathcal{U}^{1}\left(x, \theta_{0}, \xi_{d}\right) \in \mathcal{E}_{T_{0}}^{s-1}$ such that

$$
\tilde{\mathcal{L}}\left(\partial_{\theta_{0}}, \partial_{\xi_{d}}\right) \mathcal{U}^{1}=-\mathcal{R} .
$$

That is generally not possible, however, because of the small divisor problem discussed in Remark 2.26. We remedy this using an idea of [8], which is to approximate $\mathcal{U}^{0, n}$ and $\mathcal{U}^{0, n+1}$ by trigonometric polynomials and to construct a trigonometric polynomial corrector $\mathcal{U}_{p}^{1}$. For fixed $\delta>0$, after taking trigonometric polynomial approximations with error $<\delta$ in $\mathcal{E}_{T_{0}}^{s}$, we construct a corrector $\mathcal{U}_{p, \epsilon}^{1}$ which, though it does not solve away $R_{\epsilon}$, solves away "all but $O(\delta)$ " of $R_{\epsilon}$ in $E_{T_{0}}^{s-1}$ (see (2.129)). This then allows us to conclude

$$
\lim _{\epsilon \rightarrow 0} \mathcal{U}_{\epsilon}^{0, n+1}=U_{\epsilon}^{n+1} \text { in } E_{T_{0}}^{s-1}
$$

by an application of Proposition 2.23.

The error analysis is given in section 3.4 for the case of shocks, where the main new point is to incorporate the free boundary into the argument. The two iteration schemes are given in (3.89),(3.90), and now in place of (1.67) we show

$$
\text { for each } n, \lim _{\epsilon \rightarrow 0}\left|U_{\epsilon}^{n}-\mathcal{U}_{\epsilon}^{0, n}\right|_{E_{T_{0}}^{s-1}}=0 \text { and } \lim _{\epsilon \rightarrow 0}\left\langle\nabla^{\epsilon}\left(\chi_{\epsilon}^{n}-\left(\chi^{0, n}+\epsilon \chi^{1, n}\right)\right)\right\rangle_{H_{T_{0}}^{s}}=0 \text {, }
$$


where $\nabla^{\epsilon}:=\partial_{x^{\prime}}+\frac{\beta \partial_{\theta_{0}}}{\epsilon}$. Trigonometric polynomial approximations must now be taken for $\left(\mathcal{U}^{0, n}, \chi^{0, n}, \chi^{1, n}\right)$ as well as for the corresponding $(n+1)$-st iterates. It turns out that only an interior corrector $\mathcal{U}_{p}^{1}$, and no front corrector, is needed. The limits in (1.71) for the case $n+1$ are then deduced by an application of the estimate in Proposition 3.15.

Remark 1.19. In the case of fixed boundaries we do not assume that the original system (1.2) is expressible in conservative form, but in the case of shocks we only treat systems of conservation laws (3.1). Conservative form is used only in formulating the "boundary" condition, which is the usual Rankine-Hugoniot condition. It is clear that our results on shocks extend to the case of nonconservative shocks if one uses the notions of generalized Rankine-Hugoniot condition and uniform stability defined in [6].

\section{Fixed boundary problems}

Before studying the free boundary problem associated to multidimensional shocks, we first carry out the program outlined above in the easier case of fixed boundaries.

\subsection{Profile equations: formulation with almost-periodic profiles}

Let us recall the almost periodic profile equations derived above:

$$
\begin{aligned}
& \text { a) } \mathbb{E} \mathcal{U}^{0}=\mathcal{U}^{0} \\
& \text { b) } \mathbb{E}\left(\tilde{L}\left(\partial_{x}\right) \mathcal{U}^{0}+\mathcal{M}\left(\mathcal{U}^{0}\right) \partial_{\theta_{0}} \mathcal{U}^{0}\right)=\mathbb{E}\left(F(0) \mathcal{U}^{0}\right) \\
& \text { c) }\left.B(0) \mathcal{U}^{0}\right|_{x_{d}=0, \xi_{d}=0}=G\left(x^{\prime}, \theta_{0}\right) \\
& \text { d) } \mathcal{U}^{0}=0 \text { in } t<0 .
\end{aligned}
$$

To make rigorous sense of (2.1) we introduce the following spaces:

Definition 2.1. 1.) For $s \geq 0$ let $E_{T}^{s}:=\left\{U\left(x, \theta_{0}\right) \in C\left(x_{d}, H_{T}^{s}\left(x^{\prime}, \theta_{0}\right)\right) \cap L^{2}\left(x_{d}, H_{T}^{s+1}\left(x^{\prime}, \theta_{0}\right)\right)\right\}$ with the norm

$$
\left\|U\left(x, \theta_{0}\right)\right\|_{s, T}:=|U|_{\infty, s, T}+|U|_{0, s+1, T} .
$$

2.) Let $\mathcal{E}_{T}^{s}:=\left\{\mathcal{U}\left(x, \theta_{0}, \xi_{d}\right):\|\mathcal{U} \mid\|_{s, T}:=\sup _{\xi_{d} \in \mathbb{R}}\left\|\mathcal{U}\left(\cdot, \cdot, \xi_{d}\right)\right\|_{s, T}<\infty\right\}$.

3.) We define a trigonometric polynomial in $\mathcal{E}_{T}^{s}$ to be a finite sum of the form (1.32), where the coefficients $U_{\kappa}(x) \in C\left(x_{d}, H_{T}^{s}\left(x^{\prime}\right)\right) \cap L^{2}\left(x_{d}, H_{T}^{s+1}\left(x^{\prime}\right)\right)$.

4.) Let $\mathcal{P}_{T}^{s}$ denote the closure in $\mathcal{E}_{T}^{s}$ of the space of trigonometric polynomials.

The following Proposition shows when $s>\frac{d+1}{2}+1$ all the terms appearing in the profile equations (2.1) are well-defined when $\mathcal{U}^{0} \in \mathcal{P}_{T}^{s}$.

Proposition 2.2. a) For $s>(d+1) / 2$ the spaces $E_{T}^{s}, \mathcal{E}_{T}^{s}$ and $\mathcal{P}_{T}^{s}$ are Banach algebras.

b) The projector $\mathbb{E}$ has a unique continuous extension from the subspace of trigonometric polynomials in $\mathcal{E}_{T}^{0}$ to an operator $\mathbb{E}: \mathcal{P}_{T}^{0} \rightarrow \mathcal{P}_{T}^{0}$. By restriction $\mathbb{E}$ maps $\mathcal{P}_{T}^{s} \rightarrow \mathcal{P}_{T}^{s}$ for $s \geq 0$. The norms of these operators are bounded uniformly with respect to $T$ for $0 \leq T \leq T_{0}$. 
Proof. Part a) is a consequence of Sobolev embedding and the fact that $L^{\infty}\left(\Omega_{T}\right) \cap H^{s}\left(b \Omega_{T}\right)$ is a Banach algebra for $s \geq 0$.

The proof of part b) is similar to the proof of Proposition 6.2.1 of [8]. Given a finite sum $\mathcal{U}$ of the form (1.32) set

$$
\mathcal{U}_{\kappa_{0}}\left(x, \xi_{d}\right)=\sum_{\kappa_{d}} U_{\kappa_{0}, \kappa_{d}}(x) e^{i \kappa_{d} \xi_{d}}
$$

Thus,

$$
(\mathbb{E} \mathcal{U})_{\kappa_{0}}\left(x, \xi_{d}\right)=\sum_{\left\{\kappa_{d}:\left(\kappa_{0}, \kappa_{d}\right) \in \mathcal{C}\right\}} \pi_{\kappa_{0}, \kappa_{d}} U_{\kappa_{0}, \kappa_{d}}(x) e^{i \kappa_{d} \xi_{d}},
$$

from which we obtain

$$
\left|(\mathbb{E} \mathcal{U})_{\kappa_{0}}\left(x, \xi_{d}\right)\right|^{2} \leq C M \sum_{\left\{\kappa_{d}:\left(\kappa_{0}, \kappa_{d}\right) \in \mathcal{C}\right\}}\left|U_{\kappa_{0}, \kappa_{d}}(x)\right|^{2},
$$

Now from (2.2) we have

$$
U_{\kappa_{0}, \kappa_{d}}(x)=\lim _{R \rightarrow \infty} R^{-1} \int_{0}^{R} \mathcal{U}_{\kappa_{0}}(x, s) e^{-i \kappa_{d} s} d s,
$$

which implies

$$
\left|U_{\kappa_{0}, \kappa_{d}}(x)\right|^{2}=\lim _{R \rightarrow \infty} R^{-2}\left|\int_{0}^{R} \mathcal{U}_{\kappa_{0}}(x, s) e^{-i \kappa_{d} s} d s\right|^{2} \leq \liminf _{R \rightarrow \infty} R^{-1} \int_{0}^{R}\left|\mathcal{U}_{\kappa_{o}}(x, s)\right|^{2} d s .
$$

In view of (2.5) this implies

$$
\left|(\mathbb{E} \mathcal{U})_{\kappa_{0}}\left(x, \xi_{d}\right)\right|^{2} \leq C M^{2} \liminf _{R \rightarrow \infty} R^{-1} \int_{0}^{R}\left|\mathcal{U}_{\kappa_{o}}(x, s)\right|^{2} d s .
$$

Applying Fatou's Lemma to $\sum_{\kappa_{0}} \int_{x^{\prime}}$ we find

$$
\begin{aligned}
& \sum_{\kappa_{0}}\left|(\mathbb{E} \mathcal{U})_{\kappa_{0}}\left(x^{\prime}, x_{d}, \xi_{d}\right)\right|_{L^{2}\left(x^{\prime}\right)}^{2} \leq C M^{2} \liminf _{R \rightarrow \infty} R^{-1} \int_{0}^{R} \sum_{\kappa_{0}}\left|\mathcal{U}_{\kappa_{0}}\left(x^{\prime}, x_{d}, s\right)\right|_{L^{2}\left(x^{\prime}\right)}^{2} d s \\
& =C M^{2} \liminf _{R \rightarrow \infty} R^{-1} \int_{0}^{R}\left|\mathcal{U}\left(x^{\prime}, x_{d}, \theta_{0}, s\right)\right|_{L^{2}\left(x^{\prime}, \theta_{0}\right)}^{2} d s \leq C M^{2} \sup _{s}\left|\mathcal{U}\left(x^{\prime}, x_{d}, \theta_{0}, s\right)\right|_{L^{2}\left(x^{\prime}, \theta_{0}\right)}^{2} .
\end{aligned}
$$

After similarly applying Fatou's Lemma to $\sum_{\kappa_{0}} \int_{x}$ and summing, we find $|\mathbb{E} \mathcal{U}|_{\mathcal{E}_{T}^{0}} \leq \sqrt{C} M|\mathcal{U}|_{\mathcal{E}_{T}^{0}}$, and so $\mathbb{E}$ extends continuously from $\mathcal{P}_{T}^{0}$ to itself. The result for $\mathcal{P}_{T}^{s}$ follows readily by commuting through with $\partial_{x^{\prime}, \theta_{0}}^{\alpha}$.

Remark 2.3. The quadratic term $\mathcal{M}\left(\mathcal{U}^{0}\right) \partial_{\theta_{0}} \mathcal{U}^{0}$ in (2.1)(b) describes the nonlinear interactions that influence the leading profile $\mathcal{U}^{0}$. It is important for the error analysis in section 2.5 that, as a result of our taking $\tilde{A}_{d}\left(\epsilon u_{\epsilon}\right)=I$, the term $\partial_{u} \tilde{A}_{d}(0) \mathcal{U}^{0} \partial_{\xi_{d}} \mathcal{U}^{0}$ in (1.46) vanishes. 


\subsection{Profile equations: reformulation with periodic profiles}

Next we derive the periodic form of the profile equations that we use for the construction of profiles.

Functions $\mathcal{V}(x, \theta) \in L^{2}\left(\overline{\mathbb{R}}_{+}^{d+1} \times \mathbb{T}^{M}\right)$ have Fourier series

$$
\mathcal{V}(x, \theta)=\sum_{\alpha \in \mathbb{Z}^{M}} V_{\alpha}(x) e^{i \alpha \cdot \theta} .
$$

Since only quadratic interactions are important in the problems studied here, for $k=1,2$ we let

$$
\mathbb{Z}^{M ; k}=\left\{\alpha \in \mathbb{Z}^{M}: \text { at most } k \text { components of } \alpha \text { are nonzero }\right\},
$$

and we consider the subspace $H^{s ; k}\left(\overline{\mathbb{R}}_{+}^{d+1} \times \mathbb{T}^{M}\right) \subset H^{s}\left(\overline{\mathbb{R}}_{+}^{d+1} \times \mathbb{T}^{M}\right)$ defined by

$$
H^{s ; k}\left(\overline{\mathbb{R}}_{+}^{d+1} \times \mathbb{T}^{M}\right)=\left\{\mathcal{V}(x, \theta) \in H^{s}\left(\overline{\mathbb{R}}_{+}^{d+1} \times \mathbb{T}^{M}\right): \mathcal{V}(x, \theta)=\sum_{\alpha \in \mathbb{Z}^{M ; k}} V_{\alpha}(x) e^{i \alpha \cdot \theta}\right\}
$$

Clearly, multiplication defines a continous map

$$
H^{s ; 1}\left(\overline{\mathbb{R}}_{+}^{d+1} \times \mathbb{T}^{M}\right) \times H^{s ; 1}\left(\overline{\mathbb{R}}_{+}^{d+1} \times \mathbb{T}^{M}\right) \rightarrow H^{s ; 2}\left(\overline{\mathbb{R}}_{+}^{d+1} \times \mathbb{T}^{M}\right)
$$

for $s>(d+1+2) / 2$.

Definition 2.4. Setting $\phi:=\left(\phi_{1}, \ldots, \phi_{M}\right)$, we say $\alpha \in \mathbb{Z}^{M ; 2}$ is a characteristic mode and write $\alpha \in \mathcal{C}^{\prime}$ if $\operatorname{det} L(d(\alpha \cdot \phi))=0$. We decompose

$$
\mathcal{C}^{\prime}=\cup_{m=1}^{M} \mathcal{C}_{m}^{\prime} \text {, where } \mathcal{C}_{m}^{\prime}=\left\{\alpha \in \mathbb{Z}^{M ; 2}: \alpha \cdot \phi=n_{\alpha} \phi_{m} \text { for some } n_{\alpha} \in \mathbb{Z}\right\} .
$$

Since $\phi_{i}$ and $\phi_{j}$ are linearly independent for $i \neq j$, any $\alpha \in \mathbb{Z}^{M ; 2} \backslash 0$ belongs to at most one of the sets $\mathcal{C}_{m}^{\prime}$. We define the projector $\mathbb{E}^{\prime}: H^{s ; 2}\left(\overline{\mathbb{R}}_{+}^{d+1} \times \mathbb{T}^{M}\right) \rightarrow H^{s ; 1}\left(\overline{\mathbb{R}}_{+}^{d+1} \times \mathbb{T}^{M}\right)$ by

$$
\mathbb{E}^{\prime}=\mathbb{E}_{0}^{\prime}+\sum_{m=1}^{M} \mathbb{E}_{m}^{\prime}, \text { where } \mathbb{E}_{0}^{\prime} \mathcal{V}=V_{0} \text { and } \mathbb{E}_{m}^{\prime} \mathcal{V}=\sum_{\alpha \in \mathcal{C}_{m}^{\prime} \backslash 0} P_{m} V_{\alpha}(x) e^{i n_{\alpha} \theta_{m}}
$$

Remark 2.5. To see that $\mathbb{E}^{\prime}$ has the stated mapping property note that for each $n \in \mathbb{Z}$, the number of $\alpha \in \mathcal{C}_{m}^{\prime} \backslash 0$ such that $n_{\alpha}=n$ is bounded by a number $M$ that can be chosen independent of $n$. Also, for every $\alpha \in \mathcal{C}_{m}^{\prime} \backslash 0$ we have $\left|n_{\alpha}\right| \leq C|\alpha|$, for a $C$ that can be chosen independent of $\alpha$. See Remark 2.10 for a related discussion of triples of resonant phases. 
Consider next the following set of equations for $\mathcal{V}^{0}(x, \theta) \in H^{s ; 2}\left(\overline{\mathbb{R}}_{+}^{d+1} \times \mathbb{T}^{M}\right)$ for $s$ sufficiently large (and specified later):

$$
\begin{aligned}
& \text { a) } \mathbb{E}^{\prime} \mathcal{V}^{0}=\mathcal{V}^{0} \\
& \text { b) } \mathbb{E}^{\prime}\left(\tilde{L}\left(\partial_{x}\right) \mathcal{V}^{0}+\mathcal{M}^{\prime}\left(\mathcal{V}^{0}\right) \partial_{\theta} \mathcal{V}^{0}\right)=\mathbb{E}^{\prime}\left(F(0) \mathcal{V}^{0}\right) \text { in } x_{d} \geq 0 \\
& \text { c) } B(0) \mathcal{V}^{0}\left(x^{\prime}, 0, \theta_{0}, \ldots, \theta_{0}\right)=G\left(x^{\prime}, \theta_{0}\right) \\
& \text { d) } \mathcal{V}^{0}=0 \text { in } t<0 .
\end{aligned}
$$

Here

$$
\mathcal{M}^{\prime}\left(\mathcal{V}^{0}\right) \partial_{\theta} \mathcal{V}^{0}:=\sum_{m=1}^{M} \mathcal{M}_{m}^{\prime}\left(\mathcal{V}^{0}\right) \partial_{\theta_{m}} \mathcal{V}^{0}, \text { with } \mathcal{M}_{m}^{\prime}\left(\mathcal{V}^{0}\right)=\sum_{j=0}^{d-1} \partial_{u} \tilde{A}_{j}(0) \mathcal{V}^{0} \frac{\partial \phi_{m}}{\partial x_{j}}
$$

Note that since $\partial_{x_{j}} \phi_{m}=\beta_{j}$ for all $m$, we have $\mathcal{M}_{m}^{\prime}\left(\mathcal{V}^{0}\right)=\mathcal{M}\left(\mathcal{V}^{0}\right)$ for $\mathcal{M}$ as defined in (1.47). Setting $\mathcal{L}^{\prime}\left(\partial_{\theta}\right)=\sum_{m=1}^{M} \tilde{L}\left(d \phi_{m}\right) \partial_{\theta_{m}}$, we observe that for $\mathcal{V}^{0} \in H^{s ; 2}\left(\overline{\mathbb{R}}_{+}^{d+1} \times \mathbb{T}^{M}\right)$,

$$
\mathbb{E}^{\prime} \mathcal{V}^{0}=\mathcal{V}^{0} \text { if and only if } \mathcal{V}^{0} \in H^{s ; 1}\left(\overline{\mathbb{R}}_{+}^{d+1} \times \mathbb{T}^{M}\right) \text { and } \mathcal{L}^{\prime}\left(\partial_{\theta}\right) \mathcal{V}^{0}=0,
$$

and (2.18) in turn is equivalent to the property that $\mathcal{V}^{0}$ has an expansion of the form

$$
\mathcal{V}^{0}=\underline{v}(x)+\sum_{m=1}^{M} \sum_{k=1}^{\nu_{k_{m}}} \sigma_{m, k}\left(x, \theta_{m}\right) r_{m, k}:=\underline{v}(x)+\sum_{m=1}^{M} \mathcal{V}_{m}^{0}\left(x, \theta_{m}\right)
$$

for some real-valued functions $\sigma_{m, k}$. Our strategy then will be to obtain a solution $\mathcal{U}^{0}\left(x, \theta_{0}, \xi_{d}\right)$ of (2.1) by first solving (2.16) for $\mathcal{V}^{0}(x, \theta) \in H_{T}^{s ; 1}(x, \theta), s$ sufficiently large, and then taking

$$
\mathcal{U}^{0}\left(x, \theta_{0}, \xi_{d}\right):=\mathcal{V}^{0}\left(x, \theta_{0}+\underline{\omega}_{1} \xi_{d}, \ldots, \theta_{0}+\underline{\omega}_{M} \xi_{d}\right) .
$$

Remark 2.6. The derivation of the profile systems (2.1) and (2.16) was based on the system (1.43) for $u_{\epsilon}$ in which the coefficients $\tilde{A}_{j}\left(\epsilon u_{\epsilon}\right)$ appear, rather than the original system (1.3) in which the coefficients $A_{j}\left(\epsilon u_{\epsilon}\right)$ appear. If the system (1.3) is used instead, one obtains a system of profile equations for $\mathcal{V}^{0}$ that is identical to (2.16), except that (2.16)(b) must be replaced by

$$
\mathbb{F}^{\prime}\left(L(\partial) \mathcal{V}^{0}+\mathcal{N}^{\prime}\left(\mathcal{V}^{0}\right) \partial_{\theta} \mathcal{V}^{0}\right)=\mathbb{F}^{\prime}\left(A_{d}(0) F(0) \mathcal{V}^{0}\right) \text { in } x_{d} \geq 0
$$

where

$$
\mathcal{N}^{\prime}\left(\mathcal{V}^{0}\right) \partial_{\theta} \mathcal{V}^{0}:=\sum_{m=1}^{M} \mathcal{N}_{m}^{\prime}\left(\mathcal{V}^{0}\right) \partial_{\theta_{m}} \mathcal{V}^{0}, \text { with } \mathcal{N}_{m}^{\prime}\left(\mathcal{V}^{0}\right)=\sum_{j=1}^{d} \partial_{u} A_{j}(0) \mathcal{V}^{0} \frac{\partial \phi_{m}}{\partial x_{j}},
$$

and the projector $\mathbb{F}^{\prime}$ is defined just like $\mathbb{E}^{\prime}(2.15)$, except that $P_{m}$ is replaced by $Q_{m}$, the projection onto $A_{d}(0) \operatorname{Ker} L\left(d \phi_{m}\right)$ in the decomposition

$$
\mathbb{C}^{N}=\oplus_{m=1}^{M} A_{d}(0) \operatorname{Ker} L\left(d \phi_{m}\right) .
$$


It is perhaps not clear, especially since the definition of the matrix $\mathcal{M}^{\prime}\left(\mathcal{V}^{0}\right)$ involves the derivatives $\partial_{u} \tilde{A}_{j}(0)$, that the profile system with $(2.21)$ in place of $(2.16)$ (b) is equivalent to the system (2.16), even though (1.43) is clearly equivalent to (1.3). The equivalence of the two profile systems can be verified directly using the following observations:

$$
\begin{aligned}
& \text { (a) } \partial_{u} \tilde{A}_{j}(0) w=A_{d}^{-1}(0)\left(\partial_{u} A_{j}(0) w\right)-A_{d}^{-1}(0)\left(\partial_{u} A_{d}(0) w\right) A_{d}^{-1}(0) A_{j}(0) \\
& \text { (b) } Q_{m} w=\sum_{k}\left(\ell_{m, k} \cdot A_{d}^{-1}(0) w\right) A_{d}(0) r_{m, k} \quad\left(\text { for } \ell_{m, k} \text { as in }(2.43)\right) .
\end{aligned}
$$

To carry out the strategy of obtaining solutions $\mathcal{U}^{0}$ of (2.1) from solutions $\mathcal{V}^{0}$ of (2.16), we first need to clarify the relation between the spaces $H_{T}^{s}\left(x, \theta_{j}\right)$ and the spaces $\mathcal{E}_{T}^{s}, \mathcal{P}_{T}^{s}$.

Lemma 2.7. For $s \in \mathbb{N}$ suppose $f\left(x, \theta_{j}\right) \in C\left(x_{d}, H_{T}^{s}\left(x^{\prime}, \theta_{j}\right)\right) \cap L^{2}\left(x_{d}, H_{T}^{s+1}\left(x^{\prime}, \theta_{j}\right)\right)$ and that its Fourier series converges in $H_{T}^{s}\left(x^{\prime}, \theta_{j}\right)$ uniformly with respect to $x_{d} \geq 0$. Then $\tilde{f}\left(x, \theta_{0}, \xi_{d}\right):=f\left(x, \theta_{0}+\underline{\omega}_{j} \xi_{d}\right) \in \mathcal{P}_{T}^{s}$. In particular, this conclusion holds if $f\left(x, \theta_{j}\right) \in$ $H_{T}^{s+1}\left(x, \theta_{j}\right)$ and we then have

$$
|\tilde{f}|_{\mathcal{E}_{T}^{s}} \leq C|f|_{H_{T}^{s+1}}
$$

Proof. The function $f\left(x, \theta_{j}\right)$ has a Fourier series

$$
f\left(x, \theta_{j}\right)=\sum_{n \in \mathbb{Z}} f_{n}(x) e^{i n \theta_{j}}
$$

whose partial sums are bounded and converge in $H_{T}^{s}\left(x^{\prime}, \theta_{j}\right)$ uniformly with respect to $x_{d} \geq 0$. It suffices to show that the partial sums of $\tilde{f}\left(x, \theta_{0}, \xi_{d}\right)$ are bounded and converge in $H_{T}^{s}\left(x^{\prime}, \theta_{0}\right)$ uniformly with respect to $\left(x_{d}, \xi_{d}\right)$, and are bounded and converge in $L^{2}\left(x_{d}, H_{T}^{s+1}\left(x^{\prime}, \theta_{0}\right)\right)$ uniformly with respect to $\xi_{d}$. Since

$$
\left|f\left(x, \theta_{j}\right)\right|_{H_{T}^{s}\left(x^{\prime}, \theta_{j}\right)}^{2}=\left.\sum_{n \in \mathbb{Z}|\beta| \leq s} \sum_{\mid x^{\prime}} \partial_{n}^{\beta} f_{n}(x)\right|_{L^{2}\left(x^{\prime}\right)} ^{2}(1+|n|)^{2(s-|\beta|)},
$$

it follows that for any integers $M_{1}<M_{2}$

$$
\left|\sum_{n=M_{1}}^{M_{2}} f_{n}(x) e^{i n\left(\theta_{0}+\underline{\omega}_{j} \xi_{d}\right)}\right|_{H_{T}^{s}\left(x^{\prime}, \theta_{0}\right)}^{2}=\left|\sum_{n=M_{1}}^{M_{2}} f_{n}(x) e^{i n \theta_{0}}\right|_{H_{T}^{s}\left(x^{\prime}, \theta_{0}\right)}^{2} .
$$

Thus, the partial sums of $\tilde{f}\left(x, \theta_{0}, \xi_{d}\right)$ are bounded and converge in $H_{T}^{s}\left(x^{\prime}, \theta_{0}\right)$ uniformly with respect to $\left(x_{d}, \xi_{d}\right)$. The condition involving $L^{2}\left(x_{d}, H_{T}^{s+1}\left(x^{\prime}, \theta_{0}\right)\right)$ is proved by integrating (2.28) (with $s+1$ in place of $s$ ) with respect to $x_{d}$ and observing that the left side is independent of $\xi_{d}$. The final statement follows from (2.28) and Sobolev embedding since

$$
L^{2}\left(x_{d}, H_{T}^{s+1}\left(x^{\prime}, \theta_{j}\right)\right) \cap H^{1}\left(x_{d}, H_{T}^{s}\left(x^{\prime}, \theta_{j}\right)\right) \subset H_{T}^{s+1}\left(x, \theta_{j}\right) .
$$


Remark 2.8. It follows from Lemma 2.7 that if $f\left(x, \theta_{j}\right) \in H_{T}^{s+2}\left(x, \theta_{j}\right)$, then $\tilde{f}\left(x, \theta_{0}, \xi_{d}\right) \in$ $\mathcal{P}_{T}^{s+1}$ and $\partial_{x_{d}} \tilde{f} \in \mathcal{P}_{T}^{s}$. Moreover, given $\delta>0$ there exists a trigonometric polynomial $\tilde{f}_{p}$ of the form (1.32) such that simultaneously

$$
\left|\tilde{f}-\tilde{f}_{p}\right|_{\mathcal{E}_{T}^{s+1}}<\delta \text { and }\left|\partial_{x_{d}} \tilde{f}-\partial_{x_{d}} \tilde{f}_{p}\right|_{\mathcal{E}_{T}^{s}}<\delta .
$$

The next Proposition justifies the strategy described above.

Proposition 2.9. Suppose $s \in \mathbb{N}, s>\frac{d+1}{2}+1$ and that $\mathcal{V}^{0}(x, \theta) \in H_{T}^{s+1}(x, \theta)$ satisfies the profile equations (2.16). Then

$$
\mathcal{U}^{0}\left(x, \theta_{0}, \xi_{d}\right):=\mathcal{V}^{0}\left(x, \theta_{0}+\underline{\omega}_{1} \xi_{d}, \ldots, \theta_{0}+\underline{\omega}_{M} \xi_{d}\right) \in \mathcal{P}_{T}^{s}
$$

satisfies the profile equations (2.1).

Proof. 1. Conditions (2.16)(c) and (d) for $\mathcal{V}^{0}$ immediately imply the corresponding conditions for $\mathcal{U}^{0}$.

2. The condition $\mathbb{E}^{\prime} \mathcal{V}^{0}=\mathcal{V}^{0}$ implies $\mathcal{V}^{0}$ has an expansion of the form (2.19), where $\underline{v}(x) \in$ $H_{T}^{s+1}(x)$ and $\sigma_{m, k}\left(x, \theta_{m}\right) \in H_{T}^{s+1}\left(x, \theta_{m}\right)$ for all $(m, k)$. Lemma $2.7 \operatorname{implies} \mathcal{U}^{0}\left(x, \theta_{0}, \xi_{d}\right) \in$ $\mathcal{P}_{T}^{s}$, so $\mathbb{E} \mathcal{U}^{0}$ is well-defined. We have

$$
\mathbb{E}\left(\sigma_{m, k}\left(x, \theta_{0}+\underline{\omega}_{m} \xi_{d}\right) r_{m, k}\right)=\sigma_{m, k}\left(x, \theta_{0}+\underline{\omega}_{m} \xi_{d}\right) r_{m, k}
$$

so $\mathbb{E} \mathcal{U}^{0}=\mathcal{U}^{0}$. Similarly, we obtain

$$
\mathbb{E}^{\prime}\left(\tilde{L}(\partial) \mathcal{V}^{0}\right)\left(x, \theta_{0}+\underline{\omega}_{1} \xi_{d}, \ldots, \theta_{0}+\underline{\omega}_{M} \xi_{d}\right)=\mathbb{E}\left(\tilde{L}(\partial) \mathcal{U}^{0}\right)\left(x, \theta_{0}, \xi_{d}\right)
$$

and the analogous statement for $\mathbb{E}^{\prime}\left(F(0) \mathcal{V}^{0}\right)$.

3. It remains only to check the interaction term. We have $\partial_{\theta_{m}} \sigma_{m, k}\left(x, \theta_{m}\right) \in H_{T}^{s}\left(x, \theta_{m}\right)$, so Lemma 2.7 implies

$$
\partial_{\theta_{m}} \sigma_{m, k}\left(x, \theta_{0}+\underline{\omega}_{m} \xi_{d}\right) r_{m, k} \in \mathcal{P}_{T}^{s-1}
$$

which is a Banach algebra by Proposition 2.2. Thus, $\mathcal{M}\left(\mathcal{U}^{0}\right) \partial_{\theta_{0}} \mathcal{U}^{0} \in \mathcal{P}_{T}^{s-1}$ and $\mathbb{E}\left(\mathcal{M}\left(\mathcal{U}^{0}\right) \partial_{\theta_{0}} \mathcal{U}^{0}\right)$ is well-defined. The term $\mathcal{M}\left(\mathcal{U}^{0}\right) \partial_{\theta_{0}} \mathcal{U}_{0}$ involves products like $\sigma_{m, k} \partial_{\theta_{p}} \sigma_{p, l}$, which in turn involves products like

$$
e^{i n\left(\theta_{0}+\underline{\omega}_{m} \xi_{d}\right)} e^{i n^{\prime}\left(\theta_{0}+\underline{\omega}_{p} \xi_{d}\right)}=e^{i\left(\left(n+n^{\prime}\right) \theta_{0}+\left(n \underline{\omega}_{m}+n^{\prime} \underline{\omega}_{p}\right) \xi_{d}\right)}, \text { where } n, n^{\prime} \in \mathbb{Z} .
$$

Whenever $n \underline{\omega}_{m}+n^{\prime} \underline{\omega}_{p}=\left(n+n^{\prime}\right) \underline{\omega}_{q}$ for some $q \in\{1, \cdots, M\} \backslash\{m, p\}$, we say that a resonance occurs in $\mathcal{M}\left(\mathcal{U}^{0}\right) \partial_{\theta_{0}} \mathcal{U}^{0}$ since then $\left(n+n^{\prime}\right)\left(1, \underline{\omega}_{q}\right) \in \mathcal{C}_{q}$. Similarly, we say that a resonance occurs in $\mathcal{M}^{\prime}\left(\mathcal{V}^{0}\right) \partial_{\theta} \mathcal{V}^{0}$ whenever a product $e^{i n \theta_{m}} e^{i n^{\prime} \theta_{p}}$ appears with $n \phi_{m}+n^{\prime} \phi_{p}=$ $\left(n+n^{\prime}\right) \phi_{q}$ for some $q$ as above, since then

$$
\left.\left(0, \ldots, n, 0, \ldots, 0, n^{\prime}, 0, \ldots, 0\right) \in \mathcal{C}_{q}^{\prime} \subset \mathbb{Z}^{M ; 2} \text { ( } n \text { in } m \text { th slot, } n^{\prime} \text { in } p \text { th slot }\right) .
$$


Clearly,

$$
n \underline{\omega}_{m}+n^{\prime} \underline{\omega}_{p}=\left(n+n^{\prime}\right) \underline{\omega}_{q} \text { if and only if } n \phi_{m}+n^{\prime} \phi_{p}=\left(n+n^{\prime}\right) \phi_{q},
$$

so there is a one-to-one correspondence between the two kinds of resonances. Obvious correspondences also hold for nonresonant products and products where $m=p$. In view of the definitions of $\mathbb{E}$ and $\mathbb{E}^{\prime}$ and the fact that $\mathcal{M}_{m}^{\prime}\left(\mathcal{V}^{0}\right)=\mathcal{M}\left(\mathcal{V}^{0}\right)$ for all $m$, it follows that for all $m, p, k, l$ :

$$
\begin{aligned}
& \mathbb{E}\left(\mathcal{M}\left(\sigma_{m, k} r_{m, k}\right) \partial_{\theta_{0}} \sigma_{p, l} r_{p, l}\right)\left(x, \theta_{0}, \xi_{d}\right)= \\
& \mathbb{E}^{\prime}\left(\mathcal{M}_{p}^{\prime}\left(\sigma_{m, k} r_{m, k}\right) \partial_{\theta_{p}} \sigma_{p, l} r_{p, l}\right)\left(x, \theta_{0}+\underline{\omega}_{1} \xi_{d}, \ldots, \theta_{0}+\underline{\omega}_{M} \xi_{d}\right) .
\end{aligned}
$$

Incorporating also the products involving $\underline{v}(x)$ we conclude

$$
\mathbb{E}\left(\mathcal{M}\left(\mathcal{U}^{0}\right) \partial_{\theta_{0}} \mathcal{U}^{0}\right)\left(x, \theta_{0}, \xi_{d}\right)=\mathbb{E}^{\prime}\left(\mathcal{M}^{\prime}\left(\mathcal{V}^{0}\right) \partial_{\theta} \mathcal{V}^{0}\right)\left(x, \theta_{0}+\underline{\omega}_{1} \xi_{d}, \ldots, \theta_{0}+\underline{\omega}_{M} \xi_{d}\right) .
$$

Remark 2.10. 1. Resonances occur in $\mathcal{M}^{\prime}\left(\mathcal{V}^{0}\right) \partial_{\theta} \mathcal{V}^{0}$ for each separate relation of the form

$$
n_{q} \phi_{q}=n_{p} \phi_{p}+n_{r} \phi_{r}, \text { where } q \in\{1, \ldots, M\} \backslash\{p, r\} \text { and } n_{q}, n_{p}, n_{r} \in \mathbb{Z},
$$

in which case we will refer to $\left(\phi_{q}, \phi_{p}, \phi_{r}\right)$ as a triple of resonant phases. This relation implies, for example, that $\phi_{p}$ oscillations interact with $\phi_{r}$ oscillations to produce $\phi_{q}$ oscillations. In listing triples like $\left(\phi_{q}, \phi_{p}, \phi_{r}\right)$ here and henceforth we use the ordering $q<p<r$, and we normalize so that $n_{q}>0$ and the greatest common divisor of the coefficients $\operatorname{gcd}\left(n_{q}, n_{p}, n_{r}\right)=1$. Since $d \phi_{p}$ and $d \phi_{r}$ are $\mathbb{Q}$ - (and $\mathbb{R}$-) linearly independent, this normalization uniquely determines $n_{q}, n_{p}, n_{r}$ for any ordered triple $\left(\phi_{q}, \phi_{p}, \phi_{r}\right)$ of resonant phases. There may be no, and there are clearly at most finitely many, triples of resonant phases. A given phase $\phi_{j}$ can belong to more than one ordered triple of resonant phases.

2. Later when studying the case of shocks, we will also need to consider relations of the form

$$
n_{0} \phi_{0}=n_{p} \phi_{p}+n_{q} \phi_{q}, \text { where } n_{0}, n_{p}, n_{r} \in \mathbb{Z} \text {, g.c.d. }\left(n_{0}, n_{p}, n_{r}\right)=1 \text {, and } n_{0}>0 \text {. }
$$

When (2.41) holds, shock surface oscillations with phase $\phi_{0}$ interact with interior $\phi_{q}$ oscillations to produce new $\phi_{p}$ oscillations. Again, there are at most finitely many normalized relations of this type.

\subsection{The large system for individual modes.}

To carry out a mode-by-mode analysis of the profile equations (2.16), we recall the decomposition (2.15) of the projector $\mathbb{E}^{\prime}$

$$
\mathbb{E}^{\prime}=\mathbb{E}_{0}^{\prime}+\sum_{m=1}^{M} \mathbb{E}_{m}^{\prime} .
$$


For each $m \in\{1, \ldots, M\}$ we let

$$
\ell_{m, k}, k=1, \ldots, \nu_{k_{m}}
$$

denote a basis of real vectors for the left eigenspace of

$$
i \mathcal{A}(\beta)=A_{d}^{-1}(0)\left(\underline{\tau} I+\sum_{j=0}^{d-1} A_{j}(0) \underline{\eta}_{j}\right)
$$

associated to the eigenvalue $-\underline{\omega}_{m}$, chosen to satisfy

$$
\ell_{m, k} \cdot r_{m^{\prime}, k^{\prime}}=\left\{\begin{array}{l}
1, \text { if } m=m^{\prime} \text { and } k=k^{\prime} \\
0, \text { otherwise }
\end{array} .\right.
$$

For $v \in \mathbb{C}^{N}$ set

$$
P_{m, k} v=\left(\ell_{m, k} \cdot v\right) r_{m, k} \text { (no complex conjugation here) . }
$$

We can now write

$$
\mathbb{E}^{\prime}=\mathbb{E}_{0}^{\prime}+\sum_{m=1}^{M} \sum_{k=1}^{\nu_{k_{m}}} \mathbb{E}_{m, k}^{\prime}
$$

where

$$
\mathbb{E}_{m, k}^{\prime}\left(V_{\alpha} e^{i \alpha \cdot \theta}\right):=\left\{\begin{array}{l}
\left(P_{m, k} V_{\alpha}\right) e^{i n_{\alpha} \cdot \theta_{m}}, \alpha \in \mathcal{C}_{m}^{\prime} \backslash 0 \\
0, \text { otherwise }
\end{array} ; \text { that is, } \mathbb{E}_{m, k}^{\prime}=P_{m, k} \mathbb{E}_{m}^{\prime} .\right.
$$

We will obtain a system of equations for the profiles $\underline{v}(x), \sigma_{m, k}\left(x, \theta_{m}\right)$ appearing in (2.19) by applying the projectors $\mathbb{E}_{0}^{\prime}, \mathbb{E}_{m, k}^{\prime}$ to $(2.16)(\mathrm{b})$.

The following lemma, which is a variation on a well-known result [10], is included for the sake of completeness:

Lemma 2.11. Suppose $\mathbb{E}^{\prime} \mathcal{V}^{0}=\mathcal{V}^{0}$. Then

$$
\mathbb{E}_{m, k}^{\prime}\left(\tilde{L}\left(\partial_{x}\right) \mathcal{V}^{0}\right)=\left(X_{\phi_{m}} \sigma_{m, k}\right) r_{m, k}
$$

where $X_{\phi_{m}}$ is the characteristic vector field associated to $\phi_{m}$ :

$$
X_{\phi_{m}}:=\partial_{x_{d}}+\sum_{j=0}^{d-1}-\partial_{\xi_{j}} \omega_{m}(\beta) \partial_{x_{j}} .
$$

Observe that since

$$
\partial_{\xi_{0}} \omega_{m}(\beta)=-\frac{1}{\partial_{\xi_{d}} \lambda_{k_{m}}\left(\underline{\eta}, \underline{\omega}_{m}\right)}, \partial_{\xi_{j}} \omega_{m}(\beta)=-\frac{\partial_{\xi_{j}} \lambda_{k_{m}}\left(\underline{\eta}, \underline{\omega}_{m}\right)}{\partial_{\xi_{d}} \lambda_{k_{m}}\left(\underline{\eta}_{m}\right)}, j=1, \ldots, d-1,
$$

$X_{\phi_{m}}$ is a constant multiple of the vector field computed in Lax [10]. 
Proof. For $\xi^{\prime} \in \mathcal{H}$ near $\beta$, let $-\omega_{m}\left(\xi^{\prime}\right)$ be the eigenvalues $i \mathcal{A}\left(\xi^{\prime}\right)(2.44)$ and $P_{m}\left(\xi^{\prime}\right)$ the corresponding projectors. Differentiate the equation

$$
\left(\omega_{m}\left(\xi^{\prime}\right) I+\sum_{j=0}^{d-1} \tilde{A}_{j}(0) \xi_{j}\right) P_{m}\left(\xi^{\prime}\right)=0
$$

with respect to $\xi_{j}$, evaluate at $\beta$, apply $P_{m}$ on the left to obtain

$$
P_{m} \tilde{A}_{j}(0) P_{m}=-\partial_{\xi_{j}} \omega_{m}(\beta) P_{m} .
$$

Since $\ell_{m^{\prime}, k^{\prime}} P_{m}=\delta_{m, m^{\prime}} \ell_{m^{\prime}, k^{\prime}}$ and $P_{m} r_{m, k}=r_{m, k}$, with (2.45) this implies

$$
\ell_{m^{\prime}, k^{\prime}} \cdot \tilde{A}_{j}(0) r_{m, k}=\left\{\begin{array}{l}
-\partial_{\xi_{j}} \omega_{m}(\underline{\tau}, \underline{\eta}), \text { if } m=m^{\prime}, k=k^{\prime} \\
0, \text { otherwise }
\end{array}\right.
$$

The map in the following definition will allow us to write down simple integrals that pick out the resonances associated to relations like (2.40).

Definition 2.12. Consider an ordered triple $\left(\phi_{q}, \phi_{p}, \phi_{r}\right)$ of resonant phases satisfying the normalized relation (2.40):

$$
n_{q} \phi_{q}=n_{p} \phi_{p}+n_{r} \phi_{r}
$$

For any $f \in H_{T}^{s}\left(x, \theta_{q}\right)$ define $f_{n_{q}} \in H_{T}^{s}\left(x, \theta_{q}\right)$ to be the image of $f$ under the preparation map

$$
f\left(x, \theta_{q}\right)=\sum_{k \in \mathbb{Z}} f_{k}(x) e^{i k \theta_{q}} \rightarrow \sum_{k \in \mathbb{Z}} f_{k n_{q}}(x) e^{i k n_{q} \theta_{q}} .
$$

Suppose $s>\frac{d+3}{2}+1$ and that $\sigma_{q, k}, \sigma_{r, k^{\prime}}$ are both in $H_{T}^{s}\left(\overline{\mathbb{R}}_{+}^{d+1} \times \mathbb{T}\right)$. Consider the six rearrangements of the equation (2.55):

$$
\begin{aligned}
& n_{q} \phi_{q}=n_{p} \phi_{p}+n_{r} \phi_{r}, \quad n_{q} \phi_{q}=n_{r} \phi_{r}+n_{p} \phi_{p}, \quad n_{p} \phi_{p}=n_{q} \phi_{q}-n_{r} \phi_{r}, \\
& \quad n_{p} \phi_{p}=-n_{r} \phi_{r}+n_{q} \phi_{q}, \quad n_{r} \phi_{r}=n_{q} \phi_{q}-n_{p} \phi_{p}, \quad n_{r} \phi_{r}=-n_{p} \phi_{p}+n_{q} \phi_{q} .
\end{aligned}
$$

To these equations we associate, in the same order, the six families of prepared integrals:

$$
\begin{aligned}
& I_{n_{q}, n_{p}, n_{r}}^{k, k^{\prime}}\left(x, \theta_{p}\right):= \\
& \quad \frac{1}{2 \pi} \int_{0}^{2 \pi}\left(\sigma_{q, k}\right)_{n_{q}}\left(x, \frac{n_{p}}{n_{q}} \theta_{p}+\frac{n_{r}}{n_{q}} \theta_{r}\right) \partial_{\theta_{r}} \sigma_{r, k^{\prime}}\left(x, \theta_{r}\right) d \theta_{r}, \quad k \in\left\{1, \ldots, \nu_{k_{q}}\right\}, k^{\prime} \in\left\{1, \ldots, \nu_{k_{r}}\right\}, \\
& \ldots, \\
& I_{n_{r},-n_{p}, n_{q}}^{k, \prime^{\prime}}\left(x, \theta_{p}\right):= \\
& \quad \frac{1}{2 \pi} \int_{0}^{2 \pi}\left(\sigma_{r, k}\right)_{n_{r}}\left(x, \frac{-n_{p}}{n_{r}} \theta_{p}+\frac{n_{q}}{n_{r}} \theta_{q}\right) \partial_{\theta_{q}} \sigma_{q, k^{\prime}}\left(x, \theta_{q}\right) d \theta_{q}, \quad k \in\left\{1, \ldots, \nu_{k_{r}}\right\}, k^{\prime} \in\left\{1, \ldots, \nu_{k_{q}}\right\} .
\end{aligned}
$$


The next Proposition makes precise the sense in which the prepared integrals (2.58) pick out resonances associated to the ordered triple $\left(\phi_{q}, \phi_{p}, \phi_{r}\right)$.

Proposition 2.13. Suppose $s>\frac{d+3}{2}+1$ and that $\sigma_{q, k}, \sigma_{r, k^{\prime}} \in H_{T}^{s}\left(\overline{\mathbb{R}}_{+}^{d+1} \times \mathbb{T}\right)$ have Fourier series

$$
\sigma_{q, k}\left(x, \theta_{q}\right)=\sum_{j \in \mathbb{Z}} a_{j}(x) e^{i j \theta_{q}} \text { and } \sigma_{r, k^{\prime}}\left(x, \theta_{r}\right)=\sum_{j \in \mathbb{Z}} b_{j}(x) e^{i j \theta_{r}}
$$

The prepared integral $I_{n_{q}, n_{p}, n_{r}}^{k, k^{\prime}}\left(x, \theta_{p}\right)(2.58)$ belongs to $H_{T}^{s-1}\left(x, \theta_{p}\right)$ and has Fourier series

$$
I_{n_{q}, n_{p}, n_{r}}^{k, k^{\prime}}\left(x, \theta_{p}\right)=\sum_{j \in \mathbb{Z}} a_{j n_{q}}(x) b_{-j n_{r}}(x) i \cdot\left(-j n_{r}\right) e^{i j n_{p} \theta_{p}} \quad(i=\sqrt{-1}) .
$$

Similar formulas hold for the other integrals in (2.58).

Proof. Observe that $\left(\sigma_{q, k}\right)_{n_{q}}$ and $\partial_{\theta_{r}} \sigma_{r, k^{\prime}}$ both belong to $H_{T}^{s-1}\left(\overline{\mathbb{R}}_{+}^{d+1} \times \mathbb{T}^{2}\right)$; their product then lies in the same space since $s-1>\frac{d+3}{2}$, and thus the partial sums of

$$
J\left(x, \theta_{p}, \theta_{r}\right):=\left(\sigma_{q, k}\right)_{n_{q}}\left(x, \frac{n_{p}}{n_{q}} \theta_{p}+\frac{n_{r}}{n_{q}} \theta_{r}\right) \partial_{\theta_{r}} \sigma_{r, k^{\prime}}\left(x, \theta_{r}\right)
$$

converge in $H_{T}^{s-1}\left(\overline{\mathbb{R}}_{+}^{d+1} \times \mathbb{T}^{2}\right)$. An application of the Cauchy-Schwarz inequality shows that the partial sums of

$$
I_{n_{q}, n_{p}, n_{r}}^{k, k^{\prime}}\left(x, \theta_{p}\right)=\frac{1}{2 \pi} \int_{0}^{2 \pi} J\left(x, \theta_{p}, \theta_{r}\right) d \theta_{r}
$$

converge in $H_{T}^{s-1}\left(\overline{\mathbb{R}}_{+}^{d+1} \times \mathbb{T}\right)$. The formula (2.60) follows by term-by-term integration of the partial sums of $J$.

We can now write out the large system for the profiles $\underline{v}(x), \sigma_{m, k}\left(x, \theta_{m}\right)$ appearing in the expansion of $\mathcal{V}^{0}$.

Interior equations. We assume for the moment that there exists only one ordered triple of resonant phases, say $\left(\phi_{q}, \phi_{p}, \phi_{r}\right)$. The following interior equations are obtained by applying the projectors $\mathbb{E}_{0}^{\prime}, \mathbb{E}_{p, l}^{\prime}$ to $(2.16)(\mathrm{b})$ :

$$
\tilde{L}\left(\partial_{x}\right) \underline{v}+\sum_{j=0}^{d-1} \sum_{m=1}^{M} \sum_{k, k^{\prime}=1}^{\nu_{k_{m}}} \frac{1}{2 \pi}\left(\int_{0}^{2 \pi} \sigma_{m, k}\left(x, \theta_{m}\right) \partial_{\theta_{m}} \sigma_{m, k^{\prime}}\left(x, \theta_{m}\right) d \theta_{m}\right) R_{j, m}^{k, k^{\prime}}=F(0) \underline{v},
$$


(a) $X_{\phi_{p}} \sigma_{p, l}\left(x, \theta_{p}\right)+\sum_{j=0}^{d-1} \sum_{k^{\prime}=1}^{\nu_{k_{p}}} a_{p, l, j}^{k^{\prime}}(\underline{v}) \partial_{\theta_{p}} \sigma_{p, k^{\prime}}\left(x, \theta_{p}\right)+$

(b) $\sum_{j=0}^{d-1} \sum_{k=1}^{\nu_{k_{p}}} \sum_{k^{\prime}=1}^{\nu_{k_{p}}} b_{p, l, j}^{k, k^{\prime}} \sigma_{p, k}\left(x, \theta_{p}\right) \partial_{\theta_{p}} \sigma_{p, k^{\prime}}\left(x, \theta_{p}\right)-\sum_{j=0}^{d-1} \sum_{k=1}^{\nu_{k_{p}}} \sum_{k^{\prime}=1}^{\nu_{k_{p}}} b_{p, l, j}^{k, k^{\prime}} \frac{1}{2 \pi} \int_{0}^{2 \pi} \sigma_{p, k}\left(x, \theta_{p}\right) \partial_{\theta_{p}} \sigma_{p, k^{\prime}}\left(x, \theta_{p}\right) d \theta_{p}+$

(c) $\quad \sum_{j=0}^{d-1} \sum_{k=1}^{\nu_{k_{q}}} \sum_{k^{\prime}=1}^{\nu_{k_{r}}} c_{p, l, j}^{k, k^{\prime}} I_{n_{q}, n_{p}, n_{r}}^{k, k^{\prime}}\left(x, \theta_{p}\right)+\sum_{j=0}^{d-1} \sum_{k=1}^{\nu_{k_{r}}} \sum_{k^{\prime}=1}^{\nu_{k_{q}}} d_{p, l, j}^{k, k^{\prime}} I_{n_{r},-n_{p}, n_{q}}^{k, k^{\prime}}\left(x, \theta_{p}\right)$

$(d)$

$$
=\sum_{k=1}^{\nu_{k_{p}}} e_{p, l}^{k} \sigma_{p, k}\left(x, \theta_{p}\right)
$$

together with additional equations beginning with $X_{\phi_{m}} \sigma_{m, k}$ for the other $(m, k)$. The equations (2.63), (2.64) are explained further in the following Remark.

Remark 2.14. 1 . The constant vectors $R_{j, m}^{k, k^{\prime}}$ appearing in (2.63) are given by

$$
R_{j, m}^{k, k^{\prime}}=\beta_{j}\left(\partial_{u} \tilde{A}_{j}(0) \cdot r_{m, k}\right) r_{m, k^{\prime}}
$$

The constant scalars $b_{p, l, j}^{k, k^{\prime}}, c_{p, l, j}^{k, k^{\prime}}, d_{p, l, j}^{k, k^{\prime}}, e_{p, l}^{k}$, and the coefficients of the scalar linear function of $\underline{v}, a_{p, l, j}^{k}(\underline{v})$, in (2.64) are given by similar formulas, but now involving dot products with the vector $\ell_{p, l}$.

2. The characteristic vector fields $X_{\phi_{p}}$ were defined in (2.50). The second term in (2.64)(a) describes the interaction between $\underline{v}$ and $\left(\mathcal{V}^{0}\right)^{*}=\mathcal{V}^{0}-\underline{v}$.

3. The sum being subtracted in (2.64)(b) is the mean of the first sum. Each of lines (a),(b),(c),(d) in (2.64) has mean zero.

4. The prepared integrals appearing in $(2.64)(\mathrm{c})$ are the integrals associated to the first and last equations in (2.57).

5. The equations in the system beginning with the terms $X_{\phi_{q}} \sigma_{q, l^{\prime}}, X_{\phi_{r}} \sigma_{r, l^{\prime \prime}}$ are similar to (2.64). In particular, each equation involves two sums of prepared integrals similar to those in $(2.64)(\mathrm{c})$. The equations beginning with terms $X_{\phi_{m}} \sigma_{m, k}, m \in\{1, \ldots, M\} \backslash\{q, p, r\}$ involve no prepared integrals.

6 . If there is more than one ordered triple $\left(\phi_{q}, \phi_{p}, \phi_{r}\right)$ of resonant phases, more prepared integrals will appear in the system. For example, if there is exactly one more ordered triple, say $\left(\phi_{p}, \phi_{s}, \phi_{t}\right)$, containing $\phi_{p}$, two additional sums of prepared integrals will appear in $(2.64)(\mathrm{c})$. 
Boundary equations. From the boundary equation (2.16)(c) we obtain

(a) $B(0) \underline{v}=\underline{G}\left(x^{\prime}\right)$

(b) $B(0) \mathcal{V}^{0 *}\left(x^{\prime}, 0, \theta_{0}, \ldots, \theta_{0}\right)=$

$$
B(0)\left(\sum_{m \in \mathcal{I}} \sum_{k=1}^{\nu_{k_{m}}} \sigma_{m, k}\left(x^{\prime}, 0, \theta_{0}\right) r_{m, k}+\sum_{m \in \mathcal{O}} \sum_{k=1}^{\nu_{k_{m}}} \sigma_{m, k}\left(x^{\prime}, 0, \theta_{0}\right) r_{m, k}\right)=G^{*}\left(x^{\prime}, \theta_{0}\right) \text {. }
$$

Lemma 1.10 implies that the set $\left\{r_{m, k}: k \in\left\{1, \ldots, \nu_{k_{m}}\right\}, m \in \mathcal{I}\right\}$ is a basis for $\mathbb{E}^{s}(\underline{\tau}, \underline{\eta})$. By uniform stability, Assumption 1.6, we can therefore rewrite (2.66)(b) as

$$
\begin{aligned}
& \left(\sigma_{m, k}\left(x^{\prime}, 0, \theta_{0}\right) ; m \in \mathcal{I}, k \in\left\{1, \ldots, \nu_{k_{m}}\right\}\right)= \\
& \mathcal{B}\left(G^{*}\left(x^{\prime}, \theta_{0}\right), \sigma_{m, k}\left(x^{\prime}, 0, \theta_{0}\right) ; m \in \mathcal{O}, k \in\left\{1, \ldots, \nu_{k_{m}}\right\}\right),
\end{aligned}
$$

where $\mathcal{B}$ is a well-determined linear function of its arguments.

Remark 2.15. We point out here one of the differences between our profile equations (2.64) and the profile equations derived in earlier works on geometric optics such as $[14,21]$ for fixed and free boundary problems for systems of conservation laws. Profile equations were derived in [14] under the assumption that the matrices $A_{j}$ appearing in the system (1.2) satisfied

$$
A_{j}=d f_{j},
$$

where the $f_{j}: \mathbb{R}^{N} \rightarrow \mathbb{R}^{N}$ were fluxes in a system of conservation laws, by substituting the approximate solution ansatz into (1.3). In that case for each $j$ the $N$ matrix components of $\partial_{u} A_{j}$ are given by the Hessian matrices of the $N$ components of $f_{j}$. The symmetry of those matrix components implied that the analogue of our equation (2.63) did not involve any of the $\sigma_{m, k}$. Thus, the boundary problem satisfied by $\underline{v}$ decoupled from the other equations and $\underline{v}$ could be determined first, independently of the $\sigma_{m, k}$. In this paper we do not assume that the $A_{j}$ satisfy (2.68), so we do not obtain the same decoupling. In the strictly hyperbolic case the equation (2.63) shows that decoupling occurs even without the condition (2.68), since $k=k^{\prime}=1$ then and the integrals in (2.63) are zero.

The large system. The interior equations (2.63),(2.64) together with the boundary equations (2.66) and the initial conditions,

$$
\underline{v}=0 \text { and } \sigma_{m, k}=0 \text { in } t \leq 0 \text { for all } m, k,
$$

defined a forward, quasilinear, integro-differential, initial boundary-value problem for the unknowns

$$
\underline{v},\left(\sigma_{m, k}\right)_{m \in\{1, \ldots, M\}, k \in\left\{1, \ldots, \nu_{k_{m}}\right\}} .
$$

This large system is equivalent to the problem (2.16). The solution can be obtained by an iterative argument based on an a priori estimate for a suitable linearization described in the next section. 


\subsection{Solution of the large system.}

The iteration scheme and the linearized problem for solving the large system can be presented simultaneously as follows. Letting $\left(\underline{v}^{n}, \sigma_{m, k}^{n}\right)$ denote the $n$-th iterate and taking $\left(\underline{v}^{0}, \sigma_{m, k}^{0}\right)=0$, we define the $(n+1)$-st iterate as the solution to the coupled, linear, initial boundary-value problems

(a) $\tilde{L}\left(\partial_{x}\right) \underline{v}^{n+1}=\sum_{j=0}^{d-1} \sum_{m=1}^{M} \sum_{k, k^{\prime}=1}^{\nu_{k_{m}}} \frac{1}{2 \pi}\left(\int_{0}^{2 \pi} \sigma_{m, k}^{n}\left(x, \theta_{m}\right) \partial_{\theta_{m}} \sigma_{m, k^{\prime}}^{n+1}\left(x, \theta_{m}\right) d \theta_{m}\right) R_{j, m}^{k, k^{\prime}}+F(0) \underline{v}^{n}$

(b) $B(0) \underline{v}^{n+1}=\underline{G}$

(c) $\underline{v}^{n+1}=0$ in $t \leq 0$

and

$$
\begin{aligned}
& \text { (a) } X_{\phi_{p}} \sigma_{p, l}^{n+1}\left(x, \theta_{p}\right)+\sum_{j=0}^{d-1} \sum_{k^{\prime}=1}^{\nu_{k_{p}}} a_{p, l, j}^{k^{\prime}}\left(\underline{v}^{n}\right) \partial_{\theta_{p}} \sigma_{p, k^{\prime}}^{n+1}\left(x, \theta_{p}\right)+ \\
& \sum_{j=0}^{d-1} \sum_{k=1}^{\nu_{k_{p}}} \sum_{k^{\prime}=1}^{\nu_{k_{p}}} b_{p, l, j}^{k, k^{\prime}} \sigma_{p, k}^{n}\left(x, \theta_{p}\right) \partial_{\theta_{p}} \sigma_{p, k^{\prime}}^{n+1}\left(x, \theta_{p}\right)-\sum_{j=0}^{d-1} \sum_{k=1}^{\nu_{k_{p}}} \sum_{k^{\prime}=1}^{\nu_{k_{p}}} b_{p, l, j}^{k, k^{\prime}} \frac{1}{2 \pi} \int_{0}^{2 \pi} \sigma_{p, k}^{n}\left(x, \theta_{p}\right) \partial_{\theta_{p}} \sigma_{p, k^{\prime}}^{n+1}\left(x, \theta_{p}\right) d \theta_{p}+ \\
& \quad \sum_{j=0}^{d-1} \sum_{k=1}^{\nu_{k_{q}}} \sum_{k^{\prime}=1}^{\nu_{k_{r}}} c_{p, l, j}^{k, k^{\prime}} \frac{1}{2 \pi} \int_{0}^{2 \pi}\left(\sigma_{q, k}^{n}\right)_{n_{q}}\left(x, \frac{n_{p}}{n_{q}} \theta_{p}+\frac{n_{r}}{n_{q}} \theta_{r}\right) \partial_{\theta_{r}} \sigma_{r, k^{\prime}}^{n+1}\left(x, \theta_{r}\right) d \theta_{r}+
\end{aligned}
$$$$
\sum_{j=0}^{d-1} \sum_{k=1}^{\nu_{k_{r}}} \sum_{k^{\prime}=1}^{\nu_{k_{q}}} d_{p, l, j}^{k, k^{\prime}} \frac{1}{2 \pi} \int_{0}^{2 \pi}\left(\sigma_{r, k}^{n}\right)_{n_{r}}\left(x, \frac{-n_{p}}{n_{r}} \theta_{p}+\frac{n_{q}}{n_{r}} \theta_{q}\right) \partial_{\theta_{q}} \sigma_{q, k^{\prime}}^{n+1}\left(x, \theta_{q}\right) d \theta_{q}=\sum_{k=1}^{\nu_{k_{p}}} e_{p, l}^{k} \sigma_{p, k}^{n}\left(x, \theta_{p}\right) \text {, }
$$$$
\text { (b) } B(0)\left(\sum_{m \in \mathcal{I}} \sum_{k=1}^{\nu_{k_{m}}} \sigma_{m, k}^{n+1}\left(x^{\prime}, 0, \theta_{0}\right) r_{m, k}+\sum_{m \in \mathcal{O}} \sum_{k=1}^{\nu_{k_{m}}} \sigma_{m, k}^{n+1}\left(x^{\prime}, 0, \theta_{0}\right) r_{m, k}\right)=G^{*}\left(x^{\prime}, \theta_{0}\right)
$$

(c) $\sigma_{m, k}^{n+1}=0$ in $t \leq 0$ for all $m, k$,

where the remaining interior equations in (2.72)(a) are added following the prescriptions of Remark 2.14, parts 5 and 6.

Remark 2.16. As we will see below in the proof of Proposition 2.19, the terms involving integrals in the interior equations of (2.71) and (2.72) present no obstacle to obtaining $L^{2}$ estimates for the coupled systems. However, the sum in the first line of (2.72)(a) and the first sum in the next line would present a serious obstacle if it were not possible to rewrite them in a symmetric form. The next Proposition shows there is a symmetry in the coefficients that appears after regrouping. In preparation we note that

$$
b_{p, l, j}^{k, k^{\prime}}=\ell_{p, l} \cdot \beta_{j}\left(\partial_{u} \tilde{A}_{j}(0) r_{p, k}\right) r_{p, k^{\prime}} .
$$


Expanding $\underline{v}^{n}=\sum_{p, k} \underline{v}_{p, k}^{n} r_{p, k}$ we can rewrite

$$
a_{p, l, j}^{k^{\prime}}\left(\underline{v}^{n}\right)=\sum_{k} b_{p, l, j}^{k, k^{\prime}} \underline{v}_{p, k}^{n}
$$

with the same coefficients $b_{p, l, j}^{k, k^{\prime}}$.

Definition 2.17. For u near 0 let $-\underline{\omega}_{m}(u)$ be the eigenvalues of

$$
i \mathcal{A}(u, \beta):=A_{d}^{-1}(u)\left(\underline{\tau} I+\sum_{j=0}^{d-1} A_{j}(u) \underline{\eta}_{j}\right),
$$

and $P_{m}(u)$ the corresponding projectors.

The functions $\underline{\omega}_{m}(u)$ and $P_{m}(u)$ are $C^{\infty}$ for $u$ near 0 since $\beta$ then belongs to the hyperbolic region of $\mathcal{A}\left(u, \xi^{\prime}\right)$.

Proposition 2.18. Let $w \in \mathbb{R}^{N}$ be expanded as $w=\sum_{p, k} w_{p, k} r_{p, k}=\sum_{p} w_{p}$ and define

$$
B_{l, k^{\prime}}^{p}(w)=\sum_{j=0}^{d-1} \sum_{k=1}^{\nu_{k_{p}}} b_{p, l, j}^{k, k^{\prime}} w_{p, k}=\ell_{p, l} \sum_{j=0}^{d-1} \beta_{j}\left(\partial_{u} \tilde{A}_{j}(0) \cdot w_{p}\right) r_{p, k^{\prime}}
$$

Then

$$
B_{l, k^{\prime}}^{p}(w)=\left\{\begin{array}{l}
-\partial_{u} \underline{\omega}_{p}(0) \cdot w_{p}, k^{\prime}=l \\
0, \text { otherwise }
\end{array} .\right.
$$

Proof. The proof is quite similar to that of Lemma 2.11. We differentiate the equation

$$
\left(\omega_{p}(u) I+\sum_{j=0}^{d-1} \beta_{j} \tilde{A}_{j}(u)\right) P_{p}(u)=0
$$

with respect to $u$ in the direction $w_{p}$, evaluate at $u=0$, and apply $P_{p}=P_{p}(0)$ on the left to obtain

$$
P_{p} \sum_{j=0}^{d-1} \beta_{j}\left(\partial_{u} \tilde{A}_{j}(0) \cdot w_{p}\right) P_{p}=\left(-\partial_{u} \underline{\omega}_{p}(0) \cdot w_{p}\right) P_{p},
$$

The second equality in (2.76) and (2.79) imply (2.77).

Spaces of profiles. Set $b \Omega_{T}:=\left\{\left(x^{\prime}, \theta_{0}\right):-\infty<t<T, \theta_{0} \in \mathbb{T}\right\}$ as before and let $\Omega_{T, \theta}:=\left\{(x, \theta):-\infty<t<T, x_{d} \geq 0, \theta \in \mathbb{T}^{M}\right\}$. For $m \in\{0,1,2, \ldots\}, T>0$ and $\gamma>0$ 
define

$$
\begin{aligned}
\mathbb{H}_{T}^{m} & =\left\{U(x, \theta):|U|_{m, T}:=\sum_{|\alpha| \leq m}\left|\partial_{x, \theta}^{\alpha} U\right|_{L^{2}\left(\Omega_{T, \theta}\right)}<\infty\right\} \\
H_{T}^{m} & =\left\{V\left(x^{\prime}, \theta_{0}\right):\langle V\rangle_{m, T}:=\sum_{|\alpha| \leq m}\left|\partial_{x^{\prime}, \theta_{0}}^{\alpha} V\right|_{L^{2}\left(b \Omega_{T, \theta_{0}}\right)}<\infty\right\} \\
\mathbb{H}_{\gamma, T}^{m} & =\left\{U(x, \theta):|U|_{m, \gamma, T}:=\sum_{|\alpha| \leq m} \gamma^{m-|\alpha|}\left|e^{-\gamma t} \partial_{x, \theta}^{\alpha} U\right|_{L^{2}\left(\Omega_{T, \theta}\right)}<\infty\right\}
\end{aligned}
$$

and $H_{\gamma, T}^{m}$ is defined similarly on $b \Omega_{T}$. We set $|U(x, \theta)|_{\infty}:=|U(x, \theta)|_{L^{\infty}\left(\Omega_{T, \theta}\right)}$ and define

$$
\mathbb{W}_{T}^{1, \infty}=\left\{U(x, \theta):|U|_{1, \infty}:=\sum_{|\alpha| \leq 1}\left|\partial_{x, \theta}^{\alpha} U\right|_{L^{\infty}\left(\Omega_{T, \theta}\right)}<\infty\right\} .
$$

Estimates for the coupled systems. We can now state the main existence result for solutions

$$
\mathcal{V}^{0, n+1}(x, \theta)=\underline{v}^{n+1}(x)+\sum_{m=1}^{M} \sum_{k=1}^{\nu_{k_{m}}} \sigma_{m, k}^{n+1}\left(x, \theta_{m}\right) r_{m, k}
$$

of the coupled linear systems (2.71)-(2.72).

Proposition 2.19. Let $T>0, m>\frac{d+3}{2}+1$ and suppose that $G\left(x^{\prime}, \theta_{0}\right) \in H_{T}^{m}$ and $\mathcal{V}^{0, n} \in \mathbb{H}_{T}^{m}$ both vanish in $t \leq 0$. The coupled system (2.71)-(2.72) has a unique solution $\mathcal{V}^{0, n+1} \in \mathbb{H}_{T}^{m}$ vanishing in $t \leq 0$. Moreover, there exist increasing functions, $\gamma_{0}(K)$ and $C(K)$, of $K=$ $\left|\mathcal{V}^{0, n}\right|_{m, T}$ such that for $\gamma>\gamma_{0}(K)$ we have

$$
\left|\mathcal{V}^{0, n+1}\right|_{m, \gamma, T}+\frac{\left\langle\mathcal{V}^{0, n+1}\right\rangle_{m, \gamma, T}}{\sqrt{\gamma}} \leq C(K)\left(\frac{\left|\mathcal{V}^{0, n}\right|_{m, \gamma, T}}{\gamma}+\frac{\langle G\rangle_{m, \gamma, T}}{\sqrt{\gamma}}\right) .
$$

Proof. 1. $L^{2}$ estimate. Anticipating the extra forcing terms that arise in the higher derivative estimates, we first prove an $L^{2}$ a priori estimate for the coupled systems in the case where forcing terms $\underline{F}(x)$ and $f_{p, l}\left(x, \theta_{p}\right)$ are added to the right sides of (2.71)(a) and $(2.72)$ (a) respectively. Setting $F(x, \theta):=\underline{F}(x)+\sum_{p, k} f_{p, k}\left(x, \theta_{p}\right) r_{p, k}$ we have

$$
\left|\mathcal{V}^{0, n+1}\right|_{0, \gamma, T}+\frac{\left\langle\mathcal{V}^{0, n+1}\right\rangle_{0, \gamma, T}}{\sqrt{\gamma}} \leq C\left(K^{\prime}\right)\left(\frac{|F|_{0, \gamma, T}}{\gamma}+\frac{\left|\mathcal{V}^{0, n}\right|_{0, \gamma, T}}{\gamma}+\frac{\langle G\rangle_{0, \gamma, T}}{\sqrt{\gamma}}\right),
$$

where

$$
K^{\prime}:=\max \left(\left|\underline{v}^{n}\right|_{1, \infty},\left|\sigma_{p, k}^{n}\right|_{1, \infty},\left|\left(\sigma_{r, k}^{n}\right)_{n_{r}}\right|_{1, \infty}\right),
$$

the max being taken over all $(p, k)$ and over all prepared profiles appearing in the system (2.72)(a). Observe that $K^{\prime}$ is finite since, for example,

$$
\left|\left(\sigma_{r, k}^{n}\right)_{n_{r}}\right|_{1, \infty} \leq C\left|\left(\sigma_{r, k}^{n}\right)_{n_{r}}\right|_{m, T} \leq C\left|\left(\sigma_{r, k}^{n}\right)\right|_{m, T} \leq C\left|\mathcal{V}^{0, n}\right|_{m, T} .
$$


With $\mathcal{V}^{0, n+1}=\underline{v}^{n+1}+\left(\mathcal{V}^{0, *}\right)^{n+1}$ the estimate (2.84) follows immediately by adding the intermediate estimates

$$
\begin{aligned}
& \text { (a) }\left|\underline{v}^{n+1}\right|_{0, \gamma, T}+\frac{\left\langle\underline{v}^{n+1}\right\rangle_{0, \gamma, T}}{\sqrt{\gamma}} \leq C\left(K^{\prime}\right)\left(\frac{\left|\left(\mathcal{V}^{0, *}\right)^{n+1}\right|_{0, \gamma, T}}{\gamma}+\frac{|\underline{F}|_{0, \gamma, T}}{\gamma}+\frac{\left|\underline{v}^{n}\right|_{0, \gamma, T}}{\gamma}+\frac{\langle\underline{G}\rangle_{0, \gamma, T}}{\sqrt{\gamma}}\right) . \\
& \text { (b) }\left|\left(\mathcal{V}^{0, *}\right)^{n+1}\right|_{0, \gamma, T}+\frac{\left\langle\left(\mathcal{V}^{0, *}\right)^{n+1}\right\rangle_{0, \gamma, T}}{\sqrt{\gamma}} \leq C\left(K^{\prime}\right)\left(\frac{\left|F^{*}\right|_{0, \gamma, T}}{\gamma}+\frac{\left|\left(\mathcal{V}^{0, *}\right)^{n}\right|_{0, \gamma, T}}{\gamma}+\frac{\left\langle G^{*}\right\rangle_{0, \gamma, T}}{\sqrt{\gamma}}\right)
\end{aligned}
$$

and absorbing terms from the right.

After integrating by parts in the sum on the right side of (2.71)(a), one obtains (2.87)(a) directly from the standard Kreiss estimate $[9,3]$ for the uniformly stable problem $\left(L\left(\partial_{x}\right), B(0)\right)$. To obtain (2.87)(b) we first integrate by parts in all the terms of (2.72)(a) involving integrals to switch the $\partial_{\theta_{p}}$ (or $\partial_{\theta_{q}}$ or $\partial_{\theta_{r}}$ ) onto the $n$-th iterate. Next use Proposition 2.18 to rewrite the sum of the second and third terms in (2.72)(a) as

$$
\sum_{l=1}^{\nu_{k_{p}}} B_{l, l}^{p}\left(\underline{v}^{n}+\left(\mathcal{V}^{0, *}\right)^{n}\right) \partial_{\theta_{p}} \sigma_{p, l}^{n+1}\left(x, \theta_{p}\right) .
$$

Observe that $e^{-\gamma t}\left(\mathcal{V}^{0, *}\right)^{n+1}$ satisfies a system just like (2.72), except that $\partial_{t}$ in $X_{\phi_{p}}$ is replaced by $\left(\partial_{t}+\gamma\right)$, and $G^{*}$ is multiplied by $e^{-\gamma t}$. Multiply equation (2.72)(a) by $e^{-\gamma t} \sigma_{p, l}^{n+1}\left(x, \theta_{p}\right)$ and integrate $d x d \theta_{p}$. In the case when $\phi_{p}$ is incoming, using (2.88) and integration by parts we obtain straightforwardly (see Remark 2.20)

$$
\begin{gathered}
\gamma\left|\sigma_{p, l}^{n+1}\right|_{0, \gamma, T}^{2} \leq C_{p}\left\langle\sigma_{p, l}^{n+1}\right\rangle_{0, \gamma, T}^{2}+C\left(K^{\prime}\right)\left|\left(\mathcal{V}^{0, *}\right)^{n+1}\right|_{0, \gamma, T}\left|\sigma_{p, l}^{n+1}\right|_{0, \gamma, T}+ \\
\left|\left(\mathcal{V}^{0, *}\right)^{n}\right|_{0, \gamma, T}\left|\sigma_{p, l}^{n+1}\right|_{0, \gamma, T}+\left|f_{p, l}\right|_{0, \gamma, T}\left|\sigma_{p, l}^{n+1}\right|_{0, \gamma, T},
\end{gathered}
$$

where $C_{p}>0$ since $\phi_{p}$ is incoming. When $\phi_{p}$ is outgoing, $C_{p}<0$ so we obtain a similar estimate but with the boundary term $\left|C_{p}\right|\left\langle\sigma_{p, l}\right\rangle_{0, \gamma, T}^{2}$ on the left. When $\phi_{p}$ is incoming, we can use (2.67) to estimate (with obvious notation)

$$
\left\langle\sigma_{p, l}^{n+1}\right\rangle_{0, \gamma, T} \leq C\left(\left\langle\left(\mathcal{V}^{0, *}\right)_{o u t}^{n+1}\right\rangle_{0, \gamma, T}+\left\langle G^{*}\right\rangle_{0, \gamma, T}\right) .
$$

Inserting this in (2.89), summing over all modes, and absorbing terms in the usual way by taking $\gamma \geq \gamma_{0}\left(K^{\prime}\right)$ large enough, we obtain (2.84).

2. Higher derivative estimates. We return to the coupled systems (2.71)-(2.72), in their original form except for the simplification (2.88), and apply the $L^{2}$ estimate to the problem satisfied by the tangential derivatives $\gamma^{m-|\alpha|} \partial_{x^{\prime}, \theta}^{\alpha} \mathcal{V}^{0, n+1}$, where $|\alpha| \leq m$. The new source terms coming from commutators can be estimated and absorbed using the following two observations:

A. Suppose $m_{1}+m_{2}>\frac{d+3}{2}, m_{i} \geq 0$. The product $\left(a\left(x, \theta_{p}, \theta_{q}\right), b\left(x, \theta_{p}, \theta_{q}\right)\right) \rightarrow a \cdot b$ is continuous from $\mathbb{H}_{T}^{m_{1}} \times \mathbb{H}_{T}^{m_{2}} \stackrel{2}{\rightarrow} \mathbb{H}_{T}^{0}$. 
B. Suppose $\left|\alpha_{1}\right|+\left|\alpha_{2}\right| \leq|\alpha| \leq m$. Then

$$
\gamma^{m-|\alpha|}\left|e^{-\gamma t} \partial_{x, \theta}^{\alpha_{1}} u(x, \theta)\right|_{\left|\alpha_{2}\right|, T} \leq|u|_{m, \gamma, T}
$$

For example, the commutator $\gamma^{m-|\alpha|}\left|\left[B_{l, l}^{p}\left(\mathcal{V}^{0, n}\right), \partial_{x^{\prime}, \theta_{p}}^{\alpha}\right] \partial_{\theta_{p}} \sigma_{p, l}^{n+1}\right|_{0, \gamma, T}$ is dominated by a sum of terms of the form

$$
\left|\left(\partial_{x^{\prime}, \theta_{p}}^{\alpha_{1}} B_{l, l}^{p}\left(\mathcal{V}^{0, n}\right)\right)\left(\gamma^{m-|\alpha|} \partial_{x^{\prime}, \theta_{p}}^{\alpha_{2}} \partial_{\theta_{p}} \sigma_{p, l}^{n+1}\right)\right|_{0, \gamma, T},
$$

where $\left|\alpha_{1}\right|+\left|\alpha_{2}\right|=|\alpha|$ and $\left|\alpha_{1}\right| \geq 1$. By $\mathbf{A}$ (with $m_{1}=m-\left|\alpha_{1}\right|, m_{2}=\left|\alpha_{1}\right|-1$ ) and $\mathbf{B}$ we have

$$
(2.92) \leq C\left|\mathcal{V}^{0, n}\right|_{m, T}\left|\sigma_{p, l}^{n+1}\right|_{m, \gamma, T} \leq C K\left|\sigma_{p, l}^{n+1}\right|_{m, \gamma, T} .
$$

The remaining commutators are estimated similarly. Derivatives involving $\partial_{x_{d}}$ are estimated in the customary way using the tangential estimates and the fact that $x_{d}=0$ is noncharacteristic for $\tilde{L}(\partial)$ and $X_{\phi_{p}}, p=1, \ldots, M$.

3. Existence and uniqueness. Uniqueness follows from the $L^{2}$ estimate (2.84). Using the notation of $(2.16)$ and setting $\mathcal{V}^{0, n+1}=\mathcal{W}$ for now, we note that the coupled systems (2.71)-(2.72) are equivalent to the problem

$$
\begin{aligned}
& \text { a) } \mathbb{E}^{\prime} \mathcal{W}=\mathcal{W} \\
& \text { b) } \mathbb{E}^{\prime}\left(\tilde{L}\left(\partial_{x}\right) \mathcal{W}+\mathcal{M}^{\prime}\left(\mathcal{V}^{0, n}\right) \partial_{\theta} \mathcal{W}\right)=\mathbb{E}^{\prime}\left(F(0) \mathcal{V}^{0, n}\right) \text { in } x_{d} \geq 0 \\
& \text { c) } B(0) \mathcal{W}\left(x^{\prime}, 0, \theta_{0}, \ldots, \theta_{0}\right)=G\left(x^{\prime}, \theta_{0}\right) \\
& \text { d) } \mathcal{W}=0 \text { in } t<0 .
\end{aligned}
$$

The procedure by which one uses the a priori estimate (2.83) to obtain a solution of (2.95) is mostly standard, but we provide a sketch of the argument. A similar argument with more detail is given in [8], p. 83.

We keep $n$ fixed throughout this discussion and approximate $\partial_{\theta}$ by finite difference operators

$$
\partial_{\theta}^{h}=\left(\partial_{\theta_{1}}^{h}, \ldots, \partial_{\theta_{M}}^{h}\right), h>0 .
$$

Let $(2.94)_{h}$ denote the system $(2.94)$, where $\partial_{\theta}$ and $\mathcal{W}$ are replaced by $\partial_{\theta}^{h}$ and $\mathcal{W}^{h}$ respectively. For fixed $h$ we solve $(2.94)_{h}$ by the following Picard iteration in the index $\ell$ :

$$
\begin{aligned}
& \text { a) } \mathbb{E}^{\prime} \mathcal{W}_{\ell+1}^{h}=\mathcal{W}_{\ell+1}^{h} \\
& \text { b) } \mathbb{E}^{\prime}\left(\tilde{L}\left(\partial_{x}\right) \mathcal{W}_{\ell+1}^{h}\right)=\mathbb{E}^{\prime}\left(F(0) \mathcal{V}^{0, n}-\mathcal{M}^{\prime}\left(\mathcal{V}^{0, n}\right) \partial_{\theta}^{h} \mathcal{W}_{\ell}^{h}\right) \\
& \text { c) } B(0) \mathcal{W}_{\ell+1}^{h}\left(x^{\prime}, 0, \theta_{0}, \ldots, \theta_{0}\right)=G\left(x^{\prime}, \theta_{0}\right) \\
& \text { d) } \mathcal{W}_{\ell+1}^{h}=0 \text { in } t<0 .
\end{aligned}
$$


To find $\mathcal{W}_{\ell+1}^{h}$ we again use the projectors $\mathbb{E}_{0}^{\prime}, \mathbb{E}_{p, k}^{\prime}(2.47)$. The problem for $\underline{\mathcal{W}}_{\ell+1}^{h}$ is a standard Kreiss-type problem, while the problem for the components of the mean zero part $\mathcal{W}_{\ell+1}^{h, *}$ is governed by a diagonal operator with the vector fields $X_{\phi_{p}}$ (repeated as necessary) on the diagonal. Data for incoming modes at $x_{d}=0$ is prescribed using (2.67). Since the operator $\mathcal{M}^{\prime}\left(\mathcal{V}^{0, n}\right) \partial_{\theta}^{h}$ is bounded on $\mathbb{H}_{T}^{m}$, iteration yields a solution $\mathcal{W}^{h} \in \mathbb{H}_{T}^{m}$ of $(2.94)_{h}$. Next one repeats the proof of (2.83) to obtain estimates uniform in $h$ :

$$
\left|\mathcal{W}^{h}\right|_{m, \gamma, T}+\frac{\left\langle\mathcal{W}^{h}\right\rangle_{m, \gamma, T}}{\sqrt{\gamma}} \leq C(K)\left(\frac{\left|\mathcal{V}^{0, n}\right|_{m, \gamma, T}}{\gamma}+\frac{\langle G\rangle_{m, \gamma, T}}{\sqrt{\gamma}}\right)
$$

Passing to a subsequence with $h_{k} \rightarrow 0$ finally yields a solution $\mathcal{W}=\mathcal{V}^{0, n+1}$ satisfying (2.83).

Remark 2.20. In deriving (2.89) we rewrote the term $\left(\partial_{\theta_{p}} \sigma_{p, l}^{n+1}\right) \sigma_{p, l}^{n+1}$, which appeared after multiplying (2.88) by $e^{-\gamma t} \sigma_{p, l}^{n+1}$, as $\frac{1}{2} \partial_{\theta_{p}}\left(\sigma_{p, l}^{n+1}\right)^{2}$, and then integrated by parts to move the derivative onto $\mathcal{V}^{0, n}$. If Proposition 2.18 had not first provided (2.88), we could not have avoided $\theta_{p}$-derivatives of $(n+1)$-st iterates on the right side of $(2.89)$.

Next we show convergence of the iterates $\mathcal{V}^{0, n}$ to a short time solution of the nonlinear profile equations (2.16).

Proposition 2.21. Consider the profile equations (2.16), where $G \in H_{T}^{m}, m>\frac{d+3}{2}+1$, and vanishes in $t \leq 0$. For some $0<T_{0} \leq T$ the system has a unique solution $\mathcal{V}^{0} \in \mathbb{H}_{T_{0}}^{m}$.

Proof. 1. The iteration scheme (2.71)-(2.72) defines a sequence $\left(\mathcal{V}^{0, n}\right)$ in $\mathbb{H}_{T}^{m}$. Fixing $K>0$ we claim that for $T^{*}>0$ small enough,

$$
\left|\mathcal{V}^{0, n}\right|_{m, T^{*}}+\left\langle\mathcal{V}^{0, n}\right\rangle_{m, T^{*}}<K \text { for all } n \text {. }
$$

First observe that

$$
|u|_{m, \gamma, T} \leq C_{1}|u|_{m, T} \leq C_{2} e^{\gamma T}|u|_{m, \gamma, T}
$$

and fix $\gamma>\max \left(\gamma_{0}(K), 2 C(K) C_{1}\right)$ for $\gamma_{0}(\cdot)$ and $C(\cdot)$ as in Proposition 2.19. Assuming (2.98) holds for $n \leq n_{0}$, we find that it holds for $n_{0}+1$ after shrinking $T^{*}$ if necessary, using the estimate (2.83) and the fact that $G$ vanishes in $t \leq 0$. This new choice of $T^{*}$ works for all $n$.

2. Convergence of the iterates in $\mathbb{H}_{T_{0}}^{0}$ to some $\mathcal{V}^{0}$ for a possibly smaller $T_{0}>0$ now follows from (2.98) by applying $(2.83)$ when $m=0$ to the problem satisfied by $\left(\mathcal{V}^{0, n+1}-\mathcal{V}^{0, n}\right)$. In view of (2.98) and a classical argument involving weak convergence and interpolation, we thereby obtain a solution $\mathcal{V}^{0} \in \mathbb{H}_{T_{0}}^{m}$ with, in fact, a trace that lies in $H_{T_{0}}^{m}$. This argument shows that the iterates $\mathcal{V}^{0, n}$ converge to $\mathcal{V}^{0}$ in $\mathbb{H}_{T_{0}}^{m-1}$. 
Having constructed $\mathcal{V}^{0}(x, \theta) \in \mathbb{H}_{T_{0}}^{m}$ for $m$ sufficiently large (as specified below) and defining $\mathcal{U}^{0}\left(x, \theta_{0}, \xi_{d}\right) \in \mathcal{P}^{m-1}$ as in $(2.20)$, we define the approximate solution $u_{\epsilon}^{a}$ appearing in Theorem 1.15 by

$$
u_{\epsilon}^{a}(x)=\mathcal{U}^{0}\left(x, \frac{\phi_{0}}{\epsilon}, \frac{x_{d}}{\epsilon}\right)
$$

\subsection{Error analysis}

Now we are ready to prove Theorem 1.15, which shows that the approximate solution $u_{\epsilon}^{a}(x)$ converges in $L^{\infty}$ to the exact solution $u_{\epsilon}$ of Theorem 1.13 as $\epsilon \rightarrow 0$. In this section we prove the following more precise Theorem, which implies Theorem 1.15 as an immediate corollary.

Theorem 2.22. For $M_{0}=2(d+2)+1$ and $s \geq 1+\left[M_{0}+\frac{d+1}{2}\right]$ let $G\left(x^{\prime}, \theta_{0}\right) \in H_{T}^{s+1}$ have compact support in $x^{\prime}$ and vanish in $t \leq 0$. Let $U_{\epsilon}\left(x, \theta_{0}\right) \in E_{T_{0}}^{s}$ be the exact solution to the singular system (1.26) for $0<\epsilon \leq \epsilon_{0}$ given by Theorem 1.13, let $\mathcal{V}^{0} \in \mathbb{H}_{T_{0}}^{s+1}$ be the profile given by Proposition 2.21, and let $\mathcal{U}^{0} \in \mathcal{P}_{T_{0}}^{s}$ be defined by

$$
\mathcal{U}^{0}\left(x, \theta_{0}, \xi_{d}\right)=\mathcal{V}^{0}\left(x, \theta_{0}+\underline{\omega}_{1} \xi_{d}, \ldots, \theta_{0}+\underline{\omega}_{M} \xi_{d}\right) .
$$

Here $0<T_{0} \leq T$ is the minimum of the existence times for the quasilinear problems (1.26) and (2.16). Define

$$
\mathcal{U}_{\epsilon}^{0}\left(x, \theta_{0}\right):=\mathcal{U}^{0}\left(x, \theta_{0}, \frac{x_{d}}{\epsilon}\right) .
$$

The family $\mathcal{U}_{\epsilon}^{0}$ is uniformly bounded in $E_{T_{0}}^{s}$ for $0<\epsilon \leq \epsilon_{0}$ and satisfies

$$
\left|U_{\epsilon}-\mathcal{U}_{\epsilon}^{0}\right|_{E_{T_{0}}^{s-1}} \rightarrow 0 \text { as } \epsilon \rightarrow 0 .
$$

The proof of Theorem 2.22 will use the strategy of simultaneous Picard iteration first used by Joly, Métivier, and Rauch in [8] to justify leading term expansions for initial value problems on domains without boundary. Consider the iteration schemes for the quasilinear problems (1.26) and (1.50):

a) $\partial_{x_{d}} U_{\epsilon}^{n+1}+\sum_{j=0}^{d-1} \tilde{A}_{j}\left(\epsilon U_{\epsilon}^{n}\right)\left(\partial_{x_{j}}+\frac{\beta_{j} \partial_{\theta_{0}}}{\epsilon}\right) U_{\epsilon}^{n+1}=F\left(\epsilon U_{\epsilon}^{n}\right) U_{\epsilon}^{n}$,

b) $\left.B\left(\epsilon U_{\epsilon}^{n}\right)\left(U_{\epsilon}^{n+1}\right)\right|_{x_{d}=0}=G\left(x^{\prime}, \theta_{0}\right)$,

c) $U_{\epsilon}^{n+1}=0$ in $t<0$,

and
a) $\mathbb{E} \mathcal{U}^{0, n+1}=\mathcal{U}^{0, n+1}$
b) $\mathbb{E}\left(\tilde{L}(\partial) \mathcal{U}^{0, n+1}+\mathcal{M}\left(\mathcal{U}^{0, n}\right) \partial_{\theta_{0}} \mathcal{U}^{0, n+1}\right)=\mathbb{E}\left(F(0) \mathcal{U}^{0, n}\right)$
c) $\left.B(0) \mathcal{U}^{0, n+1}\right|_{x_{d}=0, \xi_{d}=0}=G\left(x^{\prime}, \theta_{0}\right)$
d) $\mathcal{U}^{0, n+1}=0$ in $t<0$, 
where $\mathcal{U}^{0, n}\left(x, \theta_{0}, \xi_{d}\right):=\mathcal{V}^{0, n}\left(x, \theta_{0}+\underline{\omega}_{1} \xi_{d}, \ldots, \theta_{0}+\underline{\omega}_{M} \xi_{d}\right)$ for $\mathcal{V}^{0, n}$ as constructed in Proposition 2.19. Setting

$$
\mathcal{U}_{\epsilon}^{0, n}\left(x, \theta_{0}\right):=\mathcal{U}^{0, n}\left(x, \theta_{0}, \frac{x_{d}}{\epsilon}\right),
$$

we observe that to prove $(2.103)$ it suffices to prove boundedness of the family $\mathcal{U}_{\epsilon}^{0}$ in $E_{T_{0}}^{s}$ along with the following three statements:

(a) $\lim _{n \rightarrow \infty} U_{\epsilon}^{n}=U_{\epsilon}$ in $E_{T_{0}}^{s-1}$ uniformly with respect to $\epsilon \in\left(0, \epsilon_{0}\right]$

(b) $\lim _{n \rightarrow \infty} \mathcal{U}_{\epsilon}^{0, n}=\mathcal{U}_{\epsilon}^{0}$ in $E_{T_{0}}^{s-1}$ uniformly with respect to $\epsilon \in\left(0, \epsilon_{0}\right]$

(c) For each $n \lim _{\epsilon \rightarrow 0}\left|U_{\epsilon}^{n}-\mathcal{U}_{\epsilon}^{0, n}\right|_{E_{T_{0}}^{s-1}}=0$.

The first statement, together with uniform boundedness of the families $U_{\epsilon}^{n}, U_{\epsilon}$ in $E_{T_{0}}^{s}$, is proved in [23], Theorem 7.1 by showing convergence of the scheme (2.104) using the following linear estimate.

Proposition 2.23 ([23], Cor. 7.2). Let $s \geq\left[M_{0}+\frac{d+1}{2}\right]$ and consider the problem (2.104), where $G \in H_{T}^{s+1}$ has compact support and vanishes in $t \leq 0$, and where the right side of $(2.104)(a)$ is replaced by $\mathcal{F} \in \mathbb{E}_{T}^{s}$ with $\operatorname{supp} \mathcal{F} \subset\left\{t \geq 0,0 \leq x_{d} \leq E\right\}$. Suppose $U_{\epsilon}^{n} \in E_{T}^{s}$ has compact $x$-support and that for some $K>0, \epsilon_{1}>0$ we have

$$
\left|U_{\epsilon}^{n}\right|_{E_{T}^{s}}+\left|\epsilon \partial_{x_{d}} U_{\epsilon}^{n}\right|_{L^{\infty}} \leq K \text { for } \epsilon \in\left(0, \epsilon_{1}\right] .
$$

Then there exist constants $T_{0}(K)$ and $\epsilon_{0}(K) \leq \epsilon_{1}$ such that for $0<\epsilon \leq \epsilon_{0}$ and $T \leq T_{0}$ we have

$$
\left|U_{\epsilon}^{n+1}\right|_{E_{T}^{s}}+\sqrt{T}\left\langle U_{\epsilon}^{n+1}\right\rangle_{s+1, T} \leq C(K, E) \sqrt{T}\left(|\mathcal{F}|_{E_{T}^{s}}+\langle G\rangle_{s+1, T}\right) .
$$

Remark 2.24 (Finite propagation speed). By Assumption $1.6\left(\partial_{x_{d}}+\mathbb{A}\left(v, \partial_{x^{\prime}}\right), B(v)\right)$ is uniformly stable for $v \in \mathbb{R}^{N}$ with $|v| \leq R$ sufficiently small. A classical consequence of this and Assumptions 1.1 and 1.2 is that solutions $u$ vanishing in $t \leq 0$ to the boundary problem defined by $\left(\partial_{x_{d}}+\mathbb{A}\left(v, \partial_{x^{\prime}}\right), B(v)\right)$ for $|v| \leq R$ exhibit finite propagation speed (e.g., [3], Chapter 7). It is shown in [23], Proposition 7.1 that if $\alpha$ is an upper bound for the propagation speed of $\left(\partial_{x_{d}}+\mathbb{A}\left(v, \partial_{x^{\prime}}\right), B(v)\right)$ for $|v| \leq R$, then $\alpha$ is also an upper bound for the propagation speed of solutions to (2.104) when $\left|\epsilon U_{\epsilon}^{n}\right|_{L^{\infty}}<R$.

The boundedness of $\mathcal{U}_{\epsilon}^{0}$ in $E_{T_{0}}^{s}$ and (2.107)(b) follow from the next Lemma.

Lemma 2.25. (a) For $m \geq 0$ suppose $\mathcal{V}(x, \theta) \in \mathbb{H}_{T}^{m+1}, \mathbb{E}^{\prime} \mathcal{V}=\mathcal{V}$, and set $\mathcal{U}\left(x, \theta_{0}, \xi_{d}\right)=$ $\mathcal{V}\left(x, \theta_{0}+\underline{\omega}_{1} \xi_{d}, \ldots, \theta_{0}+\underline{\omega}_{M} \xi_{d}\right)$. Then

$$
|\mathcal{U}|_{\mathcal{E}_{T}^{m}} \leq C|\mathcal{V}|_{\mathbb{H}_{T}^{m+1}}
$$

(b) For $m \geq 0$ and $\mathcal{U}$ as in (a), $\operatorname{set} \mathcal{U}_{\epsilon}\left(x, \theta_{0}\right)=\mathcal{U}\left(x, \theta_{0}, \frac{x_{d}}{\epsilon}\right)$. Then

$$
\left|\mathcal{U}_{\epsilon}\right|_{E_{T}^{m}} \leq|\mathcal{U}|_{\mathcal{E}_{T}^{m}}
$$


Proof. a. Since $\mathbb{E}^{\prime} \mathcal{V}=\mathcal{V}, \mathcal{V}$ has an expansion of the form (2.19). Thus, (2.110) follows from Lemma 2.7.

b. Clearly,

$$
\left|\mathcal{U}_{\epsilon}\left(x, \theta_{0}\right)\right|_{C\left(x_{d}, H^{s}\left(x^{\prime}, \theta_{0}\right)\right)} \leq \sup _{\xi_{d}}\left|\mathcal{U}\left(x^{\prime}, x_{d}, \theta_{0}, \xi_{d}\right)\right|_{C\left(x_{d}, H_{T}^{s}\left(x^{\prime}, \theta_{0}\right)\right)} .
$$

Since $\mathbb{E}^{\prime} \mathcal{V}=\mathcal{V}$, to finish it suffices to consider $\tilde{f}\left(x, \theta_{0}, \xi_{d}\right)=f\left(x, \theta_{0}+\underline{\omega}_{j} \xi_{d}\right)$ for $f\left(x, \theta_{j}\right) \in$ $H_{T}^{s+1}\left(x, \theta_{j}\right)$ and show that for any $\xi_{d}$ :

$$
\left|\tilde{f}\left(x^{\prime}, x_{d}, \theta_{0}, \frac{x_{d}}{\epsilon}\right)\right|_{L^{2}\left(x_{d}, H_{T}^{s+1}\left(x^{\prime}, \theta_{0}\right)\right)}^{2}=\left|\tilde{f}\left(x^{\prime}, x_{d}, \theta_{0}, \xi_{d}\right)\right|_{L^{2}\left(x_{d}, H_{T}^{s+1}\left(x^{\prime}, \theta_{0}\right)\right)}^{2} .
$$

Using (2.27) and (2.28) we see that each side of (2.113) is equal to $\left|f\left(x^{\prime}, x_{d}, \theta_{j}\right)\right|_{L^{2}\left(x_{d}, H_{T}^{s+1}\right)}^{2}$

Proof of Theorem 2.22. 1. The boundedness of the families $U_{\epsilon}^{n}$ and $U_{\epsilon}$ in $E_{T_{0}}^{s}$ and the statement (2.107)(a) were proved in [23], Theorem 7.1.

2. Since the family $\mathcal{V}^{0, n}$ is bounded in $\mathbb{H}_{T_{0}}^{s+1}$ and converges to $\mathcal{V}^{0}$ in $\mathbb{H}_{T_{0}}^{s}$, the boundedness of $\mathcal{U}_{\epsilon}^{0, n}$ in $E_{T_{0}}^{s}$ and the limit (2.107)(b) follow directly from (2.110) and (2.111).

3. Strategy for proving $(2.107)(\mathbf{c})$. Assuming that (2.107)(c) holds for $n$, we will show that it holds for $n+1$. One could prove this using the estimate (2.109) if

$$
\begin{aligned}
& \text { (a) }\left|\left(\partial_{x_{d}}+\mathbb{A}\left(\epsilon U_{\epsilon}^{n}, \partial_{x^{\prime}}+\frac{\beta \cdot \partial_{\theta_{0}}}{\epsilon}\right)\right)\left(U_{\epsilon}^{n+1}-\mathcal{U}_{\epsilon}^{0, n+1}\right)\right|_{E_{T_{0}}^{s-1}} \rightarrow 0 \text { as } \epsilon \rightarrow 0 \\
& \text { (b) }\left.\left|B\left(\epsilon U_{\epsilon}^{n}\right)\left(U_{\epsilon}^{n+1}-\mathcal{U}_{\epsilon}^{0, n+1}\right)\right|_{x_{d}=0}\right|_{H_{T_{0}}^{s}} \rightarrow 0 \text { as } \epsilon \rightarrow 0 .
\end{aligned}
$$

It is easy to check (see below) that (2.105)(c) implies (2.114)(b), but the norm in (2.114)(a) is only $O(1)$ as $\epsilon \rightarrow 0$ as a consequence of (2.105)(a), and remains $O(1)$ even if (2.105)(b) is taken into account. Thus, it is natural to seek a corrector $\mathcal{U}^{1}$ similar to the one introduced earlier in (1.42) and satisfying a condition like (1.46). As we saw in Lemma 1.17, in such an argument the profile equation (2.105)(b) should play the role of a solvability condition. Difficulties with small divisors (see Remark 2.26) force us to approximate $\mathcal{U}^{0, n}$ and $\mathcal{U}^{0, n+1}$ by trigonometric polynomials $\mathcal{U}_{p}^{0, n}$ and $\mathcal{U}_{p}^{0, n+1}$ before constructing a corrector $\mathcal{U}_{p}^{1}$, and to prove estimates weaker than (2.114). Fortunately, these estimates will be strong enough to imply $(2.107)$ (c) for $n+1$.

4. Fix $\delta>0$. Since $\mathbb{E} \mathcal{U}^{0, n}=\mathcal{U}^{0, n}$ and $\mathbb{E} \mathcal{U}^{0, n+1}=\mathcal{U}^{0, n+1}$, we can use Remark 2.8 to choose trigonometric polynomials $\mathcal{U}_{p}^{0, n}$ and $\mathcal{U}_{p}^{0, n+1}$ such that

(a) $\mathbb{E} \mathcal{U}_{p}^{0, n}=\mathcal{U}_{p}^{0, n}$ and $\mathbb{E} \mathcal{U}_{p}^{0, n+1}=\mathcal{U}_{p}^{0, n+1}$,

(b) $\left|\mathcal{U}^{0, n}-\mathcal{U}_{p}^{0, n}\right|_{\mathcal{E}_{T_{0}}^{s}}<\delta,\left|\mathcal{U}^{0, n+1}-\mathcal{U}_{p}^{0, n+1}\right|_{\mathcal{E}_{T_{0}}^{s}}<\delta$, and $\left|\partial_{x_{d}} \mathcal{U}^{0, n+1}-\partial_{x_{d}} \mathcal{U}_{p}^{0, n+1}\right|_{\mathcal{E}_{T_{0}}^{s-1}}<\delta$. 
We smooth the coefficients of $\mathcal{U}_{p}^{0, n}$ and $\mathcal{U}_{p}^{0, n+1}$ to be $H^{\infty}$ and so that $(2.115)$ still holds. (2.115).

The boundedness of the family $U_{\epsilon}^{n}$ in $E_{T_{0}}^{s}$ together with the induction assumption

$$
\left|U_{\epsilon}^{n}-\mathcal{U}_{\epsilon}^{0, n}\right|_{E_{T_{0}}^{s-1}} \rightarrow 0 \text { as } \epsilon \rightarrow 0
$$

imply

$$
\left|F\left(\epsilon U_{\epsilon}^{n}\right)\left(U_{\epsilon}^{n}\right)-F(0) \mathcal{U}_{\epsilon}^{0, n}\right|_{E_{T_{0}}^{s-1}} \rightarrow 0 \text { as } \epsilon \rightarrow 0 .
$$

In view of (2.115) and (2.111) this implies

$$
\left|F\left(\epsilon U_{\epsilon}^{n}\right)\left(U_{\epsilon}^{n}\right)-F(0) \mathcal{U}_{p, \epsilon}^{0, n}\right|_{E_{T_{0}}^{s-1}} \leq C \delta+c(\epsilon),
$$

where $c(\epsilon) \rightarrow 0$ as $\epsilon \rightarrow 0$.

5. Define

$$
\mathcal{G}_{p}=\tilde{L}\left(\partial_{x}\right) \mathcal{U}_{p}^{0, n+1}+\mathcal{M}\left(\mathcal{U}_{p}^{0, n}\right) \partial_{\theta_{0}} \mathcal{U}_{p}^{0, n+1}
$$

We claim that

$$
\left|\mathbb{E} \mathcal{G}_{p}-\mathbb{E}\left(F(0) \mathcal{U}_{p}^{0, n}\right)\right|_{\mathcal{E}_{T_{0}}^{s-1}}=O(\delta) .
$$

Indeed, from $(2.115)(\mathrm{b})$ and the continuity of $\mathbb{E}$ on $\mathcal{P}^{t}$ spaces (Prop. 2.2) we have

$$
\left|\mathbb{E}\left(F(0) \mathcal{U}^{0, n}-F(0) \mathcal{U}_{p}^{0, n}\right)\right|_{\mathcal{E}_{T_{0}}^{s}}=O(\delta) .
$$

But $\mathbb{E}\left(F(0) \mathcal{U}^{0, n}\right)$ is given by the left side of $(2.105)$ (b), so (2.120) follows by observing that (2.115)(b) and Proposition 2.2(a) imply

$$
\begin{aligned}
& \left|\tilde{L}\left(\partial_{x}\right)\left(\mathcal{U}^{0, n+1}-\mathcal{U}_{p}^{0, n+1}\right)\right|_{\mathcal{E}_{T_{0}}^{s-1}}=O(\delta) \\
& \left|\mathcal{M}\left(\mathcal{U}^{0, n}\right) \partial_{\theta_{0}} \mathcal{U}^{0, n+1}-\mathcal{M}\left(\mathcal{U}_{p}^{0, n}\right) \partial_{\theta_{0}} \mathcal{U}_{p}^{0, n+1}\right|_{\mathcal{E}_{T_{0}}^{s-1}}=O(\delta) .
\end{aligned}
$$

6. Next define the operator

$$
\mathbb{L}_{0}=\tilde{L}\left(\partial_{x}\right)+\frac{1}{\epsilon} \tilde{L}\left(d \phi_{0}\right) \partial_{\theta_{0}}+\mathcal{M}\left(\mathcal{U}_{p, \epsilon}^{0, n}\right) \partial_{\theta_{0}},
$$

which is an approximation to the operator appearing on the left side of (2.104)(a) that will allow us to use Lemma 1.17 to construct a corrector $\mathcal{U}_{p}^{1}$. Indeed, we claim

$$
\left|\mathbb{L}_{0} U_{\epsilon}^{n+1}-F\left(\epsilon U_{\epsilon}^{n}\right) U_{\epsilon}^{n}\right|_{E_{T_{0}}^{s-1}} \leq C \delta+c(\epsilon),
$$


where $c(\epsilon) \rightarrow 0$ as $\epsilon \rightarrow 0$. This follows from (2.104)(a) and

$$
\begin{aligned}
& \left|\tilde{A}_{j}\left(\epsilon U_{\epsilon}^{n}\right) \partial_{x_{j}} U_{\epsilon}^{n+1}-\tilde{A}_{j}(0) \partial_{x_{j}} U_{\epsilon}^{n+1}\right|_{E_{T_{0}}^{s-1}}=O(\epsilon) \\
& \left|\frac{1}{\epsilon} \tilde{A}_{j}\left(\epsilon U_{\epsilon}^{n}\right) \beta_{j} \partial_{\theta_{0}} U_{\epsilon}^{n+1}-\left(\frac{1}{\epsilon} \tilde{A}_{j}(0) \beta_{j} \partial_{\theta_{0}} U_{\epsilon}^{n+1}+\partial_{u} \tilde{A}_{j}(0) U_{\epsilon}^{n} \beta_{j} \partial_{\theta_{0}} U_{\epsilon}^{n+1}\right)\right|_{E_{T_{0}}^{s-1}}=O(\epsilon) \\
& \left|\partial_{u} \tilde{A}_{j}(0)\left(U_{\epsilon}^{n}-\mathcal{U}_{p, \epsilon}^{0, n}\right) \beta_{j} \partial_{\theta_{0}} U_{\epsilon}^{n+1}\right|_{E_{T_{0}}^{s-1}} \leq C\left|U_{\epsilon}^{n}-\mathcal{U}_{p, \epsilon}^{0, n}\right|_{E_{T_{0}}^{s-1}} \leq c(\epsilon)+O(\delta) .
\end{aligned}
$$

7. Construction of the corrector. First observe that since $\tilde{\mathcal{L}}\left(\partial_{\theta_{0}}, \partial_{\xi_{d}}\right) \mathcal{U}_{p}^{0, n+1}=0$ we have

$$
\mathbb{L}_{0} \mathcal{U}_{p, \epsilon}^{0, n+1}=\mathcal{G}_{p, \epsilon}
$$

and thus

$$
\begin{aligned}
& \mathbb{L}_{0} \mathcal{U}_{p, \epsilon}^{0, n+1}-F(0) \mathcal{U}_{p, \epsilon}^{0, n}=\mathcal{G}_{p, \epsilon}-F(0) \mathcal{U}_{p, \epsilon}^{0, n}= \\
& \quad\left(\mathbb{E}\left(\mathcal{G}_{p}-F(0) \mathcal{U}_{p}^{0, n}\right)\right)_{\epsilon}+\left((I-\mathbb{E})\left(\mathcal{G}_{p}-F(0) \mathcal{U}_{p}^{0, n}\right)\right)_{\epsilon} .
\end{aligned}
$$

The first term on the right is $O(\delta)$ in $E_{T_{0}}^{s-1}$ by (2.120) and (2.111), but the second term is not small, so we construct $\mathcal{U}_{p}^{1}$ to solve (most of) it away. By Lemma 1.17 there is a trigonometric polynomial $\mathcal{U}_{p}^{1} \in \mathcal{E}_{T_{0}}^{s}$ such that

$$
\tilde{\mathcal{L}}\left(\partial_{\theta_{0}}, \partial_{\xi_{d}}\right) \mathcal{U}_{p}^{1}=-(I-\mathbb{E})\left(\mathcal{G}_{p}-F(0) \mathcal{U}_{p}^{0, n}\right) .
$$

We have

$$
\left|\mathbb{L}_{0}\left(\mathcal{U}_{p, \epsilon}^{0, n+1}+\epsilon \mathcal{U}_{p, \epsilon}^{1}\right)-F(0) \mathcal{U}_{p, \epsilon}^{0, n}\right|_{E_{T_{0}}^{s-1}} \leq C \delta+K(\delta) \epsilon,
$$

This is a consequence of $(2.127),(2.128)$, and

$$
\mathbb{L}_{0}\left(\epsilon \mathcal{U}_{p, \epsilon}^{1}\right)=\left(\tilde{\mathcal{L}}\left(\partial_{\theta_{0}}, \partial_{\xi_{d}}\right) \mathcal{U}_{p}^{1}\right)_{\epsilon}+\tilde{L}(\partial)\left(\epsilon \mathcal{U}_{p, \epsilon}^{1}\right)+\mathcal{M}\left(\mathcal{U}_{p, \epsilon}^{0, n}\right) \partial_{\theta_{0}}\left(\epsilon \mathcal{U}_{p, \epsilon}^{1}\right)
$$

since the sum of the last two terms on the right is $\leq K(\delta) \epsilon$ in $E_{T_{0}}^{s-1}$. Using (2.118), (2.124), and (2.129), we obtain

$$
\left|\mathbb{L}_{0}\left(U_{\epsilon}^{n+1}-\left(\mathcal{U}_{p, \epsilon}^{0, n+1}+\epsilon \mathcal{U}_{p, \epsilon}^{1}\right)\right)\right|_{E_{T_{0}}^{s-1}} \leq C \delta+c(\epsilon)+K(\delta) \epsilon,
$$

where $c(\epsilon) \rightarrow 0$ as $\epsilon \rightarrow 0$ and $C$ is independent of $\delta$ and $\epsilon$.

8. Instead of (2.114) we claim that the following estimates hold:

(a) $\left|\left(\partial_{x_{d}}+\mathbb{A}\left(\epsilon \mathcal{U}_{p, \epsilon}^{0, n}, \partial_{x^{\prime}}+\frac{\beta \cdot \partial_{\theta_{0}}}{\epsilon}\right)\right)\left(U_{\epsilon}^{n+1}-\left(\mathcal{U}_{p, \epsilon}^{0, n+1}+\epsilon \mathcal{U}_{p, \epsilon}^{1}\right)\right)\right|_{E_{T_{0}}^{s-1}} \leq C \delta+c(\epsilon)+K(\delta) \epsilon$
$(b)\left|B\left(\epsilon \mathcal{U}_{p, \epsilon}^{0, n}\right)\left(U_{\epsilon}^{n+1}-\left(\mathcal{U}_{p, \epsilon}^{0, n+1}+\epsilon \mathcal{U}_{p, \epsilon}^{1}\right)\right)\right|_{H_{T_{0}}^{s}} \leq C \delta+c(\epsilon)+K(\delta) \epsilon$, 
where $c(\epsilon) \rightarrow 0$ as $\epsilon \rightarrow 0$ and $C$ is independent of $\delta$ and $\epsilon$. Indeed, (2.132)(a) follows from (2.131) by estimates similar to (2.125), while (2.132)(b) is a simple consequence of (2.104)(b) and (2.105)(c). Applying Proposition 2.23 we find

$$
\left|U_{\epsilon}^{n+1}-\left(\mathcal{U}_{p, \epsilon}^{0, n+1}+\epsilon \mathcal{U}_{p, \epsilon}^{1}\right)\right|_{E_{T_{0}}^{s-1}} \leq C \delta+c(\epsilon)+K(\delta) \epsilon,
$$

and thus

$$
\left|U_{\epsilon}^{n+1}-\mathcal{U}_{\epsilon}^{0, n+1}\right|_{E_{T_{0}}^{s-1}} \leq C \delta+c(\epsilon)+K(\delta) \epsilon .
$$

This completes the induction step and the proof of Theorem 2.22.

Remark 2.26. (Small divisors). If the right side of (2.128), call it $\mathcal{H}\left(x, \theta_{0}, \xi_{d}\right)$, were an infinite trigonometric series instead of a trigonometric polynomial, one could still solve the equations

$$
i \tilde{L}\left(\kappa_{0} \beta, \kappa_{d}\right) U_{\kappa}^{1}(x)=H_{\kappa}(x), \quad \kappa=\left(\kappa_{0}, \kappa_{d}\right) \in \mathbb{Z} \times \mathbb{R},
$$

where $H_{\kappa}$ denotes a Fourier coefficient of $\mathcal{H}$. However, the possibility of there being infinitely many noncharacteristic modes $\kappa \notin \mathcal{C}$ for which $\operatorname{det} \tilde{L}\left(\kappa_{0} \beta, \kappa_{d}\right) \sim 0$ generally prevents convergence of $\mathcal{U}\left(x, \theta_{0}, \xi_{d}\right)=\sum_{\kappa} U_{\kappa}(x) e^{i \kappa_{0} \theta_{0}+i \kappa_{d} \xi_{d}}$ in any reasonable space. This is why it is not always possible to construct higher than leading order geometric optics expansions.

\section{A free boundary problem: highly oscillatory shocks}

Consider the system of conservation laws

$$
\sum_{j=0}^{d} \partial_{x_{j}} f_{j}(u)=0
$$

on $\mathbb{R}^{d+1}$, where the $f_{j}: \mathbb{R}^{N} \rightarrow \mathbb{R}^{N}$ are $C^{\infty}$ functions. Set $x=\left(x^{\prime}, x_{d}\right)=\left(t, y, x_{d}\right)$ and let $S$ be a noncharacteristic surface for (3.1) defined by $x_{d}=\psi\left(x^{\prime}\right)$, where $\psi$ is $C^{1}$. Suppose $u$ is a $C^{1}$ function up to $S$ on each side of $S$ whose restrictions $u^{ \pm}$to $\pm\left(x_{d}-\psi\left(x^{\prime}\right)\right)>0$ satisfy (3.1). Then $u$ is a multidimensional shock if, in addition, $u^{ \pm}$satisfy the jump condition

$$
\sum_{j=0}^{d-1} \psi_{x_{j}}\left[f_{j}(u)\right]-\left[f_{d}(u)\right]=0 \text { on } S .
$$

Functions $u$ as above satisfying (3.1) on each side of $S$ are weak solutions of (3.1) in $\mathbb{R}^{d+1}$ if and only if (3.2) holds. Equations (3.1) and (3.2) constitute a hyperbolic free boundary problem for the unknowns $\left(u^{ \pm}, \psi\right)$. This problem was solved by Majda [13] under an appropriate stability hypothesis, with several improvements by Metivier [16, 18] . 
We reduce to a problem with fixed boundary by making the change of variables $\tilde{x}^{\prime}=x^{\prime}$, $\tilde{x}_{d}=x_{d}-\psi\left(x^{\prime}\right)$, and putting $\tilde{u}^{ \pm}(\tilde{x})=u^{ \pm}(x)$. Setting

$$
\begin{aligned}
& A_{j}(v)=f_{j}^{\prime}(v), j=0, \ldots, d \\
& \mathcal{A}_{d}\left(v, d_{x^{\prime}} \psi\right)=A_{d}(v)-\sum_{j=0}^{d-1} \psi_{x_{j}} A_{j}(v)
\end{aligned}
$$

and dropping the tildes, we obtain the equations

$$
\begin{gathered}
P^{ \pm}\left(u^{ \pm}, d_{x^{\prime}} \psi\right) \partial_{x} u^{ \pm}=\sum_{j=0}^{d-1} A_{j}\left(u^{ \pm}\right) \partial_{x_{j}} u^{ \pm}+\mathcal{A}_{d}\left(u^{ \pm}, d_{x^{\prime}} \psi\right) \partial_{x_{d}} u^{ \pm}=0 \text { on } \pm x_{d}>0 \\
G\left(u^{ \pm}, d_{x^{\prime}} \psi\right)=\sum_{j=0}^{d-1} \psi_{x_{j}}\left[f_{j}(u)\right]-\left[f_{d}(u)\right]=0 \text { on } x_{d}=0 .
\end{gathered}
$$

We will study oscillatory perturbations of a planar shock solution $\left(\underline{u}^{ \pm}, \sigma x_{0}\right)$ of (3.4) and (3.5). A planar shock is given by constants $\left(\underline{u}^{ \pm}, \sigma\right)$ satisfying

$$
\sigma\left[f_{0}(\underline{u})\right]-\left[f_{d}(\underline{u})\right]=0 .
$$

\subsection{Assumptions.}

The assumptions we make for shocks are small modifications of those made in the fixed boundary case. We set

$$
\bar{A}_{j}(u)=A_{0}(u)^{-1} A_{j}(u) \text { for } j=1, \ldots, d
$$

and make the exact analogue of Assumption 1.1 where (1.10) is replaced by

$$
\operatorname{det}\left[\tau I+\sum_{j=1}^{d} \xi_{j} \bar{A}_{j}\left(u^{ \pm}\right)\right]=\prod_{k=1}^{q^{ \pm}}\left(\tau+\lambda_{k}^{ \pm}\left(u^{ \pm}, \xi\right)\right)^{\nu_{k}^{ \pm}}
$$

and $u^{ \pm} \in \mathcal{O}^{ \pm}$, a neighborhood of $\underline{u}^{ \pm}$. In place of Assumption 1.2 we assume that $\mathcal{A}_{d}\left(u^{ \pm}, \omega\right)$ is invertible for $u^{ \pm} \in \mathcal{O}^{ \pm}$and $\omega \in \mathbb{R}^{d}$ near $d\left(\sigma x_{0}\right)$. We define the hyperbolic regions $\mathcal{H}^{ \pm}$as in Definition 1.3, where now

$$
\mathcal{A}^{ \pm}(\zeta):=-i \mathcal{A}_{d}^{-1}\left(\underline{u}^{ \pm}, d_{x^{\prime}}\left(\sigma x_{0}\right)\right)\left((\tau-i \gamma) A_{0}(\underline{u})+\sum_{j=1}^{d-1} \eta_{j} A_{j}(\underline{u})\right) .
$$

For $\zeta \in \Xi \backslash \Xi_{0}$ let $\mathbb{E}^{+}(\zeta)$ denote the stable subspace of $\mathcal{A}^{+}(\zeta)$ and $\mathbb{E}^{-}(\zeta)$ the unstable subspace of $\mathcal{A}^{-}(\zeta)$; and use the same notation for their continuous extensions to $\Xi$. Assumption 1.6 is replaced by the assumption that the planar shock $\left(\underline{u}^{ \pm}, \sigma x_{0}\right)$ is uniformly 
stable in the sense of Majda [13]. To define this consider the linearization of (3.5) about the planar shock:

$$
B\left(\underline{u}^{ \pm}, d_{x^{\prime}}\left(\sigma x_{0}\right)\right)\left(v^{ \pm}, d_{x^{\prime}} \beta\right):=\sum_{j=0}^{d-1} \beta_{x_{j}}\left[f_{j}(\underline{u})\right]-\left[\mathcal{A}_{d}\left(\underline{u}, d_{x^{\prime}}\left(\sigma x_{0}\right)\right) v\right] .
$$

Definition 3.1. The planar shock $\left(\underline{u}^{ \pm}, \sigma x_{0}\right)$ is uniformly stable if and only if for all $\zeta \in \Xi$ the map

$$
\begin{aligned}
& \left(v^{+}, v^{-}, \lambda\right) \rightarrow B\left(\underline{u}^{ \pm}, d_{x^{\prime}}\left(\sigma x_{0}\right)\right)\left(v^{ \pm}, \lambda \zeta\right)= \\
& \quad \lambda\left((\tau-i \gamma)\left[f_{0}(\underline{u})\right]+\sum_{j=1}^{d-1} \eta_{j}\left[f_{j}(\underline{u})\right]\right)-\left[\mathcal{A}_{d}\left(\underline{u}, d_{x^{\prime}}\left(\sigma x_{0}\right)\right) v\right]
\end{aligned}
$$

is an isomorphism from $\mathbb{E}^{+}(\zeta) \times \mathbb{E}^{-}(\zeta) \times \mathbb{C}$ onto $\mathbb{C}^{N}$.

The uniform stability condition implies that $\operatorname{dim}\left(\mathbb{E}^{+}(\zeta) \times \mathbb{E}^{-}(\zeta)\right)=N-1$, and therefore that $\left(\underline{u}, \sigma x_{0}\right)$ is a Lax shock.

Next we define the boundary and interior phases. In place of (1.12) we set

$$
L^{ \pm}\left(\partial_{x}\right)=\partial_{t}+\sum_{j=1}^{d-1} \bar{A}_{j}\left(\underline{u}^{ \pm}\right) \partial_{x_{j}}+\overline{\mathcal{A}}_{d}\left(\underline{u}^{ \pm}, d_{x^{\prime}}\left(\sigma x_{0}\right)\right) \partial_{x_{d}}
$$

and define $\tilde{\lambda}_{j}^{ \pm}(\xi)$ by

$$
\operatorname{det} L^{ \pm}(\tau, \xi)=\prod_{k=1}^{q^{ \pm}}\left(\tau+\tilde{\lambda}_{k}^{ \pm}(\xi)\right)^{\nu_{k}^{ \pm}}
$$

In place of Assumption 1.8 we now fix once and for all

$$
\beta=(\underline{\tau}, \underline{\eta}) \in \mathcal{H}^{+} \cap \mathcal{H}^{-}
$$

and define the boundary phase $\phi_{0}\left(x^{\prime}\right)=\beta \cdot x^{\prime} .{ }^{8}$ Let $\underline{\omega}_{m}^{ \pm}, m=1, \ldots, M^{ \pm}$, be the distinct roots of the dispersion relation

$$
\operatorname{det} L^{ \pm}(\underline{\tau}, \underline{\eta}, \omega)=0 \text {. }
$$

To each (necessarily real) root $\omega_{m}^{ \pm}$there corresponds a unique $k_{m}^{ \pm} \in\left\{1, \ldots, q^{ \pm}\right\}$such that $\underline{\tau}+\tilde{\lambda}_{k_{m}^{ \pm}}^{ \pm}\left(\underline{\eta}, \underline{\omega}_{m}^{ \pm}\right)=0$

\footnotetext{
${ }^{8}$ The intersection in (3.14) is nonempty since it contains a neighborhood of $(1,0)$, for example.
} 
Definition 3.2. We define real characteristic phases

$$
\phi_{m}^{ \pm}(x)=\beta \cdot x^{\prime}+\underline{\omega}_{m}^{ \pm} x_{d}, m=1, \ldots, M^{ \pm} .
$$

The phase $\phi_{m}^{+}$is said to be incoming to $\left\{x_{d}>0\right\}$ if $\partial_{\xi_{d}} \tilde{\lambda}_{k_{m}^{+}}^{+}\left(\underline{\eta}, \underline{\omega}_{m}^{+}\right)>0$ and to be outgoing from $\left\{x_{d}>0\right\}$ if $\left.\partial_{\xi_{d}} \tilde{\lambda}_{k_{m}^{+}}^{+} \underline{\eta}, \underline{\omega}_{m}^{+}\right)<0$. Phases $\phi_{m}^{-}$incoming to $x_{d}<0$ and outgoing from $x_{d}<0$ are defined similarly (but with signs reversed on the derivatives). We let

$$
\left\{\phi_{m}^{ \pm}, m \in \mathbb{I}\right\}, \text { resp. }\left\{\phi_{m}^{ \pm}, m \in \mathbb{O}\right\}
$$

denote the sets of phases that are incoming to either $x_{d}>0$ or $x_{d}<0$, respectively outgoing from either $x_{d}>0$ or $x_{d}<0$.

The kernel of $L^{ \pm}\left(d \phi_{m}^{ \pm}\right)$has dimension $\nu_{k_{m}}^{ \pm}$and for each $m \in\left\{1, \ldots, M^{ \pm}\right\}$we let

$$
r_{m, k}^{ \pm}, k=1 \ldots, \nu_{k_{m}}^{ \pm}
$$

denote a basis of ker $L^{ \pm}\left(d \phi_{m}^{ \pm}\right)$consisting of real vectors. We let $P_{m}^{ \pm}, m=1, \ldots, M^{ \pm}$, denote the projectors associated to the decompositions

$$
\mathbb{C}^{N}=\oplus_{m=1}^{M^{ \pm}} \operatorname{ker} L^{ \pm}\left(d \phi_{m}^{ \pm}\right) .
$$

Example 3.3 (Euler shocks). Consider again the $3 D$ isentropic, compressible Euler equations in variables $(\rho, u)$, a planar shock $\left(\underline{\rho}^{ \pm}, \underline{u}^{ \pm}\right), x_{3}=\sigma x_{0}$, and define $\lambda_{k}(\rho, u, \xi)$ as in (1.15). The functions $\tilde{\lambda}_{j}^{ \pm}(\xi)$ as in (3.13) are

$$
\tilde{\lambda}_{j}^{ \pm}(\xi)=\lambda_{j}\left(\underline{\rho}^{ \pm}, \underline{u}^{ \pm}, \xi\right)-\sigma \xi_{3}, j=1,2,3 \text {, where } \nu_{1}^{ \pm}=\nu_{3}^{ \pm}=1, \nu_{2}^{ \pm}=2 .
$$

The noncharacteristic condition is, with $e_{3}=(0,0,1)$, the condition $\tilde{\lambda}_{j}^{ \pm}\left(e_{3}\right) \neq 0, j=$ $1,2,3$, or equivalently:

$$
\underline{u}_{3}^{+}-\sigma \notin\left\{0, c^{+},-c^{+}\right\} \text {and } \underline{u}_{3}^{-}-\sigma \notin\left\{0, c^{-},-c^{-}\right\} \text {, where } c^{ \pm}=\sqrt{p^{\prime}\left(\underline{\rho}^{ \pm}\right)} .
$$

Euler shocks are always extreme shocks, that is, they are always associated to either the smallest eigenvalue $\lambda_{1}$ or the largest $\lambda_{3}$ (a 1-shock or 3-shock, respectively). For example, a planar 3 -shock $\left(\underline{u}^{ \pm}, \sigma x_{0}\right)$ satisfies

$$
\begin{aligned}
& \tilde{\lambda}_{3}^{+}\left(e_{3}\right)<0<\tilde{\lambda}_{3}^{-}\left(e_{3}\right), \quad \tilde{\lambda}_{2}^{-}\left(e_{3}\right)<0, \quad \text { that is, } \\
& \underline{u}_{3}^{+}+c^{+}-\sigma<0<\underline{u}_{3}^{-}+c^{-}-\sigma, \quad \underline{u}_{3}^{-}-\sigma<0 .
\end{aligned}
$$

This implies

$$
\left|\underline{u}_{3}^{+}-\sigma\right|>c^{+} \text {and }\left|\underline{u}_{3}^{-}-\sigma\right|<c_{-},
$$


so the relative normal speed changes from supersonic to subsonic (and density increases) as fluid passes from the + to the - side. The hyperbolic region is $\mathcal{H}=\mathcal{H}^{+} \cap \mathcal{H}^{-}=\mathcal{H}^{-}$, since in this case

$$
\mathcal{H}^{-}=\left\{(\tau, \eta) \in \mathbb{R}^{3}:\left|\tau+\underline{u}_{1}^{-} \eta_{1}+\underline{u}_{2}^{-} \eta_{2}\right|>\sqrt{\left(c^{-}\right)^{2}-\left(\underline{u}_{3}^{-}-\sigma\right)^{2}}|\eta|\right\} \text { and } \mathcal{H}^{+}=\mathbb{R}^{3} \backslash 0 .
$$

For a given $\phi_{0}=\beta \cdot x^{\prime}$ with $\beta \in \mathcal{H}$, one can now easily construct triples of resonant phases (containing $\phi_{0}$ or not) on either the + or - side as in Example 1.18.

In Majda [13], Chapter 3, explicit criteria for Euler shocks to be uniformly stable are given in terms of flow quantities (Mach numbers, compression ratios, etc.). In particular, for isentropic flow of an ideal gas with equation of state $p(\rho)=A \rho^{\gamma}, \gamma>1$, all shocks are uniformly stable. The criteria imply also that for nonisentropic flow of an ideal gas with equation of state $p(\rho, S)=\exp \left(\frac{S}{c_{v}}\right) \rho^{\gamma}, \gamma>1$, all shocks are uniformly stable.

Remark 3.4. We will refer to assumptions made for shocks using the labels of their fixed boundary analogues. So, for example, Shock Assumption 1.1 now refers to the modification described above of the fixed boundary Assumption 1.1. Shock Assumptions 1.1, 1.2, 1.6, 1.8 are in force throughout the remainder of the paper.

\subsection{The singular shock problem}

The perturbed shock $\left(u_{\epsilon}^{ \pm}, \psi_{\epsilon}\right)$ is the solution to an initial-transmission problem on $\mathbb{R}^{d+1}$ with oscillatory initial data:

$$
\begin{aligned}
& \text { (a) } P^{ \pm}\left(u_{\epsilon}^{ \pm}, d_{x^{\prime}} \psi_{\epsilon}\right) \partial_{x} u_{\epsilon}^{ \pm}=0 \text { in } \pm x_{d}>0 \\
& \text { (b) } G\left(u_{\epsilon}^{ \pm}, d_{x^{\prime}} \psi_{\epsilon}\right)=0 \text { on } x_{d}=0 \\
& \text { (c) } u_{\epsilon}^{ \pm}=\underline{u}^{ \pm}+\left.\epsilon w_{\epsilon}^{ \pm}\left(x^{\prime \prime}, x_{d}, \theta_{0}\right)\right|_{\theta_{0}=\frac{\beta x^{\prime}}{\epsilon}} \text { on } x_{0}=0 \\
& \text { (d) } \psi_{\epsilon}=\sigma x_{0}+\left.\epsilon \chi_{\epsilon}\left(x, \theta_{0}\right)\right|_{\theta_{0}=\frac{\beta x^{\prime}}{\epsilon}} \text { with } \chi_{\epsilon}=0 \text { on } x_{0}=0
\end{aligned}
$$

where $w_{\epsilon}^{ \pm}\left(x^{\prime \prime}, x_{d}, \theta_{0}\right)$ and $\chi_{\epsilon}\left(x^{\prime}, \theta_{0}\right)$ satisfy appropriate corner and phase compatibility conditions, and $w_{\epsilon}^{ \pm}$is chosen to introduce oscillations that reflect transversally off the shock.

One can look for solutions to (3.25) of the form

$$
\begin{aligned}
& u_{\epsilon}^{ \pm}(x)=\left.\mathbb{U}_{\epsilon}^{ \pm}\left(x, \theta_{0}\right)\right|_{\theta_{0}=\frac{\beta x^{\prime}}{\epsilon}} \\
& \psi_{\epsilon}\left(x^{\prime}\right)=\left.\Psi_{\epsilon}\left(x^{\prime}, \theta_{0}\right)\right|_{\theta_{0}=\frac{\beta x^{\prime}}{\epsilon}},
\end{aligned}
$$

where $\left(\mathbb{U}_{\epsilon}^{ \pm}, \Psi_{\epsilon}\right)$ satisfies the singular shock problem (here $\left.\nabla^{\epsilon}=\partial_{x^{\prime}}+\frac{\beta \partial_{\theta_{0}}}{\epsilon}\right)$ : 
(a) $\mathbb{P}^{ \pm}\left(\mathbb{U}_{\epsilon}^{ \pm}, \nabla^{\epsilon} \Psi_{\epsilon}\right) \partial_{x, \theta_{0}}^{\epsilon} \mathbb{U}_{\epsilon}^{ \pm}:=\partial_{x_{d}} \mathbb{U}_{\epsilon}^{ \pm}+\sum_{j=0}^{d-1} \tilde{A}_{j}\left(\mathbb{U}_{\epsilon}^{ \pm}, \nabla^{\epsilon} \Psi_{\epsilon}\right)\left(\partial_{x_{j}}+\frac{\beta_{j} \partial_{\theta_{0}}}{\epsilon}\right) \mathbb{U}_{\epsilon}^{ \pm}=0$

(b) $G\left(\mathbb{U}_{\epsilon}^{ \pm}, \nabla^{\epsilon} \Psi_{\epsilon}\right):=\sum_{j=0}^{d-1}\left[f_{j}\left(\mathbb{U}_{\epsilon}\right)\right]\left(\partial_{x_{j}}+\frac{\beta_{j} \partial_{\theta_{0}}}{\epsilon}\right) \Psi_{\epsilon}-\left[f_{d}\left(\mathbb{U}_{\epsilon}\right)\right]=0$ on $x_{d}=0$

(c) $\mathbb{U}_{\epsilon}^{ \pm}=\underline{u}^{ \pm}+\epsilon w_{\epsilon}^{ \pm}\left(x^{\prime \prime}, x_{d}, \theta_{0}\right)$ on $x_{0}=0$

(d) $\Psi_{\epsilon}=\sigma x_{0}+\chi_{\epsilon}\left(x^{\prime}, \theta_{0}\right)=0$ with $\chi_{\epsilon}=0$ on $x_{0}=0$,

where

$$
\tilde{A}_{j}\left(\mathbb{U}_{\epsilon}, \nabla^{\epsilon} \psi_{\epsilon}\right)=\mathcal{A}_{d}\left(\mathbb{U}_{\epsilon}, \nabla^{\epsilon} \psi_{\epsilon}\right)^{-1} A_{j}\left(\mathbb{U}_{\epsilon}\right), j=0, \ldots, d-1 .
$$

Corner compatibility conditions guarantee that smoothness only fails at the shock, and will also allow us to replace (3.27) by an equivalent forward problem (3.39) with data equal to zero in $x_{0}<0$. Phase compatibility conditions prevent the appearance of phases not in the allowed set $\left\{\phi_{m}^{ \pm}, m=1, \ldots, M^{ \pm}\right\}$.

A simple way to construct a phase-compatible $w_{\epsilon}^{+}\left(x^{\prime \prime}, x_{d}, \theta_{0}\right)$, for example, is to first choose a phase $\phi_{b}^{+}$from the set (3.16) that is outgoing from $x_{d}>0$ and use single-phase geometric optics [7] to construct an exact solution $v_{\epsilon}^{+}(x)$ to the pure initial value problem on $\mathbb{O}_{T_{0}}:=\left[-T_{0}, T_{0}\right] \times \mathbb{R}^{d}:$

$$
\begin{aligned}
& P^{+}\left(\underline{u}^{+}+\epsilon v_{\epsilon}^{+}, d_{x^{\prime}}\left(\sigma x_{0}\right)\right) \partial_{x} v_{\epsilon}^{+}=0 \text { on } \mathbb{O}_{T_{0}} \\
& v_{\epsilon}^{+}=\left.\nu^{+}\left(x^{\prime \prime}, x_{d}, \theta_{p}^{+}\right)\right|_{\theta_{p}^{+}=\frac{\phi_{p}^{+}}{\epsilon}} \text { on } x_{0}=0,
\end{aligned}
$$

where $\nu^{+}$is supported in $x_{d} \geq 0$ and vanishes to high order at $x_{d}=0$. Provided $\nu^{+}$ satisfies appropriate phase compatibility conditions, the construction of Gues [7], Theorem 1 , produces an exact solution of (3.29) of the form

$$
v_{\epsilon}^{+}(x)=\left.\left(\mathcal{W}^{0+}\left(x, \theta_{b}^{+}\right)+\epsilon \mathcal{W}_{\epsilon}^{1+}\left(x, \theta_{b}^{+}\right)\right)\right|_{\theta_{b}^{+}=\frac{\phi_{b}^{+}}{\epsilon}}+\epsilon^{2} \omega_{\epsilon}^{+}(x),
$$

where $\mathcal{W}^{0+}$ and $\mathcal{W}_{\epsilon}^{1+}$ are $C^{K}$ in $\left(x, \theta_{b}^{+}\right)$for $K$ large, periodic in $\theta_{b}^{+}$, and

$$
\left|\partial_{x}^{k} \omega_{\epsilon}^{+}(x)\right| \leq M, \text { for } k \leq K, \epsilon \in\left(0, \epsilon_{0}\right] .
$$

We now define

$$
\begin{aligned}
& W_{\epsilon}^{+}\left(x, \theta_{0}\right)=\mathcal{W}^{0+}\left(x, \theta_{0}+\frac{\underline{\omega}_{b}^{+} x_{d}}{\epsilon}\right)+\epsilon \mathcal{W}_{\epsilon}^{1+}\left(x, \theta_{0}+\frac{\underline{\omega}_{b}^{+} x_{d}}{\epsilon}\right)+\epsilon^{2} \omega_{\epsilon}^{+}(x), \\
& w_{\epsilon}^{+}\left(x^{\prime \prime}, x_{d}, \theta_{0}\right)=\left.W_{\epsilon}^{+}\left(x, \theta_{0}\right)\right|_{x_{0}=0} .
\end{aligned}
$$

Similarly, we construct $w_{\epsilon}^{-}\left(x^{\prime \prime}, x_{d}, \theta_{0}\right)$, where for example $\mathcal{W}^{0-}\left(x, \theta_{0}+\frac{\underline{\omega}_{a}^{-} x_{d}}{\epsilon}\right)$ now appears in the leading term of $W_{\epsilon}^{-}\left(x, \theta_{0}\right)$ and $\phi_{a}^{-}$is outgoing from $x_{d}<0 .{ }^{9}$

\footnotetext{
${ }^{9}$ The uniform stability condition implies that the total number of outgoing phases is $N+1$, so there is at least one outgoing phase for each choice of sign.
} 
Remark 3.5. Observe that $W_{\epsilon}^{ \pm}\left(x, \theta_{0}\right)$ satisfy the singular initial-value problems

$$
\begin{aligned}
& \text { (a) } \mathbb{P}^{ \pm}\left(\underline{u}^{ \pm}+\epsilon W_{\epsilon}^{ \pm}, \nabla^{\epsilon}\left(\sigma x_{0}\right)\right) \partial_{x, \theta_{0}}^{\epsilon}\left(\underline{u}^{ \pm}+W_{\epsilon}^{ \pm}\right)=0 \text { on } \mathbb{O}_{T_{0}} \\
& \text { (b) } W_{\epsilon}^{ \pm}=w_{\epsilon}^{ \pm}\left(x^{\prime \prime}, x_{d}, \theta_{0}\right) \text { on } x_{0}=0 .
\end{aligned}
$$

Corner compatibility for (3.27) is guaranteed by the high order vanishing of $w_{\epsilon}^{ \pm}$at $x_{d}=0$ and this, together with (3.33), insures for $k_{0}$ large that

$$
\begin{gathered}
\partial_{x_{0}}^{j} \mathcal{G}_{\epsilon}\left(x^{\prime}, \theta_{0}\right)=0 \text { at } x_{0}=0 \text { for } 0 \leq j \leq k_{0}, \text { where } \\
\epsilon \mathcal{G}_{\epsilon}\left(x^{\prime}, \theta_{0}\right):=G\left(\underline{u}^{ \pm}+\epsilon W_{\epsilon}^{ \pm}, \nabla^{\epsilon}\left(\sigma x_{0}\right)\right) .
\end{gathered}
$$

Letting

$$
\mathcal{G}_{\epsilon}\left(x^{\prime}, \theta_{0}\right)_{c}=\left\{\begin{array}{l}
\mathcal{G}_{\epsilon}, x_{0} \geq 0 \\
0, x_{0}<0
\end{array},\right.
$$

we see that by taking $K(3.31)$ and $k_{0}$ large enough we can guarantee that for any $s_{0}$,

(a) $\left\{\left(W_{\epsilon}^{ \pm}, \epsilon \partial_{x_{d}} W_{\epsilon}^{ \pm}\right): \epsilon \in\left(0, \epsilon_{0}\right]\right\}$ is bounded in $C\left(x_{d}, H^{s_{0}+1}\left(x^{\prime}, \theta_{0}\right)\right) \times C\left(x_{d}, H^{s_{0}}\left(x^{\prime}, \theta_{0}\right)\right)$

(b) $\left\{\left(\mathcal{G}_{\epsilon}\right)_{c}: \epsilon \in\left(0, \epsilon_{0}\right]\right\}$ is bounded in $H^{s_{0}+1}\left(x^{\prime}, \theta_{0}\right)$

We refer to Appendix A of [21] for an explanation of how to choose simultaneously cornerand phase-compatible data in the more general case where the initial data $w_{\epsilon}\left(x^{\prime \prime}, x_{d}, \theta_{0}\right)$ does not vanish at $x_{d}=0$.

If we now look for a solution to (3.27) of the form

$$
\begin{aligned}
& \mathbb{U}_{\epsilon}^{ \pm}\left(x, \theta_{0}\right)=\underline{u}^{ \pm}+\epsilon W_{\epsilon}^{ \pm}\left(x, \theta_{0}\right)+\epsilon U_{\epsilon}^{ \pm}\left(x, \theta_{0}\right) \\
& \Psi_{\epsilon}\left(x, \theta_{0}\right)=\sigma x_{0}+\epsilon \chi_{\epsilon}\left(x^{\prime}, \theta_{0}\right),
\end{aligned}
$$

we can use (3.33) to rewrite $(3.27)$ as a problem for $\left(U_{\epsilon}^{ \pm}, \chi_{\epsilon}\right)$ on $\left(-\infty, T_{0}\right] \times \mathbb{R}^{d}$ with data equal to zero in $x_{0} \leq 0$ :

$$
\begin{aligned}
& \text { (a) } \partial_{x_{d}} U_{\epsilon}^{ \pm}+\sum_{j=0}^{d-1} \tilde{A}_{j}\left(\underline{u}^{ \pm}+\epsilon W_{\epsilon}^{ \pm}+\epsilon U_{\epsilon}^{ \pm}, \nabla^{\epsilon}\left(\sigma x_{0}+\epsilon \chi_{\epsilon}\right)\right)\left(\partial_{x_{j}}+\frac{\beta_{j} \partial_{\theta_{0}}}{\epsilon}\right) U_{\epsilon}^{ \pm}= \\
& \quad-\sum_{j=0}^{d-1}\left(\tilde{A}_{j}\left(\underline{u}^{ \pm}+\epsilon W_{\epsilon}^{ \pm}+\epsilon U_{\epsilon}^{ \pm}, \nabla^{\epsilon}\left(\sigma x_{0}+\epsilon \chi_{\epsilon}\right)\right)-\tilde{A}_{j}\left(\underline{u}^{ \pm}+\epsilon W_{\epsilon}^{ \pm}, \nabla^{\epsilon}\left(\sigma x_{0}\right)\right)\right)\left(\partial_{x_{j}}+\frac{\beta_{j} \partial_{\theta_{0}}}{\epsilon}\right) W_{\epsilon}^{ \pm} \\
& \text {(b) } \sum_{j=0}^{d-1}\left(\int_{0}^{1}\left[f_{j}\left(\underline{u}+\epsilon W_{\epsilon}+s \epsilon U_{\epsilon}\right)\right] d s\right)\left(\partial_{x_{j}}+\frac{\beta_{j} \partial_{\theta_{0}}}{\epsilon}\right) \chi_{\epsilon}- \\
& \int_{0}^{1}\left[\mathcal{A}_{d}\left(\underline{u}+\epsilon W_{\epsilon}+s \epsilon U_{\epsilon}, \nabla^{\epsilon}\left(\sigma x_{0}+s \epsilon \chi_{\epsilon}\right)\right) U_{\epsilon}\right] d s=-\mathcal{G}_{\epsilon}\left(x^{\prime}, \theta_{0}\right)_{c} \\
& \text { (c) } U_{\epsilon}=0 \text { and } \chi_{\epsilon}=0 \text { in } x_{0}<0 .
\end{aligned}
$$


Below we say that

$$
U_{\epsilon}\left(x, \theta_{0}\right) \in E_{T}^{s} \Leftrightarrow U_{\epsilon}^{+} \in E_{T}^{s} \text { and } U_{\epsilon}^{-}\left(\cdot,-x_{d}, \cdot\right) \in E_{T}^{s}
$$

for $E_{T_{0}}^{s}$ as in Definition 2.1, and define

$$
\left|U_{\epsilon}\left(x, \theta_{0}\right)\right|_{E_{T}^{s}}=\left|U_{\epsilon}^{+}\left(x, \theta_{0}\right)\right|_{E_{T}^{s}}+\left|U_{\epsilon}^{-}\left(x, \theta_{0}\right)\right|_{E_{T}^{s}} .
$$

The spaces $\mathcal{E}_{T}^{s}$ and $\mathcal{P}_{T}^{s}$ and their norms are defined similarly in the shock context. The following existence theorem for (3.39) is proved in [23].

Theorem 3.6 ([23], Theorem 9.4). Let $s \geq\left[M_{0}+\frac{d+1}{2}\right]$ and set $s_{0}=s+1$. Consider the singular shock problem (3.39) and the associated nonsingular problem (3.25). Assume $W_{\epsilon}^{ \pm}$ is chosen as in (3.32) with compact $x$-support and so that (3.37) holds with $s_{0}=s+1$. There exist an $\epsilon_{0}>0, a T_{0}$ independent of $\epsilon \in\left(0, \epsilon_{0}\right]$, and a unique $\left(U_{\epsilon}, \chi_{\epsilon}\right) \in E_{T_{0}}^{s} \times H_{T_{0}}^{s+1}$ satisfying the singular problem (3.39) and such that for $\mathbb{U}_{\epsilon}^{ \pm}, \Psi_{\epsilon}\left(x^{\prime}, \theta_{0}\right)$ defined by (3.38)

$$
\begin{aligned}
& u_{\epsilon}^{ \pm}(x)=\left.\mathbb{U}_{\epsilon}^{ \pm}\left(x, \theta_{0}\right)\right|_{\theta_{0}=\frac{\beta x^{\prime}}{\epsilon}} \\
& \psi_{\epsilon}\left(x^{\prime}\right)=\left.\Psi_{\epsilon}\left(x^{\prime}, \theta_{0}\right)\right|_{\theta_{0}=\frac{\beta x^{\prime}}{\epsilon}},
\end{aligned}
$$

satisfies the original problem (3.25).

\subsection{Profile equations}

To derive the analogue of the almost-periodic profile equations (1.50) for shocks, we look for a corrected approximate solution of (3.25) of the form

$$
\begin{aligned}
& u_{\epsilon}^{c \pm}(x)=\left.\left(\underline{u}^{ \pm}+\epsilon \mathcal{W}^{0 \pm}\left(x, \theta_{0}, \xi_{d}\right)+\epsilon\left(\mathcal{U}^{0 \pm}\left(x, \theta_{0}, \xi_{d}\right)+\epsilon \mathcal{U}^{1 \pm}\left(x, \theta_{0}, \xi_{d}\right)\right)\right)\right|_{\theta_{0}=\frac{\phi_{0}}{\epsilon}, \xi_{d}=\frac{x_{d}}{\epsilon}} \\
& \psi_{\epsilon}^{c}\left(x^{\prime}\right)=\sigma x_{0}+\left.\epsilon\left(\chi^{0}\left(x^{\prime}, \theta_{0}\right)+\epsilon \chi^{1}\left(x^{\prime}, \theta_{0}\right)\right)\right|_{\theta_{0}=\frac{\phi_{0}}{\epsilon}},
\end{aligned}
$$

where with slight abuse we have set $\mathcal{W}^{0+}\left(x, \theta_{0}, \xi_{d}\right)=\mathcal{W}^{0+}\left(x, \theta_{0}+\underline{\omega}_{b}^{+} \xi_{d}\right)$ for $\mathcal{W}^{0+}\left(x, \theta_{b}^{+}\right)$as in $(3.30), \chi^{j}\left(x^{\prime}, \theta_{0}\right)$ are periodic in $\theta_{0}$, and $\mathcal{U}^{j \pm}$ have expansions like

$$
\mathcal{U}^{0 \pm}\left(x, \theta_{0}, \xi_{d}\right)=\sum_{\kappa=\left(\kappa_{0}, \kappa_{d}\right) \in \mathbb{Z} \times \mathbb{R}} U_{\kappa}^{ \pm}(x) e^{i \kappa_{0} \theta_{0}+i \kappa_{d} \xi_{d}} .
$$

In place of (1.35) we now define the sets of characteristic modes

$$
\mathcal{C}^{ \pm}=\left\{\kappa \in \mathbb{Z} \times \mathbb{R}: \operatorname{det} L^{ \pm}\left(\kappa_{0} \beta, \kappa_{d}\right)=0\right\},
$$

which can be decomposed as

$$
\mathcal{C}^{ \pm}=\cup_{m=1}^{M^{ \pm}} \mathcal{C}_{m}^{ \pm}, \text {where } \mathcal{C}_{m}^{ \pm}=\left\{\kappa_{0}\left(1, \underline{\omega}_{m}^{ \pm}\right): \kappa_{0} \in \mathbb{Z}\right\} .
$$


Setting

$$
\pi_{\kappa}^{ \pm}=\left\{\begin{array}{l}
P_{m}^{ \pm}, \text {if } \kappa \in \mathcal{C}_{m}^{ \pm} \backslash 0 \\
I, \text { if } \kappa=0 \\
0, \text { if } \kappa \notin \mathcal{C}^{ \pm}
\end{array}\right.
$$

we define the projectors

$$
\mathbb{E}^{ \pm} \mathcal{U}^{0 \pm}\left(x, \theta_{0}, \xi_{d}\right):=\sum_{\kappa=\left(\kappa_{0}, \kappa_{d}\right) \in \mathbb{Z} \times \mathbb{R}} \pi_{\kappa}^{ \pm} U_{\kappa}^{ \pm}(x) e^{i \kappa_{0} \theta_{0}+i \kappa_{d} \xi_{d}} .
$$

In writing the profile equations we use the following notation.

Notations 3.7.

1. $\tilde{L}^{ \pm}\left(\partial_{x}\right)=\partial_{x_{d}}+\sum_{j=0}^{d-1} \tilde{A}_{j}\left(\underline{u}^{ \pm}, d_{x^{\prime}}\left(\sigma x_{0}\right)\right) \partial_{x_{j}}$.

2. $\tilde{\mathcal{L}}^{ \pm}\left(\partial_{\theta_{0}}, \partial_{\xi_{d}}\right)=\tilde{L}^{ \pm}\left(d \phi_{0}\right) \partial_{\theta_{0}}+\tilde{L}^{ \pm}\left(d x_{d}\right) \partial_{\xi_{d}}=\partial_{\xi_{d}}+\sum_{j=0}^{d-1} \tilde{A}_{j}\left(\underline{u}^{ \pm}, d_{x^{\prime}}\left(\sigma x_{0}\right)\right) \beta_{j} \partial_{\theta_{0}}$.

3. $\mathcal{M}_{1}^{ \pm}\left(\mathcal{U}^{0 \pm}\right) \partial_{\theta_{0}}=\left(\sum_{j=0}^{d-1} \partial_{u} \tilde{A}_{j}\left(\underline{u}^{ \pm}, d_{x^{\prime}}\left(\sigma x_{0}\right)\right) \cdot \mathcal{U}^{0 \pm} \beta_{j}\right) \partial_{\theta_{0}}$.

4. With $\tilde{A}_{j}\left(\underline{u}^{ \pm}, d_{x^{\prime}} \psi\right)=\left.\tilde{A}_{j}\left(\underline{u}^{ \pm}, \omega\right)\right|_{\omega=d_{x^{\prime}}} \psi$ define

$$
\mathcal{M}_{2}^{ \pm}\left(d_{x^{\prime}} \chi^{0}, \partial_{\theta_{0}} \chi^{1}\right) \partial_{\theta_{0}}=\left(\sum_{j=0}^{d-1} \partial_{\omega} \tilde{A}_{j}\left(\underline{u}^{ \pm}, d_{x^{\prime}}\left(\sigma x_{0}\right)\right) \cdot\left(d_{x^{\prime}} \chi^{0}+\beta \partial_{\theta_{0}} \chi^{1}\right) \beta_{j}\right) \partial_{\theta_{0}} \quad(\text { see }(3.50)) \text {. }
$$

The almost-periodic profile equations for the unknowns $\mathcal{U}^{0 \pm}\left(x, \theta_{0}, \xi_{d}\right), \chi^{0}\left(x^{\prime}, \theta_{0}\right)$, and $\chi^{1}\left(x^{\prime}, \theta_{0}\right)$ are derived by the procedure used in section 2.1. We write them here suppressing all superscripts \pm :

(a) $\mathbb{E} \mathcal{U}^{0}=\mathcal{U}^{0}$

(b) $\mathbb{E}\left(\tilde{L}\left(\partial_{x}\right) \mathcal{U}^{0}+\mathcal{M}_{1}\left(\mathcal{W}^{0}+\mathcal{U}^{0}\right) \partial_{\theta_{0}} \mathcal{U}^{0}+\mathcal{M}_{2}\left(d_{x^{\prime}} \chi^{0}, \partial_{\theta_{0}} \chi^{1}\right) \partial_{\theta_{0}} \mathcal{U}^{0}\right)=$ $-\mathbb{E}\left(\mathcal{M}_{1}\left(\mathcal{U}^{0}\right) \partial_{\theta_{0}} \mathcal{W}^{0}+\mathcal{M}_{2}\left(d_{x^{\prime}} \chi^{0}, \partial_{\theta_{0}} \chi^{1}\right) \partial_{\theta_{0}} \mathcal{W}^{0}\right)$

(c) $G(\beta) \partial_{\theta_{0}} \chi^{0}:=\sum_{j=0}^{d-1}\left[f_{j}(\underline{u})\right] \beta_{j} \partial_{\theta_{0}} \chi^{0}=0$

(d) $G(\beta) \partial_{\theta_{0}} \chi^{1}+\sum_{j=0}^{d-1}\left[f_{j}(\underline{u})\right] \partial_{x_{j}} \chi^{0}+\left[\left(\sigma A_{0}(\underline{u})-A_{d}(\underline{u})\right) \mathcal{U}^{0}\right]=\left[\left(A_{d}(\underline{u})-\sigma A_{0}(\underline{u})\right) \mathcal{W}^{0}\right]_{c}$

(e) $\mathcal{U}^{0}=0, \chi^{0}=0$, and $\chi^{1}=0$ in $x_{0} \leq 0$.

Observe that uniform stability and (3.49)(c) imply

$$
\partial_{\theta_{0}} \chi^{0}=0
$$


so henceforth we write $\chi^{0}=\chi^{0}\left(x^{\prime}\right)$. We also have

$$
\tilde{\mathcal{L}}\left(\partial_{\theta_{0}}, \partial_{\xi_{d}}\right) \mathcal{U}^{0}=0 \Leftrightarrow \mathbb{E} \mathcal{U}^{0}=\mathcal{U}^{0} .
$$

In deriving (3.49) we have used the fact that

$$
\begin{aligned}
& \mathbb{E} \mathcal{W}^{0}=\mathcal{W}^{0} \\
& \mathbb{E}\left(\tilde{L}\left(\partial_{x}\right) \mathcal{W}^{0}+\mathcal{M}_{1}\left(\mathcal{W}^{0}\right) \partial_{\theta_{0}} \mathcal{W}^{0}\right)=0,
\end{aligned}
$$

a consequence of (3.29),(3.30) or, alternatively, the construction of [7].

In order to write down the equivalent periodic profile equations we must treat the phase $\phi_{0}$ on an equal footing with the phases $\phi_{m}^{ \pm}$(3.16). It is possible that $\phi_{0}$ already belongs to one or both of the sets $S^{ \pm}=\left\{\phi_{m}^{ \pm}, m=1, \ldots, M^{ \pm}\right\}$. We shall consider the generic case where $\phi_{0}$ does not belong to either of the sets $S^{ \pm}$. The other cases require only minor changes.

Recall the notation $\mathbb{Z}^{M ; k}, H^{s ; k}\left(\overline{\mathbb{R}}_{+}^{d+1} \times \mathbb{T}^{M}\right)$ introduced in (2.11), (2.12).

Definition 3.8. Let

$$
\theta^{ \pm}=\left(\theta_{0}, \theta_{1}^{ \pm}, \ldots, \theta_{M^{ \pm}}^{ \pm}\right) \in \mathbb{T}^{M^{ \pm}+1}
$$

be a placeholder for $\frac{1}{\epsilon} \phi^{ \pm}:=\frac{1}{\epsilon}\left(\phi_{0}, \phi_{1}^{ \pm}, \ldots, \phi_{M^{ \pm}}^{ \pm}\right)$. We say $\alpha^{ \pm} \in \mathbb{Z}^{M^{ \pm}+1 ; 2}$ is a characteristic mode and write $\alpha^{ \pm} \in \mathcal{C}^{ \pm}$if $\operatorname{det} L^{ \pm}\left(d\left(\alpha^{ \pm} \cdot \phi^{ \pm}\right)\right)=0$. We decompose

$$
\begin{aligned}
& \mathcal{C}^{\prime \pm}=\cup_{m=1}^{M_{ \pm}} \mathcal{C}_{m}^{\prime \pm} \\
& \text { where } \mathcal{C}_{m}^{\prime \pm}=\left\{\alpha^{ \pm} \in \mathbb{Z}^{M^{ \pm}+1 ; 2}: \alpha^{ \pm} \cdot \phi^{ \pm}=n_{\alpha^{ \pm}} \phi_{m}^{ \pm} \text {for some } n_{\alpha^{ \pm}} \in \mathbb{Z}\right\} .
\end{aligned}
$$

For $\mathcal{V}\left(x, \theta^{+}\right)=\sum_{\alpha \in \mathbb{Z}^{M^{+}+1 ; 2}} V_{\alpha}(x) e^{i \alpha \cdot \theta^{+}} \in H^{s ; 2}\left(\overline{\mathbb{R}}_{+}^{d+1} \times \mathbb{T}^{M^{+}+1}\right)$ define the projector

$$
\mathbb{E}^{\prime+}=\mathbb{E}_{0}^{\prime}+\sum_{m=1}^{M^{+}} \mathbb{E}_{m}^{+}, \text {where } \mathbb{E}_{0}^{\prime} \mathcal{V}=V_{0} \text { and } \mathbb{E}_{m}^{\prime+} \mathcal{V}=\sum_{\alpha \in \mathcal{C}_{m}^{\prime+} \backslash 0} P_{m}^{+} V_{\alpha}(x) e^{i n_{\alpha} \theta_{m}^{+}}
$$

The projector $\mathbb{E}^{\prime}$ is defined similarly on periodic functions $\mathcal{V}\left(x, \theta^{-}\right)$. Setting $\mathcal{L}^{\prime}\left(\partial_{\theta^{+}}\right)=$ $\sum_{m=0}^{M^{+}} \tilde{L}^{+}\left(d \phi_{m}^{+}\right) \partial_{\theta_{m}^{+}}\left(\right.$here $\left.\phi_{0}^{+}=\phi_{0}\right)$, we note that

$$
\mathbb{E}^{\prime}+\mathcal{V}=\mathcal{V} \text { if and only if } \mathcal{V} \in H^{s ; 1}\left(\overline{\mathbb{R}}_{+}^{d+1} \times \mathbb{T}^{M^{+}+1}\right) \text { and } \mathcal{L}^{\prime+}\left(\partial_{\theta^{+}}\right) \mathcal{V}=0
$$

a condition that is equivalent to the property that $\mathcal{V}\left(x, \theta^{+}\right)$has an expansion of the form

$$
\mathcal{V}=\underline{v}(x)+\sum_{m=1}^{M^{+}} \sum_{k=1}^{\nu_{k_{m}}^{+}} \sigma_{m, k}\left(x, \theta_{m}^{+}\right) r_{m, k}^{+}:=\underline{v}(x)+\sum_{m=1}^{M^{+}} \mathcal{V}_{m}\left(x, \theta_{m}^{+}\right) \text {. }
$$


We let

$$
\begin{aligned}
& \mathcal{M}_{1}^{\prime \pm}\left(\mathcal{V}^{0 \pm}\right) \partial_{\theta^{ \pm}}=\sum_{m=0}^{M^{ \pm}}\left(\sum_{j=0}^{d-1} \partial_{u} \tilde{A}_{j}\left(\underline{u}^{ \pm}, d\left(\sigma x_{0}\right)\right) \cdot \mathcal{V}^{0 \pm} \beta_{j}\right) \partial_{\theta_{m}^{ \pm}} \\
& \mathcal{M}_{2}^{\prime \pm}\left(d_{x^{\prime}} \chi^{0}, \partial_{\theta_{0}} \chi^{1}\right) \partial_{\theta^{ \pm}}=\sum_{m=0}^{M^{ \pm}}\left(\sum_{j=0}^{d-1} \partial_{\omega} \tilde{A}_{j}\left(\underline{u}^{ \pm}, d\left(\sigma x_{0}\right)\right) \cdot\left(d_{x^{\prime}} \chi^{0}+\beta \partial_{\theta_{0}} \chi^{1}\right) \beta_{j}\right) \partial_{\theta_{m}^{ \pm}},
\end{aligned}
$$

and with abuse we define $\mathcal{W}^{0 \pm}\left(x, \theta^{ \pm}\right)$by

$$
\mathcal{W}^{0+}\left(x, \theta^{+}\right)=\mathcal{W}^{0+}\left(x, \theta_{b}^{+}\right) ; \mathcal{W}^{0-}\left(x, \theta^{-}\right)=\mathcal{W}^{0-}\left(x, \theta_{a}^{+}\right) \quad(\operatorname{recall}(3.32)) .
$$

Suppressing all superscripts \pm , we can now write the periodic profile equations for the unknowns $\mathcal{V}^{0}(x, \theta), \chi^{0}\left(x^{\prime}, \theta_{0}\right)$, and $\chi^{1}\left(x^{\prime}, \theta_{0}\right)$ :

(a) $\mathbb{E}^{\prime} \mathcal{V}^{0}=\mathcal{V}^{0}$

(b) $\mathbb{E}^{\prime}\left(\tilde{L}\left(\partial_{x}\right) \mathcal{V}^{0}+\mathcal{M}_{1}^{\prime}\left(\mathcal{W}^{0}+\mathcal{V}^{0}\right) \partial_{\theta} \mathcal{V}^{0}+\mathcal{M}_{2}^{\prime}\left(d_{x^{\prime}} \chi^{0}, \partial_{\theta_{0}} \chi^{1}\right) \partial_{\theta} \mathcal{V}^{0}\right)=$ $-\mathbb{E}^{\prime}\left(\mathcal{M}_{1}^{\prime}\left(\mathcal{V}^{0}\right) \partial_{\theta} \mathcal{W}^{0}+\mathcal{M}_{2}^{\prime}\left(d_{x^{\prime}} \chi^{0}, \partial_{\theta_{0}} \chi^{1}\right) \partial_{\theta} \mathcal{W}^{0}\right)$

(c) $G(\beta) \partial_{\theta_{0}} \chi^{0}:=\sum_{j=0}^{d-1}\left[f_{j}(\underline{u})\right] \beta_{j} \partial_{\theta_{0}} \chi^{0}=0$

(d) $G(\beta) \partial_{\theta_{0}} \chi^{1}+\sum_{j=0}^{d-1}\left[f_{j}(\underline{u})\right] \partial_{x_{j}} \chi^{0}+\left[\left(\sigma A_{0}(\underline{u})-A_{d}(\underline{u})\right) \mathcal{V}^{0}\right]=\left[\left(A_{d}(\underline{u})-\sigma A_{0}(\underline{u})\right) \mathcal{W}^{0}\right]_{c}$

(e) $\mathcal{V}^{0}=0, \chi^{0}=0$, and $\chi^{1}=0$ in $x_{0} \leq 0$.

The terms involving $\mathcal{M}_{1}^{\prime}$ describe interior-interior interactions, some of which now involve the wave $\mathcal{W}^{0}$ which generates the perturbation. The terms involving $\mathcal{M}_{2}^{\prime}$ describe shock surface-interior interactions.

As in the fixed boundary case we will construct $\left(\mathcal{U}^{0}, \chi^{0}, \chi^{1}\right)$ satisfying $(3.49)$ by constructing $\left(\mathcal{V}^{0}, \chi^{0}, \chi^{1}\right)$ satisfying $(3.60)$ and then setting

$$
\mathcal{U}^{0 \pm}\left(x, \theta_{0}, \xi_{d}\right)=\mathcal{V}^{0 \pm}\left(\theta_{0}, \theta_{0}+\underline{\omega}_{1}^{ \pm} \xi_{d}, \ldots, \theta_{0}+\underline{\omega}_{M^{ \pm}}^{ \pm} \xi_{d}\right) .
$$

Moreover, with abuse we define

$$
\mathcal{W}^{0+}\left(x, \theta_{0}, \xi_{d}\right):=\mathcal{W}^{0+}\left(x, \theta_{0}+\underline{\omega}_{b} \xi_{d}\right), \mathcal{W}^{0-}\left(x, \theta_{0}, \xi_{d}\right):=\mathcal{W}^{0-}\left(x, \theta_{0}+\underline{\omega}_{a} \xi_{d}\right)
$$

for $\mathcal{W}^{0 \pm}$ as in (3.32).

The large system in the shock case. We shall write out the large system for the unknowns $\chi^{0}\left(x^{\prime}\right), \chi^{1}\left(x^{\prime}, \theta_{0}\right)$ and the terms $\underline{v}^{+}(x), \sigma_{m, k}^{+}\left(x, \theta_{m}^{+}\right)$appearing in the expansion

$$
\mathcal{V}^{0+}\left(x, \theta^{+}\right)=\underline{v}^{+}(x)+\sum_{m=0}^{M^{+}} \sum_{k=1}^{\nu_{k_{m}}^{+}} \sigma_{m, k}^{+}\left(x, \theta_{m}^{+}\right) r_{m, k}^{+} .
$$


Even though $\mathcal{W}^{0+}$ depends just on the single phase $\phi_{b}^{+}$, we can avoid enumerating some cases by denoting $\mathcal{W}^{0+}$ similarly:

$$
\mathcal{W}^{0+}\left(x, \theta^{+}\right)=\underline{w}^{+}(x)+\sum_{m=0}^{M^{+}} \sum_{k=1}^{\nu_{k_{m}}^{+}} \tilde{\sigma}_{m, k}^{+}\left(x, \theta_{m}^{+}\right) r_{m, k}^{+}, \text {where } \tilde{\sigma}_{m, k}^{+}=0 \text { unless } m=b .
$$

We shall consider an illustrative situation where there are precisely two ordered triples of resonant phases among the $\phi_{m}^{+}, m=0, \ldots, M^{+}$:

$$
\begin{aligned}
& \text { (a) } n_{q}^{+} \phi_{q}^{+}=n_{p}^{+} \phi_{p}^{+}+n_{r}^{+} \phi_{r}^{+} \\
& \text {(b) } n_{0}^{+} \phi_{0}=\underline{n}_{p}^{+} \phi_{p}^{+}+n_{s}^{+} \phi_{s}^{+} \text {. }
\end{aligned}
$$

In addition to the prepared integrals $I_{n_{q}, n_{p}, n_{r}}^{k, k^{\prime}}\left(x, \theta_{p}\right)$ describing $\mathcal{V}^{0+} / \mathcal{V}^{0+}$ interactions defined previously (2.58), we now need similar integrals describing interactions of $\mathcal{W}^{0+}$ with $\mathcal{V}^{0+}$ (suppress ${ }^{+}$and ignore superscripts $n, n+1$ for now):

$$
\begin{aligned}
& \tilde{I}_{n_{q}, n_{p}, n_{r}}^{k, k^{\prime}}\left(x, \theta_{p}\right)=\frac{1}{2 \pi} \int_{0}^{2 \pi}\left(\tilde{\sigma}_{q, k}\right)_{n_{q}}\left(x, \frac{n_{p}}{n_{q}} \theta_{p}+\frac{n_{r}}{n_{q}} \theta_{r}\right) \partial_{\theta_{r}} \sigma_{r, k^{\prime}}^{n+1}\left(x, \theta_{r}\right) d \theta_{r}, \\
& \bar{I}_{n_{q}, k_{p}, n_{r}}^{k, n^{\prime}}\left(x, \theta_{p}\right)=\frac{1}{2 \pi} \int_{0}^{2 \pi}\left(\sigma_{q, k}^{n}\right)_{n_{q}}\left(x, \frac{n_{p}}{n_{q}} \theta_{p}+\frac{n_{r}}{n_{q}} \theta_{r}\right) \partial_{\theta_{r}} \tilde{\sigma}_{r, k^{\prime}}\left(x, \theta_{r}\right) d \theta_{r} .
\end{aligned}
$$

We also need new integrals describing shock-interior interactions:

$$
\begin{aligned}
& S_{n_{0}, \underline{n}_{p}, n_{s}}^{k}\left(x, \theta_{p}\right)=\frac{1}{2 \pi} \int_{0}^{2 \pi}\left(\partial_{\theta_{0}} \chi^{1, n}\right)_{n_{0}}\left(x^{\prime}, \frac{\underline{n}_{p}}{n_{0}} \theta_{p}+\frac{n_{s}}{n_{0}} \theta_{s}\right) \partial_{\theta_{s}} \sigma_{s, k}^{n+1}\left(x, \theta_{s}\right) d \theta_{s} \\
& \tilde{S}_{n_{0}, \underline{n}_{p}, n_{s}}^{k}\left(x, \theta_{p}\right)=\frac{1}{2 \pi} \int_{0}^{2 \pi}\left(\partial_{\theta_{0}} \chi^{1, n}\right)_{n_{0}}\left(x^{\prime}, \frac{\underline{n}_{p}}{n_{0}} \theta_{p}+\frac{n_{s}}{n_{0}} \theta_{s}\right) \partial_{\theta_{s}} \tilde{\sigma}_{s, k}\left(x, \theta_{s}\right) d \theta_{s}
\end{aligned}
$$

Applying the projectors $\mathbb{E}_{0}^{\prime}, \mathbb{E}_{p, l}^{\prime+}$ to $(3.49)(\mathrm{c})$, we obtain (suppress ${ }^{+}$):

$$
\begin{gathered}
\tilde{L}\left(\partial_{x}\right) \underline{v}^{n+1}+\sum_{j, m, k, k^{\prime}} \frac{1}{2 \pi}\left(\int_{0}^{2 \pi}\left(\sigma_{m, k}^{n}+\tilde{\sigma}_{m, k}\right)\left(x, \theta_{m}\right) \partial_{\theta_{m}} \sigma_{m, k^{\prime}}^{n+1}\left(x, \theta_{m}\right) d \theta_{m}\right) R_{j, m}^{k, k^{\prime}}= \\
-\sum_{j, m, k, k^{\prime}} \frac{1}{2 \pi}\left(\int_{0}^{2 \pi} \sigma_{m, k}^{n}\left(x, \theta_{m}\right) \partial_{\theta_{m}} \tilde{\sigma}_{m, k^{\prime}}\left(x, \theta_{m}\right) d \theta_{m}\right) R_{j, m}^{k, k^{\prime}} .
\end{gathered}
$$


(a) $X_{\phi_{p}} \sigma_{p, l}^{n+1}\left(x, \theta_{p}\right)+\sum_{j, k^{\prime}} a_{p, l, j}^{k^{\prime}}\left(\underline{w}+\underline{v}^{n}\right) \partial_{\theta_{p}} \sigma_{p, k^{\prime}}^{n+1}\left(x, \theta_{p}\right)+$

(b) $\sum_{j, k, k^{\prime}} b_{p, l, j}^{k, k^{\prime}}\left(\tilde{\sigma}_{p, k}+\sigma_{p, k}^{n}\right)\left(x, \theta_{p}\right) \partial_{\theta_{p}} \sigma_{p, k^{\prime}}^{n+1}\left(x, \theta_{p}\right)-\frac{1}{2 \pi} \int_{0}^{2 \pi}$ (previous sum) $d \theta_{p}+$

(c) $\sum_{j, k, k^{\prime}} c_{p, l, j}^{k, k^{\prime}}\left(\tilde{I}_{n_{q}, n_{p}, n_{r}}^{k, k^{\prime}}+I_{n_{q}, n_{p}, n_{r}}^{k, k^{\prime}}\right)\left(x, \theta_{p}\right)+\sum_{j, k, k^{\prime}} d_{p, l, j}^{k, k^{\prime}}\left(\tilde{I}_{n_{r},-n_{p}, n_{q}}^{k, k^{\prime}}+I_{n_{r},-n_{p}, n_{q}}^{k, k^{\prime}}\right)\left(x, \theta_{p}\right)+$

(d) $\sum_{j, k^{\prime}} e_{p, l, j}^{k^{\prime}}\left(\partial_{x^{\prime}} \chi^{0, n}\left(x^{\prime}\right)\right) \sigma_{p, k^{\prime}}^{n}\left(x, \theta_{p}\right)+\sum_{j, k} f_{p, l, j}^{k} S_{n_{0}, \underline{n}_{p}, n_{s}}^{k}\left(x, \theta_{p}\right)=$

(e) $-\left(\sum_{j, k, k^{\prime}} b_{p, l, j}^{k, k^{\prime}} \sigma_{p, k}^{n}\left(x, \theta_{p}\right) \partial_{\theta_{p}} \tilde{\sigma}_{p, k^{\prime}}\left(x, \theta_{p}\right)-\frac{1}{2 \pi} \int_{0}^{2 \pi}\right.$ (previous sum) $\left.d \theta_{p}\right)$

$(f)-\left(\sum_{j, k, k^{\prime}} c_{p, l, j}^{k, k^{\prime}} \bar{I}_{n_{q}, n_{p}, n_{r}}^{k, k^{\prime}}\left(x, \theta_{p}\right)+\sum_{j, k, k^{\prime}} d_{p, l, j}^{k, k^{\prime}} \bar{I}_{n_{r},-n_{p}, n_{q}}^{k, n^{\prime}}\left(x, \theta_{p}\right)\right)$

$(g)-\left(\sum_{j, k^{\prime}} e_{p, l, j}^{k^{\prime}}\left(\partial_{x^{\prime}} \chi^{0, n}\left(x^{\prime}\right)\right) \tilde{\sigma}_{p, k^{\prime}}\left(x, \theta_{p}\right)+\sum_{j, k} f_{p, l, j}^{k} \tilde{S}_{n_{0}, \underline{n}_{p}, n_{s}}^{k}\left(x, \theta_{p}\right)\right)$.

Remark 3.9. In cases where more triples of resonant phases than those in (3.65) are present on the plus side, additional prepared integrals similar to those in lines (3.69)(c),(f) appear for each triple that does not include $\phi_{0}$, while additional integrals like those in $(3.69)(\mathrm{d}),(\mathrm{g})$ appear for each triple that includes $\phi_{0}$. The formulas for the vectors $R_{j, m}^{k, k^{\prime}}$ and the scalars $a_{p, l, j}^{k^{\prime}}(\underline{w}+\underline{v}), b_{p, l, j}^{k, k^{\prime}}$, etc., appearing above are quite similar to those in the fixed boundary case. There is a similar collection of equations involving the unknowns $\chi^{0}, \chi^{1}$, and $\underline{v}^{-}$, $\sigma_{m, k}^{-}$on the - side, quite possibly with a different number (which can be zero) of prepared integrals.

The interior equations on the plus and minus sides are coupled by the following transmission conditions on $x_{d}=0$, which we separate into parts with nonzero mean and zero mean:

$$
\begin{aligned}
& \text { (a) } \sum_{j=0}^{d-1}\left[f_{j}(\underline{u})\right] \partial_{x_{j}} \chi^{0, n+1}+\left[\left(\sigma A_{0}(\underline{u})-A_{d}(\underline{u})\right) \underline{v}^{n+1}\right]=\left[\left(A_{d}(\underline{u})-\sigma A_{0}(\underline{u})\right) \underline{w}\right]_{c} \\
& \text { (b) } G(\beta) \partial_{\theta_{0}} \chi^{1 *, n+1}+\left[\left(\sigma A_{0}(\underline{u})-A_{d}(\underline{u})\right) \mathcal{V}^{0 *, n+1}\right]=\left[\left(A_{d}(\underline{u})-\sigma A_{0}(\underline{u})\right) \mathcal{W}^{0 *}\right]_{c} .
\end{aligned}
$$

Parallel to the fixed boundary case, uniform stability implies that (3.70)(b) can be rewritten as

$$
\begin{aligned}
& \left(\partial_{\theta_{0}} \chi^{1 *, n+1}, \sigma_{m, k}^{ \pm, n+1}\left(x^{\prime}, 0, \theta_{0}\right) ; m \in \mathbb{I}, k \in\left\{1, \ldots, \nu_{k_{m}}^{ \pm}\right\}\right)= \\
& \mathbb{B}\left(\mathcal{W}^{0 *}, \sigma_{m, k}^{ \pm, n+1}\left(x^{\prime}, 0, \theta_{0}\right) ; m \in \mathbb{O}, k \in\left\{1, \ldots, \nu_{k_{m}}^{ \pm}\right\}\right)
\end{aligned}
$$


where $\mathbb{B}$ is a well-determined linear function of its arguments.

In stating the main estimate for the profile iteration scheme, we use the same notation for spaces and norms introduced in (2.80), except that now $\theta^{ \pm} \in \mathbb{T}^{M^{ \pm}+1}$ and we write

$$
\mathcal{V}(x, \theta) \in \mathbb{H}_{T}^{m} \Leftrightarrow \mathcal{V}^{+} \in H^{m}\left(\Omega_{T, \theta^{+}}\right) \text {and } \mathcal{V}^{0-}\left(\cdot,-x_{d}, \cdot\right) \in H^{m}\left(\Omega_{T, \theta^{-}}\right) .
$$

Proposition 3.10. Let $T>0$ and $m>\frac{d+3}{2}+1$. Suppose that $\left(\mathcal{V}^{0, n}, \chi^{0, n}, \chi^{1, n}\right)$ vanishes in $t \leq 0$ with

$$
\left(\mathcal{V}^{0, n}, \chi^{0, n}\right) \in \mathbb{H}_{T}^{m} \times H_{T}^{m+1}, \quad \partial_{\theta_{0}} \chi^{1, n} \in H_{T}^{m},
$$

and suppose that $\mathcal{W}^{0} \in \mathbb{H}_{T}^{m+1}$ has compact $x$-support with

$$
\mathcal{H}\left(x^{\prime}, \theta_{0}\right):=\left.\left[\left(A_{d}(\underline{u})-\sigma A_{0}(\underline{u})\right) \mathcal{W}^{0}\right]_{c}\right|_{x_{d}=0} \in H_{T}^{m} .
$$

The coupled system (3.68),(3.69),(3.70) has a unique solution $\left(\mathcal{V}^{0, n+1}, \chi^{0, n+1}, \chi^{1, n+1}\right)$ vanishing in $t \leq 0$, with the same regularity as in (3.73), and such that $\chi^{1, n+1}\left(x, \theta_{0}\right)$ has mean zero. Moreover, there exist increasing functions, $\gamma_{0}(K)$ and $C(K)$, of

$$
K=\left|\mathcal{V}^{0, n}\right|_{m, T}+\left\langle d_{x^{\prime}} \chi^{0, n}, \partial_{\theta_{0}} \chi^{1, n}\right\rangle_{m, T}
$$

such that for $\gamma>\gamma_{0}(K)$ we have

$$
\begin{aligned}
& \left|\mathcal{V}^{0, n+1}\right|_{m, \gamma, T}+\frac{\left\langle\mathcal{V}^{0, n+1}, d_{x^{\prime}} \chi^{0, n+1}, \partial_{\theta_{0}} \chi^{1, n+1}\right\rangle_{m, \gamma, T}}{\sqrt{\gamma}} \leq \\
& C(K)\left(\frac{\left|\mathcal{V}^{0, n}\right|_{m, \gamma, T}+\left\langle d_{x^{\prime}} \chi^{0, n}, \partial_{\theta_{0}} \chi^{1, n}\right\rangle_{m, \gamma, T}}{\gamma}+\frac{\langle\mathcal{H}\rangle_{m, \gamma, T}}{\sqrt{\gamma}}\right) .
\end{aligned}
$$

Proof. The proof is very close to that of Proposition 2.19, but we will point out the new features.

1. The analogue of the critical Proposition 2.18 continues to hold as stated for the constants $b_{p, l, j}^{k, k^{\prime}}$ appearing in $(3.69)(\mathrm{b})$, and this allows us in the proof of the $L^{2}$ estimate to treat lines (3.69)(a) and (b) just as in Proposition 2.19. In the proof of Proposition 2.18 the matrix $\mathcal{A}(u, \beta)$ should now be replaced by

$$
\mathcal{A}^{ \pm}\left(u^{ \pm}, \beta\right):=-i \mathcal{A}_{d}^{-1}\left(u^{ \pm}, d_{x^{\prime}}\left(\sigma x_{0}\right)\right)\left(\left(\underline{\tau} A_{0}\left(u^{ \pm}\right)+\sum_{j=1}^{d-1} \underline{\eta}_{j} A_{j}\left(u^{ \pm}\right)\right) .\right.
$$

2. Again we obtain $L^{2}$ estimates for the problem where forcing terms $\underline{F}$ and $F^{*}$ are 
added to the right sides of (3.68) and (3.69) respectively. In place of (2.87) we have now

$$
\begin{aligned}
& \text { (a) }\left|\underline{v}^{n+1}\right|_{0, \gamma, T}+\frac{\left\langle\underline{v}^{n+1}, d_{x^{\prime}} \chi^{0, n+1}\right\rangle_{0, \gamma, T}}{\sqrt{\gamma}} \leq \\
& C\left(K^{\prime}\right)\left(\frac{\left|\left(\mathcal{V}^{0, *}\right)^{n+1}\right|_{0, \gamma, T}}{\gamma}+\frac{|\underline{F}|_{0, \gamma, T}}{\gamma}+\frac{\left|\left(\mathcal{V}^{0, *}\right)^{n}\right|_{0, \gamma, T}}{\gamma}+\frac{\langle\underline{\mathcal{H}}\rangle_{0, \gamma, T}}{\sqrt{\gamma}}\right) \\
& \left(\text { b) }\left|\left(\mathcal{V}^{0, *}\right)^{n+1}\right|_{0, \gamma, T}+\frac{\left\langle\left(\mathcal{V}^{0, *}\right)^{n+1}, \partial_{\theta_{0}} \chi^{1, n+1}\right\rangle_{0, \gamma, T}}{\sqrt{\gamma}} \leq\right. \\
& C\left(K^{\prime}\right)\left(\frac{\left|F^{*}\right|_{0, \gamma, T}}{\gamma}+\frac{\left|\left(\mathcal{V}^{0, *}\right)^{n}\right|_{0, \gamma, T}+\left\langle d_{x^{\prime}} \chi^{0, n}, \partial_{\theta_{0}} \chi^{1, n}\right\rangle_{0, \gamma, T}}{\gamma}+\frac{\left\langle\mathcal{H}^{*}\right\rangle_{0, \gamma, T}}{\sqrt{\gamma}}\right),
\end{aligned}
$$

where (3.78)(a) follows from the uniform stability of $\left(\tilde{L}(\partial), B\left(\underline{u}^{ \pm}, d_{x^{\prime}}\left(\sigma x_{0}\right)\right)\right.$ by applying the Majda [13] estimate to (3.68), (3.70)(a). In proving (3.78)(b), as before an integration by parts should first be done in integrals in which a $\theta$-derivative falls on an $n+1$-st iterate. The estimate of $\partial_{\theta_{0}} \chi^{1, n+1}$ in (3.78)(b) follows from the estimate for outgoing modes using (3.71). The terms involving $\chi^{0, n}, \chi^{1, n}$ on the right of (3.78)(b) arise from line (g) of (3.69). The estimate (3.76) for $m=0$ (with forcing $F$ ) follows from (3.78).

3. Higher tangential derivative estimates are proved by applying the $L^{2}$ estimate to the problem satisfied by $\gamma^{m-|\alpha|} \partial_{x^{\prime}, \theta}^{\alpha}\left(\mathcal{V}^{0, n+1}, d_{x^{\prime}} \chi^{0, n+1}, \partial_{\theta_{0}} \chi^{1, n+1}\right)$ and again depend on observations $\mathbf{A}$ and $\mathbf{B}$ in the proof of Proposition 2.19. For example, differentiating the prepared integral in $(3.69)(\mathrm{d})$ leads to new forcing terms

$$
\gamma^{m-|\alpha|} \int_{0}^{2 \pi}\left|\partial_{\left(x^{\prime}, \theta_{p}\right)}^{\alpha_{1}}\left(\partial_{\theta_{0}} \chi^{1, n}\right)_{n_{0}}\left(x^{\prime}, \frac{\underline{n}}{n_{0}} \theta_{p}+\frac{n_{s}}{n_{0}} \theta_{s}\right) \partial_{\left(x^{\prime}, \theta_{p}\right)}^{\alpha_{2}} \partial_{\theta_{s}} \sigma_{s, k}^{n+1}\left(x, \theta_{s}\right)\right|_{0, \gamma, T} d \theta_{s}
$$

where $\left|\alpha_{1}\right|+\left|\alpha_{2}\right|=|\alpha|,\left|\alpha_{1}\right| \geq 1$. Applying $\mathbf{A}$ with $m_{1}=m-\left|\alpha_{1}\right|, m_{2}=\left|\alpha_{1}\right|-1$ together with $\mathbf{B}$, one finds

$$
(3.79) \leq C\left\langle\partial_{\theta_{0}} \chi^{1, n}\right\rangle_{m, T}\left|\sigma_{s, k}^{n+1}\right|_{m, \gamma, T} \leq C K\left|\sigma_{s, k}^{n+1}\right|_{m, \gamma, T}
$$

Normal derivatives are estimated as before yielding (3.76).

4. Uniqueness follows from the $L^{2}$ estimate, the support condition, and the condition that $\chi^{1, n+1}$ has mean zero. It is now straightforward to mimic the finite difference argument of Proposition 2.19 to obtain existence.

The estimate of Proposition 3.10 implies convergence of the iteration scheme by the argument used to prove Proposition 2.21, giving:

Proposition 3.11. (a) Consider the profile equations (3.60), where the perturbing wave $\mathcal{W}^{0} \in \mathbb{H}_{T}^{m+1}, m>\frac{d+3}{2}+1$, has compact $x$-support with

$$
\mathcal{H}\left(x^{\prime}, \theta_{0}\right):=\left.\left[\left(A_{d}(\underline{u})-\sigma A_{0}(\underline{u})\right) \mathcal{W}^{0}\right]_{c}\right|_{x_{d}=0} \in H_{T}^{m} .
$$


For some $0<T_{0} \leq T$ the system has a unique solution $\left(\mathcal{V}^{0}(x, \theta), \chi^{0}\left(x^{\prime}\right), \chi^{1}\left(x^{\prime}, \theta_{0}\right)\right)$ vanishing in $t \leq 0$ with

$$
\left(\mathcal{V}^{0}, \chi^{0}\right) \in \mathbb{H}_{T_{0}}^{m} \times H_{T_{0}}^{m+1}, \quad \partial_{\theta_{0}} \chi^{1} \in H_{T_{0}}^{m},
$$

and such that $\chi^{1} \in H_{T_{0}}^{m}$ has mean zero.

(b)The iterates $\mathcal{V}^{0, n}$ are bounded in $\mathbb{H}_{T_{0}}^{m}$ and converge to $\mathcal{V}^{0}$ in $\mathbb{H}_{T_{0}}^{m-1}$. The iterates $\left(d_{x^{\prime}} \chi^{0, n}, \partial_{\theta_{0}} \chi^{1, n}\right)$ are bounded in $H_{T_{0}}^{m}$ and converge to $\left(d_{x^{\prime}} \chi^{0}, \partial_{\theta_{0}} \chi^{1}\right)$ in $H_{T_{0}}^{m-1}$.

Defining $\mathcal{U}^{0 \pm}$ by (3.61) and writing the corresponding iterates as $\mathcal{U}^{0, n}$ we now have a solution $\left(\mathcal{U}^{0}\left(x, \theta_{0}, \xi_{d}\right), \chi^{0}\left(x^{\prime}\right), \chi^{1}\left(x^{\prime}, \theta_{0}\right)\right)$ of the almost-periodic profile system (3.49). Proposition 3.11(b) and Lemma 2.25 imply:

$$
\text { the iterates } \mathcal{U}^{0, n} \text { are bounded in } \mathcal{E}_{T_{0}}^{m-1} \text { and } \mathcal{U}^{0, n} \rightarrow \mathcal{U}^{0} \text { in } \mathcal{E}_{T_{0}}^{m-2}
$$

Remark 3.12. The condition (3.81) is satisfied provided corner compatibility (3.34) holds for $k_{0}$ large enough.

\subsection{Error analysis}

In this section we justify the leading order expansion of the solution $\left(u_{\epsilon}, \psi_{\epsilon}\right)$ to the perturbed shock problem (3.25). In its simplest form the main result is:

Theorem 3.13. The exact solution $\left(u_{\epsilon}, \psi_{\epsilon}\right)$ of the perturbed shock problem (3.25) satisfies on $\left[0, T_{0}\right] \times \mathbb{R}^{d}$ :

$$
\begin{aligned}
& \lim _{\epsilon \rightarrow 0}\left|\frac{u_{\epsilon}^{ \pm}(x)-\underline{u}^{ \pm}}{\epsilon}-\left(\mathcal{W}^{0 \pm}\left(x, \frac{\phi_{0}\left(x^{\prime}\right)}{\epsilon}, \frac{x_{d}}{\epsilon}\right)+\mathcal{U}^{0 \pm}\left(x, \frac{\phi_{0}\left(x^{\prime}\right)}{\epsilon}, \frac{x_{d}}{\epsilon}\right)\right)\right|_{C^{0}(x)}=0 \\
& \lim _{\epsilon \rightarrow 0}\left|\frac{\left(\psi_{\epsilon}\left(x^{\prime}\right)-\sigma x_{0}\right)}{\epsilon}-\left(\chi^{0}\left(x^{\prime}\right)+\epsilon \chi^{1}\left(x^{\prime}, \frac{\phi_{0}\left(x^{\prime}\right)}{\epsilon}\right)\right)\right|_{C^{1}\left(x^{\prime}\right)}=0
\end{aligned}
$$

where $\mathcal{W}^{0 \pm}$ is the leading profile of the perturbing wave and $\left(\mathcal{U}^{0 \pm}, \chi^{0}, \chi^{1}\right)$ are the profiles constructed in section 3.3.

One can, of course, use (3.61) to rewrite $\mathcal{U}^{0 \pm}\left(x, \frac{\phi_{0}\left(x^{\prime}\right)}{\epsilon}, \frac{x_{d}}{\epsilon}\right)$ in terms of $\underline{v}^{ \pm}, \sigma_{m, k}^{ \pm}$as in (1.24). For $\mathcal{W}^{0 \pm}\left(x, \frac{\phi_{0}\left(x^{\prime}\right)}{\epsilon}, \frac{x_{d}}{\epsilon}\right)$ one can use (3.64). Theorem 3.13 is an immediate corollary of (3.32) and the following result.

Theorem 3.14. Let $M_{0}=2(d+2)+1$ and $s \geq 1+\left[M_{0}+\frac{d+1}{2}\right]$. Choose the perturbing wave $W_{\epsilon}^{ \pm}$as in Theorem 3.6, where $\mathcal{W}^{0}$ in (3.32) is taken such that (3.81) holds with $m=s+1$. Let $\left(U_{\epsilon}\left(x, \theta_{0}\right), \chi_{\epsilon}\left(x^{\prime}, \theta_{0}\right)\right) \in E_{T_{0}}^{s} \times H_{T_{0}}^{s+1}$ be the exact solution to the singular system (3.39) for $0<\epsilon \leq \epsilon_{0}$ given by Theorem 3.6, let $\left(\mathcal{V}^{0}(x, \theta), \chi^{0}\left(x^{\prime}\right), \chi^{1}\left(x^{\prime}, \theta_{0}\right)\right) \in \mathbb{H}_{T_{0}}^{s+1} \times H_{T_{0}}^{s+2} \times H_{T_{0}}^{s+1}$ be the profiles given by Proposition 2.21, and let $\mathcal{U}^{0} \in \mathcal{P}_{T_{0}}^{s}$ be defined by

$$
\mathcal{U}^{0}\left(x, \theta_{0}, \xi_{d}\right)=\mathcal{V}^{0}\left(x, \theta_{0}, \theta_{0}+\underline{\omega}_{1} \xi_{d}, \ldots, \theta_{0}+\underline{\omega}_{M} \xi_{d}\right) .
$$


Here $T_{0}>0$ is the minimum of the existence times for the quasilinear problems (3.39) and (3.60). The family

$$
\mathcal{U}_{\epsilon}^{0}\left(x, \theta_{0}\right):=\mathcal{U}^{0}\left(x, \theta_{0}, \frac{x_{d}}{\epsilon}\right)
$$

is uniformly bounded in $E_{T_{0}}^{s}$ for $0<\epsilon \leq \epsilon_{0}$ and satisfies

$$
\left|U_{\epsilon}-\mathcal{U}_{\epsilon}^{0}\right|_{E_{T_{0}}^{s-1}} \rightarrow 0 \text { as } \epsilon \rightarrow 0 .
$$

Moreover, with $\nabla^{\epsilon}=\partial_{x^{\prime}}+\frac{\beta \partial_{\theta_{0}}}{\epsilon}$, the family $\nabla^{\epsilon}\left(\chi^{0}+\epsilon \chi^{1}\right)$ is bounded in $H_{T_{0}}^{s+1}$ and

$$
\left|\nabla^{\epsilon}\left(\chi_{\epsilon}-\left(\chi^{0}+\epsilon \chi^{1}\right)\right)\right|_{H_{T_{0}}^{s}} \rightarrow 0 \text { as } \epsilon \rightarrow 0
$$

The proof of Theorem 3.14 by simultaneous Picard iteration has much in common with the proof of Theorem 2.22 for the fixed boundary case, but now we must incorporate the free boundary and its profiles into the argument. For easy comparison we now write out the iteration schemes for solving the exact singular shock and shock profile problems:

(a) $\partial_{x_{d}} U_{\epsilon}^{n+1}+\sum_{j=0}^{d-1} \tilde{A}_{j}\left(\underline{u}+\epsilon W_{\epsilon}+\epsilon U_{\epsilon}^{n}, \nabla^{\epsilon}\left(\sigma x_{0}+\epsilon \chi_{\epsilon}^{n}\right)\right)\left(\partial_{x_{j}}+\frac{\beta_{j} \partial_{\theta_{0}}}{\epsilon}\right) U_{\epsilon}^{n+1}=$

$$
-\sum_{j=0}^{d-1}\left(\tilde{A}_{j}\left(\underline{u}+\epsilon W_{\epsilon}+\epsilon U_{\epsilon}^{n}, \nabla^{\epsilon}\left(\sigma x_{0}+\epsilon \chi_{\epsilon}^{n}\right)\right)-\tilde{A}_{j}\left(\underline{u}+\epsilon W_{\epsilon}, \nabla^{\epsilon}\left(\sigma x_{0}\right)\right)\right)\left(\partial_{x_{j}}+\frac{\beta_{j} \partial_{\theta_{0}}}{\epsilon}\right) W_{\epsilon},
$$

(b) $\sum_{j=0}^{d-1}\left(\int_{0}^{1}\left[f_{j}\left(\underline{u}+\epsilon W_{\epsilon}+s \epsilon U_{\epsilon}^{n}\right)\right] d s\right)\left(\partial_{x_{j}}+\frac{\beta_{j} \partial_{\theta_{0}}}{\epsilon}\right) \chi_{\epsilon}^{n+1}-$

$$
\int_{0}^{1}\left[\mathcal{A}_{d}\left(\underline{u}+\epsilon W_{\epsilon}+s \epsilon U_{\epsilon}^{n}, \nabla^{\epsilon}\left(\sigma x_{0}+s \epsilon \chi_{\epsilon}^{n}\right)\right) U_{\epsilon}^{n+1}\right] d s=-\mathcal{G}_{\epsilon}\left(x^{\prime}, \theta_{0}\right)_{c} \text { on } x_{d}=0,
$$

(c) $U_{\epsilon}^{n+1}=0$ and $\chi_{\epsilon}^{n+1}=0$ in $x_{0}<0$,

and 
(3.90)

(a) $\mathbb{E} \mathcal{U}^{0, n+1}=\mathcal{U}^{0, n+1}$

(b) $\mathbb{E}\left(\tilde{L}\left(\partial_{x}\right) \mathcal{U}^{0, n+1}+\mathcal{M}_{1}\left(\mathcal{W}^{0}+\mathcal{U}^{0, n}\right) \partial_{\theta_{0}} \mathcal{U}^{0, n+1}+\mathcal{M}_{2}\left(d_{x^{\prime}} \chi^{0, n}, \partial_{\theta_{0}} \chi^{1, n}\right) \partial_{\theta_{0}} \mathcal{U}^{0, n+1}\right)=$ $-\mathbb{E}\left(\mathcal{M}_{1}\left(\mathcal{U}^{0, n}\right) \partial_{\theta_{0}} \mathcal{W}^{0}+\mathcal{M}_{2}\left(d_{x^{\prime}} \chi^{0, n}, \partial_{\theta_{0}} \chi^{1, n}\right) \partial_{\theta_{0}} \mathcal{W}^{0}\right)$

(c) $G(\beta) \partial_{\theta_{0}} \chi^{0, n+1}:=\sum_{j=0}^{d-1}\left[f_{j}(\underline{u})\right] \beta_{j} \partial_{\theta_{0}} \chi^{0, n+1}=0$

(d) $G(\beta) \partial_{\theta_{0}} \chi^{1, n+1}+\sum_{j=0}^{d-1}\left[f_{j}(\underline{u})\right] \partial_{x_{j}} \chi^{0, n+1}+\left[\left(\sigma A_{0}(\underline{u})-A_{d}(\underline{u})\right) \mathcal{U}^{0, n+1}\right]=\left[\left(A_{d}(\underline{u})-\sigma A_{0}(\underline{u})\right) \mathcal{W}^{0}\right]_{c}$

(e) $\mathcal{U}^{0, n+1}=0, \chi^{0, n+1}=0$, and $\chi^{1, n+1}=0$ in $x_{0} \leq 0$.

The iteration schemes are initialized by taking $U_{\epsilon}^{0}=0, \chi_{\epsilon}^{0}=0, \mathcal{U}^{0,0}=0, \chi^{0,0}=0$, and $\chi^{1,0}=0$. The induction step in the simultaneous Picard iteration will be completed by applying the estimate in the next Proposition.

Proposition 3.15 ([23], Cor. 9.1). (a) Let $s \geq\left[M_{0}+\frac{d+1}{2}\right]$ and consider the problem (3.89), where $W_{\epsilon}$ is chosen as in Theorem 3.6 and where the right sides of equations (3.89)(a) and (b) are replaced by $F\left(x, \theta_{0}\right)$ and $G\left(x^{\prime}, \theta_{0}\right)$ respectively. We assume $G \in H_{T}^{s+1}$ with supp $G \subset\{t \leq 0\}$ and $F \in \mathbb{E}_{T}^{s}$ with $\operatorname{supp} F \subset\left\{t \geq 0,0 \leq x_{d} \leq E\right\}$. Suppose $\chi_{\epsilon}^{n} \in H_{T}^{s+1}$ has compact $x^{\prime}$-support and that $\nabla^{\epsilon} \chi_{\epsilon}^{n} \in H_{T}^{s+1}$. Suppose $U_{\epsilon}^{n} \in E_{T}^{s}$ has compact $x$-support and that for some $K>0, \epsilon_{1}>0$ we have

$$
\left|U_{\epsilon}^{n}\right|_{E_{T}^{s}}+\left|\epsilon \partial_{x_{d}} U_{\epsilon}^{n}\right|_{L^{\infty}}+\left\langle\nabla^{\epsilon} \chi_{\epsilon}^{n}\right\rangle_{s+1, T} \leq K \text { for } \epsilon \in\left(0, \epsilon_{1}\right] .
$$

Then there exist constants $T_{0}(K)$ and $\epsilon_{0}(K) \leq \epsilon_{1}$ such that for $0<\epsilon \leq \epsilon_{0}$ and $T \leq T_{0}$ we have

$$
\left|U_{\epsilon}^{n+1}\right|_{E_{T}^{s}}+\sqrt{T}\left\langle U_{\epsilon}^{n+1}\right\rangle_{s+1, T}+\sqrt{T}\left\langle\nabla^{\epsilon} \chi_{\epsilon}^{n+1}\right\rangle_{s+1, T} \leq C(K, E) \sqrt{T}\left(|F|_{E_{T}^{s}}+\langle G\rangle_{s+1, T}\right) .
$$

(b) The estimate (3.92) continues to hold if one assumes for $W_{\epsilon}$ the same properties that are assumed for $U_{\epsilon}^{n}$.

Proof of Theorem 3.14. 1. Assertions (3.87) and (3.88) of Theorem 3.14 are a consequence of the following three statements:

(a) The families $\left\{U_{\epsilon}, U_{\epsilon}^{n}\right\}_{\epsilon \in\left(0, \epsilon_{0}\right], n \in\{0,1,2, \ldots\}}$ and $\left\{\nabla^{\epsilon} \chi_{\epsilon}, \nabla^{\epsilon} \chi_{\epsilon}^{n}\right\}_{\epsilon \in\left(0, \epsilon_{0}\right], n \in\{0,1,2, \ldots\}}$ are bounded in $E_{T_{0}}^{s}$ and $H_{T_{0}}^{s+1}$ respectively and

$$
\left|U_{\epsilon}^{n}-U_{\epsilon}\right|_{E_{T_{0}}^{s-1}} \rightarrow 0 \text { and }\left\langle\nabla^{\epsilon}\left(\chi_{\epsilon}^{n}-\chi_{\epsilon}\right)\right\rangle_{s, T_{0}} \rightarrow 0 \text { as } n \rightarrow \infty
$$

uniformly with respect to $\epsilon \in\left(0, \epsilon_{0}\right]$. 
(b) The families $\left\{\mathcal{U}_{\epsilon}^{0}, \mathcal{U}_{\epsilon}^{0, n}\right\}_{\epsilon \in\left(0, \epsilon_{0}\right], n \in\{0,1,2, \ldots\}}$ and $\left\{\nabla^{\epsilon}\left(\chi^{0}+\epsilon \chi^{1}\right), \nabla^{\epsilon}\left(\chi^{0, n}+\epsilon \chi^{1, n}\right)\right\}_{\epsilon \in\left(0, \epsilon_{0}\right], n \in\{0,1,2, \ldots\}}$ are bounded in $E_{T_{0}}^{s}$ and $H_{T_{0}}^{s+1}$ respectively and

$$
\left|\mathcal{U}_{\epsilon}^{0, n}-\mathcal{U}_{\epsilon}^{0}\right|_{E_{T_{0}}^{s-1}} \rightarrow 0 \text { and }\left\langle\nabla^{\epsilon}\left(\chi^{0, n}+\epsilon \chi^{1, n}\right)-\nabla^{\epsilon}\left(\chi^{0}+\epsilon \chi^{1}\right)\right\rangle_{s, T_{0}} \rightarrow 0 \text { as } n \rightarrow \infty
$$

uniformly with respect to $\epsilon \in\left(0, \epsilon_{0}\right]$.

(c) For each $n$

$$
\begin{aligned}
& \lim _{\epsilon \rightarrow 0}\left|U_{\epsilon}^{n}-\mathcal{U}_{\epsilon}^{0, n}\right|_{E_{T_{0}}^{s-1}}=0 \text { and } \\
& \lim _{\epsilon \rightarrow 0}\left\langle\nabla^{\epsilon}\left(\chi_{\epsilon}^{n}-\left(\chi^{0, n}+\epsilon \chi^{1, n}\right)\right)\right\rangle_{s, T_{0}}=0 .
\end{aligned}
$$

Statement (a) is proved in [23], Theorem 9.4, by using the estimate of Proposition 3.15 to show convergence of the iteration scheme (3.89). Statement (b) follows directly from Proposition 3.11, (3.83), and (2.111). We now assume that (c), which holds trivially for $n=0$, holds for a fixed $n$, and then show it holds for $n+1$.

2. Fix $\delta>0$ and choose trigonometric polynomials $\mathcal{U}_{p}^{0, n}, \mathcal{U}_{p}^{0, n+1}$ with $H^{\infty}(x)$ coefficients such that (2.115) (reinterpreted in the shock context) holds. Similarly choose trigonometric polynomials with $H^{\infty}\left(x^{\prime}\right)$ coefficients such that

$$
\left\langle\left(d_{x^{\prime}} \chi_{p}^{0, n}, \partial_{\theta_{0}} \chi_{p}^{1, n}\right)-\left(d_{x^{\prime}} \chi^{0, n}, \partial_{\theta_{0}} \chi^{1, n}\right)\right\rangle_{s+1, T_{0}}<\delta .
$$

Note that $\chi_{p}^{0, n}$ and $\chi_{p}^{0, n+1}$ are just $H^{\infty}\left(x^{\prime}\right)$ functions. Finally, choose $\mathcal{W}_{p}^{0}$ such that

$$
\left|\mathcal{W}^{0}-\mathcal{W}_{p}^{0}\right|_{\mathcal{E}_{T_{0}}^{s}}<\delta
$$

We choose all trigonometric polynomials to have compact support in their spatial arguments $x$ or $x^{\prime}$

3. Let us set

$$
\begin{gathered}
\mathcal{P}_{\epsilon}^{n} v:=\partial_{x_{d}} v+\sum_{j=0}^{d-1} \tilde{A}_{j}\left(\underline{u}+\epsilon \mathcal{W}_{p, \epsilon}^{0}+\epsilon \mathcal{U}_{p, \epsilon}^{0, n}, \nabla^{\epsilon}\left(\sigma x_{0}+\epsilon\left(\chi_{p}^{0, n}+\epsilon \chi_{p}^{1, n}\right)\right)\right)\left(\partial_{x_{j}}+\frac{\beta_{j} \partial_{\theta_{0}}}{\epsilon}\right) v \\
\mathcal{B}_{\epsilon}^{n}\left(v, \nabla^{\epsilon} \phi\right):=\sum_{j=0}^{d-1}\left(\int_{0}^{1}\left[f_{j}\left(\underline{u}+\epsilon \mathcal{W}_{p, \epsilon}^{0}+s \epsilon \mathcal{U}_{p, \epsilon}^{0, n}\right)\right] d s\right)\left(\partial_{x_{j}}+\frac{\beta_{j} \partial_{\theta_{0}}}{\epsilon}\right) \phi- \\
\int_{0}^{1}\left[\mathcal{A}_{d}\left(\underline{u}+\epsilon \mathcal{W}_{p, \epsilon}^{0}+s \epsilon \mathcal{U}_{p, \epsilon}^{0, n}, \nabla^{\epsilon}\left(\sigma x_{0}+s \epsilon\left(\chi_{p}^{0, n}+\epsilon \chi_{p}^{1, n}\right)\right)\right) v\right] d s
\end{gathered}
$$

We will construct a trigonometric polynomial corrector $\mathcal{U}_{p}^{1}$ such that

(a) $\left|\mathcal{P}_{\epsilon}^{n}\left(U_{\epsilon}^{n+1}-\left(\mathcal{U}_{p, \epsilon}^{0, n+1}+\epsilon \mathcal{U}_{p, \epsilon}^{1}\right)\right)\right|_{E_{T_{0}}^{s-1}} \leq C \delta+c(\epsilon)+K(\delta) \epsilon$
(b) $\left\langle\mathcal{B}_{\epsilon}^{n}\left(U_{\epsilon}^{n+1}-\left(\mathcal{U}_{p, \epsilon}^{0, n+1}+\epsilon \mathcal{U}_{p, \epsilon}^{1}\right), \nabla^{\epsilon}\left(\chi_{\epsilon}^{n+1}-\left(\chi^{0, n+1}+\epsilon \chi^{1, n+1}\right)\right)\right)\right\rangle_{s, T_{0}} \leq C \delta+c(\epsilon)+K(\delta) \epsilon$. 
An application of Proposition 3.15 yields

$(3.100)$

$\left|U_{\epsilon}^{n+1}-\left(\mathcal{U}_{p, \epsilon}^{0, n+1}+\epsilon \mathcal{U}_{p, \epsilon}^{1}\right)\right|_{E_{T_{0}}^{s-1}}+\left\langle\nabla^{\epsilon}\left(\chi_{\epsilon}^{n+1}-\left(\chi^{0, n+1}+\epsilon \chi^{1, n+1}\right)\right)\right\rangle_{s, T_{0}} \leq C \delta+c(\epsilon)+K(\delta) \epsilon$,

where $c(\epsilon) \rightarrow 0$ as $\epsilon \rightarrow 0$ and $C$ is independent of $\delta$ and $\epsilon$. The estimate (3.100) implies (3.95) for $n+1$, since $\left|\epsilon \mathcal{U}_{p, \epsilon}^{1}\right|_{E_{T_{0}}^{s-1}} \leq K(\delta) \epsilon$ and $\left|\mathcal{U}_{\epsilon}^{0, n+1}-\mathcal{U}_{p, \epsilon}^{0, n+1}\right|_{E_{T_{0}}^{s-1}} \leq C \delta$.

4. Let us denote the right side of (3.89)(a) by $F_{\epsilon}\left(W_{\epsilon}, U_{\epsilon}^{n}, \chi_{\epsilon}^{n}\right)$ and set

$$
\mathcal{F}\left(\mathcal{W}_{p}^{0}, \mathcal{U}_{p}^{0, n}, \chi_{p}^{0, n}, \chi_{p}^{1, n}\right):=\mathcal{M}_{1}\left(\mathcal{U}_{p}^{0, n}\right) \partial_{\theta_{0}} \mathcal{W}_{p}^{0}+\mathcal{M}_{2}\left(d_{x^{\prime}} \chi_{p}^{0, n}, \partial_{\theta_{0}} \chi_{p}^{1, n}\right) \partial_{\theta_{0}} \mathcal{W}_{p}^{0}
$$

After Taylor expanding $\tilde{A}_{j}$ about $\left(\underline{u}, d_{x^{\prime}}\left(\sigma x_{0}\right)\right)$ and using (3.32), the approximation estimates of step 2, and the induction assumption, we find (parallel to (2.118)) that

$$
\left|F_{\epsilon}\left(W_{\epsilon}, U_{\epsilon}^{n}, \chi_{\epsilon}^{n}\right)-\mathcal{F}\left(\mathcal{W}_{p}^{0}, \mathcal{U}_{p}^{0, n}, \chi_{p}^{0, n}, \chi_{p}^{1, n}\right)_{\epsilon}\right|_{E_{T_{0}}^{s-1}} \leq C \delta+c(\epsilon) .
$$

For example, a typical estimate leading to (3.102) is

$$
\begin{aligned}
&\left(\partial_{\omega} \tilde{A}_{j}\left(\underline{u}, d_{x^{\prime}}\left(\sigma x_{0}\right)\right) \cdot \epsilon \nabla^{\epsilon} \chi_{\epsilon}^{n}\right)\left(\partial_{x_{j}}+\frac{\beta_{j} \partial_{\theta_{0}}}{\epsilon}\right) W_{\epsilon}- \\
&\left(\partial_{\omega} \tilde{A}_{j}\left(\underline{u}, d_{x^{\prime}}\left(\sigma x_{0}\right)\right) \cdot\left(d_{x^{\prime}} \chi^{0, n}+\beta \partial_{\theta_{0}} \chi^{1, n}\right)\right) \beta_{j} \partial_{\theta_{0}} \mathcal{W}_{\epsilon}^{0}=c(\epsilon) \text { in } E_{T_{0}}^{s-1},
\end{aligned}
$$

where the term being subtracted is the $j$-th term in the sum defining $\mathcal{M}_{2}\left(d_{x^{\prime}} \chi^{0, n}, \partial_{\theta_{0}} \chi^{1, n}\right) \partial_{\theta_{0}} \mathcal{W}_{\epsilon}^{0}$. Replacing the arguments in the subtracted term by their trigonometric polynomial approximations introduces another error of size $C \delta$ in $E_{T_{0}}^{s-1}$.

5. Define

$$
\mathcal{G}_{p}=\tilde{L}\left(\partial_{x}\right) \mathcal{U}_{p}^{0, n+1}+\mathcal{M}_{1}\left(\mathcal{W}_{p}^{0}+\mathcal{U}_{p}^{0, n}\right) \partial_{\theta_{0}} \mathcal{U}_{p}^{0, n+1}+\mathcal{M}_{2}\left(d_{x^{\prime}} \chi_{p}^{0, n}, \partial_{\theta_{0}} \chi_{p}^{1, n}\right) \partial_{\theta_{0}} \mathcal{U}_{p}^{0, n+1}
$$

As in step 5 of the proof of Theorem 2.22 we use (3.90)(b) to show

$$
\left|\mathbb{E} \mathcal{G}_{p}-\mathbb{E} \mathcal{F}\left(\mathcal{W}_{p}^{0}, \mathcal{U}_{p}^{0, n}, \chi_{p}^{0, n}, \chi_{p}^{1, n}\right)\right|_{\mathcal{E}_{T_{0}}^{s-1}}=O(\delta) .
$$

6. Define the following approximation to the operator $\mathcal{P}_{\epsilon}^{n}$ appearing in (3.99)(a):

$$
\mathbb{L}_{0}=\tilde{L}\left(\partial_{x}\right)+\frac{1}{\epsilon} \tilde{L}\left(d \phi_{0}\right) \partial_{\theta_{0}}+\mathcal{M}_{1}\left(\mathcal{W}_{p, \epsilon}^{0}+\mathcal{U}_{p, \epsilon}^{0, n}\right) \partial_{\theta_{0}}+\mathcal{M}_{2}\left(d_{x^{\prime}} \chi_{p}^{0, n}, \partial_{\theta_{0}} \chi_{p}^{1, n}\right) \partial_{\theta_{0}} .
$$

Using (3.89)(a) and estimates similar to (3.103) and (2.125), we find (parallel to (2.124))

$$
\left|\mathbb{L}_{0} U_{\epsilon}^{n+1}-F_{\epsilon}\left(W_{\epsilon}, U_{\epsilon}^{n}, \chi_{\epsilon}^{n}\right)\right|_{E_{T_{0}}^{s-1}} \leq C \delta+c(\epsilon) .
$$

7. Construction of the corrector. As in (2.128) we construct a trigonometric polynomial $\mathcal{U}_{p}^{1}$ such that

$$
\tilde{\mathcal{L}}\left(\partial_{\theta_{0}}, \partial_{\xi_{d}}\right) \mathcal{U}_{p}^{1}=-(I-\mathbb{E})\left(\mathcal{G}_{p}-\mathbb{E} \mathcal{F}\left(\mathcal{W}_{p}^{0}, \mathcal{U}_{p}^{0, n}, \chi_{p}^{0, n}, \chi_{p}^{1, n}\right)\right) .
$$


Consequently,

$$
\left|\mathbb{L}_{0}\left(\mathcal{U}_{p, \epsilon}^{0, n+1}+\epsilon \mathcal{U}_{p, \epsilon}^{1}\right)-\mathcal{F}\left(\mathcal{W}_{p}^{0}, \mathcal{U}_{p}^{0, n}, \chi_{p}^{0, n}, \chi_{p}^{1, n}\right)_{\epsilon}\right|_{E_{T_{0}}^{s-1}} \leq C \delta+K(\delta) \epsilon
$$

by the argument that gave (2.129). From (3.102), (3.107), and (3.109) we obtain

$$
\left|\mathbb{L}_{0}\left(U_{\epsilon}^{n+1}-\left(\mathcal{U}_{p, \epsilon}^{0, n+1}+\epsilon \mathcal{U}_{p, \epsilon}^{1}\right)\right)\right|_{E_{T_{0}}^{s-1}} \leq C \delta+c(\epsilon)+K(\delta) \epsilon .
$$

By Taylor expanding the coefficients $\tilde{A}_{j}$ in $\mathcal{P}_{\epsilon}^{n}$ about $\left(\underline{u}, d_{x^{\prime}}\left(\sigma x_{0}\right)\right)$ and doing estimates similar to (3.103) and (2.125), we deduce (3.99)(a) directly from (3.110).

8. Boundary terms. We compare the terms in the exact boundary equation (3.89)(b) to the corresponding terms in the profile boundary equation $(3.90)(\mathrm{d})$. By the induction assumption we have for $j=0, \ldots, d-1$ :

$\left\langle\left[f_{j}\left(\underline{u}+\epsilon W_{\epsilon}+s \epsilon U_{\epsilon}^{n}\right)\right]\left(\partial_{x_{j}}+\frac{\beta_{j} \partial_{\theta_{0}}}{\epsilon}\right) \chi_{\epsilon}^{n+1}-\left[f_{j}(\underline{u})\right]\left(\partial_{x_{j}} \chi^{0, n+1}+\beta_{j} \partial_{\theta_{0}} \chi^{1, n+1}\right)\right\rangle_{s, T_{0}} \leq c(\epsilon)$.

Since $\mathcal{A}_{d}\left(v, d_{x^{\prime}} \phi\right)=A_{d}(v)-\sum_{j=0}^{d-1} \phi_{x_{j}} A_{j}(v)$ and $\nabla^{\epsilon} \chi_{\epsilon}^{n}$ is bounded in $H_{T_{0}}^{s}$ (in fact, in $\left.H_{T_{0}}^{s+1}\right)$, we find

$$
\left\langle\left[\mathcal{A}_{d}\left(\underline{u}+\epsilon W_{\epsilon}+s \epsilon U_{\epsilon}^{n}, \nabla^{\epsilon}\left(\sigma x_{0}+s \epsilon \chi_{\epsilon}^{n}\right)\right) U_{\epsilon}^{n+1}\right]-\left[\left(A_{d}(\underline{u})-\sigma A_{0}(\underline{u})\right) \mathcal{U}_{\epsilon}^{0, n+1}\right]\right\rangle_{s, T_{0}} \leq O(\epsilon) .
$$

Moreover, since $\sigma\left[f_{0}(\underline{u})\right]=\left[f_{d}(\underline{u})\right]$ and $\mathcal{G}_{\epsilon}\left(x^{\prime}, \theta_{0}\right)=\frac{1}{\epsilon}\left(\sigma\left[f_{0}\left(\underline{u}+\epsilon W_{\epsilon}\right)\right]-\left[f_{d}\left(\underline{u}+\epsilon W_{\epsilon}\right)\right]\right)$, it follows that

$$
\left\langle\mathcal{G}_{\epsilon}\left(x^{\prime}, \theta_{0}\right)_{c}-\left[\left(\sigma A_{0}(\underline{u})-A_{d}(\underline{u})\right) \mathcal{W}^{0}\right]_{c}\right\rangle_{s, T_{0}}=O(\epsilon) .
$$

Taking into account the $O(\epsilon)+O(\delta)$ errors introduced by, for example, replacing $W_{\epsilon}$ by $\mathcal{W}_{p, \epsilon}^{0}$, we see that the above three estimates imply

$$
\left\langle\mathcal{B}_{\epsilon}^{n}\left(U_{\epsilon}^{n+1}-\mathcal{U}_{p, \epsilon}^{0, n+1}, \nabla^{\epsilon}\left(\chi_{\epsilon}^{n+1}-\left(\chi^{0, n+1}+\epsilon \chi^{1, n+1}\right)\right)\right)\right\rangle_{s, T_{0}} \leq C \delta+c(\epsilon) .
$$

Finally, since $\left\langle\epsilon \mathcal{U}_{p, \epsilon}^{1}\right\rangle_{s, T_{0}} \leq K(\delta) \epsilon$, the estimate (3.99)(b) follows. This completes the proof of Theorem 3.14.

\section{References}

$[1]$

[2] S. Benzoni-Gavage, D. Serre. Multidimensional hyperbolic partial differential equations. Oxford Mathematical Monographs. Oxford University Press, 2007. 
[3] J. Chazaran, J. Piriou. Introduction to the Theory of Linear Partial Differential Equations. North Holland, 1982.

[4] A. Corli. Weakly non-linear geometric optics for hyperbolic systems of conservation laws with shock waves. Asymptotic Analysis, 10(2):117-172, 1995.

[5] J.-F. Coulombel and O. Gues. Geometric optics expansions with amplification for hyperbolic boundary value problems: linear problems. to appear in Annales de l'Institut Fourier.

[6] Métivier G. Williams M. Guès, O. and K. Zumbrun. Existence and stability of noncharacteristic boundary layers for the compressible navier-stokes and viscous mhd equations.

[7] O. Guès. Développement asymptotique de solutions exactes de systèmes hyperboliques quasilinéaires. Asymptotic Anal., 6(3):241-269, 1993.

[8] J.-L. Joly, G. Métivier, and J. Rauch. Coherent and focusing multidimensional nonlinear geometric optics. Ann. Sci. École Norm. Sup. (4), 28(1):51-113, 1995.

[9] H.-O. Kreiss. Initial boundary value problems for hyperbolic systems. Comm. Pure Appl. Math., 23:277-298, 1970.

[10] P. D. Lax. Asymptotic solutions of oscillatory initial value problems. Duke Math. J., 24:627-646, 1957.

[11] V. Lescarret. Wave transmission in dispersive media. Math. Models Methods Appl. Sci., 17(4):485-535, 2007.

[12] A. Majda. The existence of multidimensional shock fronts. Mem. Amer. Math. Soc., $281,1983$.

[13] A. Majda. The stability of multidimensional shock fronts. Mem. Amer. Math. Soc., $275,1983$.

[14] A. Majda and M. Artola. Nonlinear geometric optics for hyperbolic mixed problems. In Analyse mathématique et applications, pages 319-356. Gauthier-Villars, 1988.

[15] A. Marcou. Rigorous weakly nonlinear geometric optics for surface waves. to appear in Asymptotic Analysis.

[16] G. Métivier. Problemes mixtes nonlineaires et stabilite des chocs multidimensionnels. Sem. Bourbaki, 671:37-53, 1986.

[17] G. Métivier. The block structure condition for symmetric hyperbolic systems. Bull. London Math. Soc., 32(6):689-702, 2000. 
[18] G. Métivier. Stability of multidimensional shocks. volume 47 of Advances in the Theory of Shock Waves. Birkhäuser, Boston, 2001.

[19] Y. Wang. Nonlinear geometric optics for shock waves. ii. system case. Z. Anal. Anwendungen, 16:857-918, 1997.

[20] M. Williams. Nonlinear geometric optics for hyperbolic boundary problems. Comm. Partial Differential Equations, 21(11-12):1829-1895, 1996.

[21] M. Williams. Highly oscillatory multidimensional shocks. Comm. Pure Appl. Math., 52:129-192, 1999.

[22] M. Williams. Boundary layers and glancing blow-up in nonlinear geometric optics. Ann. Sci. École Norm. Sup. (4), 33(3):383-432, 2000.

[23] M. Williams. Singular pseudodifferential operators, symmetrizers, and oscillatory multidimensional shocks. J. Functional Analysis, 191:132-209, 2002. 